University of Louisville

ThinkIR: The University of Louisville's Institutional Repository

Electronic Theses and Dissertations

$12-2012$

\title{
A study of the total coloring of graphs.
}

Maxfield Edwin Leidner

University of Louisville

Follow this and additional works at: https://ir.library.louisville.edu/etd

\section{Recommended Citation}

Leidner, Maxfield Edwin, "A study of the total coloring of graphs." (2012). Electronic Theses and Dissertations. Paper 815.

https://doi.org/10.18297/etd/815

This Doctoral Dissertation is brought to you for free and open access by ThinkIR: The University of Louisville's Institutional Repository. It has been accepted for inclusion in Electronic Theses and Dissertations by an authorized administrator of ThinkIR: The University of Louisville's Institutional Repository. This title appears here courtesy of the author, who has retained all other copyrights. For more information, please contact thinkir@louisville.edu. 


\title{
A STUDY OF THE TOTAL COLORING OF GRAPHS
}

\author{
By \\ Maxfield Edwin Leidner \\ B.S., University of Louisville, 2006 \\ M.A., University of Louisville, 2010 \\ A Dissertation \\ Submitted to the Faculty of the \\ College of Arts and Sciences of the University of Louisville \\ in Partial Fulfillment of the Requirements \\ for the Degree of
}

Doctor of Philosophy

Department of Mathematics

University of Louisville

Louisville, KY

December 2012 


\title{
A STUDY OF THE TOTAL COLORING OF GRAPHS
}

\author{
Submitted by \\ Maxfield Edwin Leidner
}

A Dissertation Approved on

November 20, 2012

(Date)

by the Following Reading and Examination Committee:

\begin{tabular}{c}
\hline Grzegorz Kubicki, Dissertation Director \\
\hline Ewa Kubicka \\
\hline André E. Kézdy \\
\hline David Wildstrom \\
\hline Rammohan K. Ragade
\end{tabular}

\author{
Rammohan K. Ragade
}




\section{ACKNOWLEDGEMENTS}

I have three people to thank for getting me interested in graph theory. The first is my grandfather, Edwin Leidner, who, in my childhood, told me to solve the utility graph problem, and promised me it could be done. (The problem was to draw lines connecting each of three houses to each of three utility companies so that no two lines cross. This is impossible to do on a flat surface.) The second is my thesis advisor, Dr. Grzegorz Kubicki, who taught a very memorable discrete mathematics course in 2002. Taking that course gave me confidence that I had some mathematical ability, gave me a direction, and convinced me that teaching math could be very cool. The third was my graduate advisor and instructor in combinatorics and graph theory, Dr. André Kézdy. If it weren't for his course and his motivational attitude about problem solving, which further cultivated my love of discrete mathematics, I might still be trying to decide what to write about.

For giving me a place to find myself, as well as years of experience and life support through teaching, I offer my gratitude to the University of Louisville Department of Mathematics. I thank my parents, Al and Lil, for their constant love, support, encouragement, and much more. Most importantly, I thank my loving wife Chenchen, for giving me all this plus an affirmation of life, happy thoughts about the future, and the idea that I probably shouldn't be a student forever. 


\section{ABSTRACT \\ A STUDY OF THE TOTAL COLORING OF GRAPHS}

\section{Maxfield Edwin Leidner}

December 13, 2012

The area of total coloring is a more recent and less studied area than vertex and edge coloring, but recently, some attention has been given to the Total Coloring Conjecture, which states that each graph's total chromatic number $\chi^{T}$ is no greater than its maximum degree plus two. In this dissertation, it is proved that the conjecture is satisfied by those planar graphs in which no vertex of degree 5 or 6 lies on more than three 3-cycles. The total independence number $\alpha^{T}$ is found for some families of graphs, and a relationship between that parameter and the size of a graph's minimum maximal matching is discussed. For colorings with natural numbers, the total chromatic sum $\Sigma^{T}$ is introduced, as is total strength $\sigma^{T}$ of a graph. Tools are developed for proving that a total coloring has minimum sum, and this sum is found for some graphs including paths, cycles, complete graphs, complete bipartite graphs, full binary trees, and some hypercubes. A family of graphs is found for which no optimal total coloring maximizes the smallest color class. Lastly, the relationship between a graph's total chromatic number and its total strength is explored, and some graphs are found that require more than their total chromatic number of colors to obtain a minimum sum. 


\section{TABLE OF CONTENTS}

\section{CHAPTER}

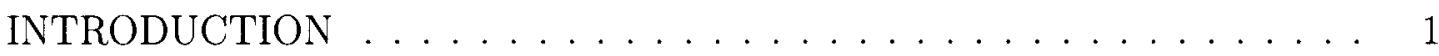

0.1 Traditional Notation and Definitions . . . . . . . . . . . 1

0.2 New Notation and Definitions . . . . . . . . . . . 3

0.3 Application Areas of Total Coloring . . . . . . . . . . . . . . 4

1. PLANAR GRAPHS AND THE TOTAL COLORING CONJECTURE 7

1.1 History of the Total Chromatic Number . . . . . . . . . . . 7

1.2 A Larger Family of $\Delta=6$ Graphs that Satisfy the Conjecture . 9

1.3 Supporting Lemmas for Theorem 1 . . . . . . . . . . . . . . . . 10

1.4 Discharging . . . . . . . . . . . . . . . . . 21

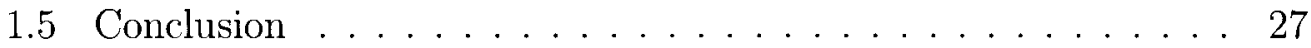

1.6 The Problem with Triangles . . . . . . . . . . . . . . . 27

2. TOTAL INDEPENDENT SETS AND THE TOTAL INDEPENDENCE

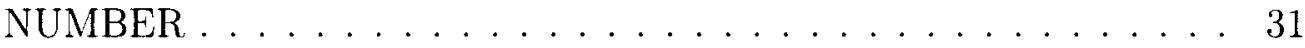

2.1 Independence for all Elements . . . . . . . . . . . . . . . 31

2.2 Total Independence Number of Other Common Graphs . . . . . 34

2.3 Total Independence Number of Full Binary Trees . . . . . . . . . 38

2.4 Total Independence and Edge Domination . . . . . . . . . . . . 42

2.5 Total Independence Number of Hypercubes . . . . . . . . . . . . . 45

3. TOTAL CHROMATIC SUM ................. 50

3.1 History of the Chromatic Sum ... . . . . . . . . 50

3.2 Total Colorings with Optimal Sum . . . . . . . . . . . 53 


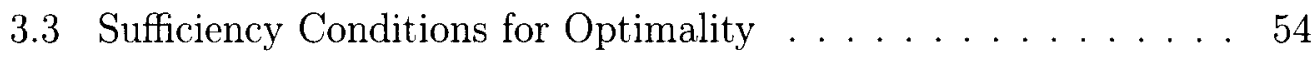

3.4 Total Chromatic Sum of Some Common Graphs . . . . . . . 60

3.5 Total Chromatic Sum of Some Hypercubes . . . . . . . . 66

3.6 Total Chromatic Sum of Full Binary Trees . . . . . . . . 70

3.7 When No Optimal Total Coloring Maximizes $\phi_{1} \ldots \ldots$. . 83

4. THE T-STRENGTH OF GRAPHS . . . . . . . . . . . 84

4.1 Total Strength vs. Total Chromatic Number . . . . . . . . . 84

4.2 Trees of Maximum Distance Four or Less . . . . . . . . . . 84

4.3 The Smallest $T$-Strong Graph $\ldots \ldots \ldots \ldots \ldots$

4.4 Infinite Families of $T$-Strong Graphs . . . . . . . . . . . 92

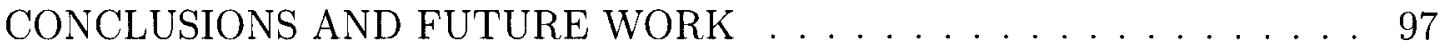

REFERENCES . . . . . . . . . . . . . . . . . . 98

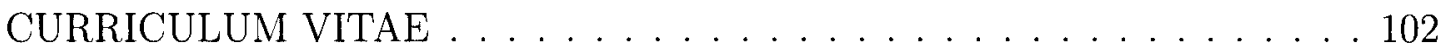




\section{LIST OF FIGURES}

FIGURE 1.1. A total-7-coloring of the graph $I_{1} \ldots \ldots \ldots \ldots$

FIGURE 1.2. A (3,6,6)-triangle, a $(4,4,6)$-triangle, and a $\left(4,5^{-}, 5^{-}\right)$-triangle. $\ldots \ldots 12$

FIGURE 1.3. A 4-face with two 3-vertices, a 5-face with two 3-vertices, and a 6-face with three 3 -vertices. . . . . . . . . . . . . . . 14

FIGURE 1.4. A pair of $(4,5,6)$-triangles that share a common $(5,6)$-edge. . . . . . . 16

FIGURE 1.5. A $(4,5,6)$-triangle sharing an edge with a $\left(3,6,5^{-}, 6\right)$-face. On the left, the $(5,6)$-edge is shared; on the right, the $(4,6)$-edge is shared. . . . 19

FIGURE 1.6. A visual description of the Discharging Rules. . . . . . . . . . . . . 22

FIGURE 1.7. Two configurations in which a 6 -vertex has three incident triangles and one special neighbor. . . . . . . . . . . . . . . . . . 25

FIGURE 1.8. K, a minimum counterexample to Conjecture 1 (or 2), might satisfy the conditions for that Conjecture and still contain a configuration to one shown above. However, this could imply that a subgraph of $K$ might not satisfy the same conditions. $\ldots \ldots \ldots \ldots \ldots$

FIGURE 2.1. Three graphs, all paths on seven vertices, in which a maximum independent set, a maximum matching, and a maximum total independent set are demonstrated. . . . . . . . . . . . . . 32

FIGURE 2.2. Choice of a maximum total independent set in $C_{n}$, depending on $n(\bmod$

$3)$.

FIGURE 2.3. On the left, the 3-cube $Q_{3}$. On the right, the 4-cube $Q_{4}$. In each, a maximum total independent set is labeled in red. 
FIGURE 3.1. A graph shown twice, with two different colorings. The coloring on the left has weight 11 , and the coloring on the right has weight $9 . \ldots 50$

FIGURE 3.2. A graph shown twice, with different colorings. The coloring on the left has weight 12 , and uses only $\chi=2$ colors. The coloring on the right has weight $\Sigma=11$, but uses 3 colors. . . . . . . . . . . . 52

FIGURE 3.3. Giaro's example of a graph with $\Delta=5, \chi^{\prime}=5$, and $\sigma^{\prime} \geq 6 . \ldots 53$

FIGURE 3.4. A demonstration of $\phi$ on paths of three modularities. . . . . . . . . 60

FIGURE 3.5. A demonstration of $\phi$, as described below, on cycles of three modularities. 61

FIGURE 3.6. The total-4-coloring of a 3-cube subgraph of $Q_{n}$, given in step 1 of the algorithm. $($ Red $=1$, Blue $=2$, Green $=3$, and Purple $=4.) \ldots 68$

FIGURE 3.7. A total-3-coloring of $B_{1}$ with sum $9 . \ldots \ldots \ldots$. . . . . . . 71

FIGURE 3.8. A total-4-coloring of $B_{2}$ with sum $27 \ldots \ldots \ldots \ldots \ldots$

FIGURE 3.9. A total-4-coloring of $B_{3}$ with sum $61 \ldots \ldots \ldots$. . . . . . . 73

FIGURE 3.10A total-4-coloring of $B_{4}$ with sum $131 \ldots \ldots \ldots \ldots$

FIGURE 4.1. An example of a tree with maximum distance less than $5 . \quad \ldots \ldots$. . . 85

FIGURE 4.2 . The grid graph $P_{2} \times P_{3}$ total-colored in two ways. $\ldots \ldots \ldots \ldots$

FIGURE 4.3. Four $T$-strong graphs. $\ldots \ldots \ldots \ldots \ldots$ 


\section{LIST OF TABLES}

TABLE 2.1. A comparison of some results for the hypercubes $Q_{3}$ through $Q_{7}$. . . . 48

TABLE 3.1. A table of values and lower bounds for $W_{1}$ using a graph $B_{1}$ having root vertex $v$ and the edges $v u$ and $v w . \ldots \ldots \ldots . \ldots \ldots 71$

TABLE 3.2. A table of values and lower bounds for $W_{2}$ using a graph $B_{2}$ having root vertex $v$ and the edges $v u$ and $v w . \ldots \ldots \ldots \ldots \ldots$. . . . . . . . 72

TABLE 3.3. A table of values and lower bounds for $W_{3}$ using a graph $B_{3}$ having root vertex $v$ and the edges $v u$ and $v w \ldots \ldots \ldots \ldots \ldots$

TABLE 3.4. A table of values and lower bounds for $W_{k}$, with $k \geq 4$, using a graph $B_{k}$ having root vertex $v$ and the edges $v u$ and $v w \ldots \ldots \ldots . \ldots 75$

TABLE 3.5. A table of values of $W_{k}$ in the basis case $(k=4)$, using a graph $B_{k}$ having root $v$ and the edges $v u$ and $v w$. The number 61 is always replaced with $a_{k-1}=a_{3}$ so that the resulting function value / lower bound of $W_{k}$ appears in the same form as it does in Table $3.4 \ldots \ldots \ldots 77$

TABLE 3.6. Evidence for Table 3.4 when $k>4$ is even, using a graph $B_{k}$ having root vertex $v$ and the edges $v u$ and $v w$. The relationship $a_{k-1}=2 a_{k-2}+7$ allows us to express the sum in terms of $a_{k-1} \ldots \ldots \ldots \ldots$

TABLE 3.7. Evidence for Table 3.4 when $k>4$ is odd, using a graph $B_{k}$ having root vertex $v$ and the edges $v u$ and $v w$. The relationship $a_{k-1}=2 a_{k-2}+9$ allows us to express the sum in terms of $a_{n-1} \ldots \ldots \ldots . \ldots 80$ 


\section{INTRODUCTION}

\subsection{Traditional Notation and Definitions}

A graph is a set containing a set of vertices $V(G)$ and a set of edges $E(G)$, where each edge represents a connection, or adjacency, between a pair of vertices. Vertices $u$ and $v$ are adjacent if and only if $u v$ is in $E(G)$. The neighbors of $v$ are all the vertices adjacent to $v$. The endvertices of an edge $u v$ are $u$ and $v$. Two edges are incident if they share an endvertex. An edge is incident to a vertex if that vertex is an endvertex of the edge.

All graphs considered in this thesis are finite and simple, meaning the number of vertices is finite, no edge connects a vertex to itself, and there is at most one edge connecting every pair of vertices. The degree of a vertex $v \in V(G)$ is the number of edges in $E(G)$ incident to $v$. This is denoted by $\operatorname{deg}_{G}(v)$ or simply $\operatorname{deg}(v)$ if it is obvious what graph is being examined. The maximum degree $\Delta(G)$ is the highest degree among all vertices in the graph, and the minimum degree $\operatorname{deg}(G)$ is the lowest. A leaf is a vertex of degree 1, and a pendant edge is an edge having one leaf endvertex.

A walk is a sequence of vertices in $V(G)$ such that each vertex is adjacent to the next one in the sequence. A path is a walk in which vertices are never repeated. A graph will be called $P_{n}$ if it is nothing more than a path with $n$ vertices and $n-1$ edges. A cycle is a walk beginning and ending at the same vertex, in which no other vertices are repeated. A graph will be called $C_{n}$, or the $n$-cycle, if it is 
nothing more than a cycle with $n$ vertices and $n$ edges. The length of a walk is one less than the length of its vertex sequence.

The distance $\operatorname{dist}(u, v)$ between two vertices $u$ and $v$ in $V(G)$ is the length of the shortest path in $G$ that includes both $u$ and $v$. If no such path exists, then $\operatorname{dist}(u, v)=\infty$. The graph $G$ is connected if $\operatorname{dist}(u, v)$ is finite for every pair of vertices in $V(G)$. Every graph considered in this thesis is a connected graph.

For every graph $G$, we say that a graph $H$ is a subgraph of $G$ if and only if $V(H) \subseteq V(G)$ and $E(H) \subseteq E(G)$. A tree is a connected graph containing no cycle as a subgraph. The star on $n$ vertices, denoted by $S_{n}$, is a tree that includes a vertex $v$, with the property that every other vertex in $V\left(S_{n}\right)$ is adjacent to $v$. A binary tree is a tree with a special vertex $r$, called the root, and the property that (1) every vertex except $r$ is adjacent to one vertex called its "parent" and (2) every vertex is the parent of zero, one, or two "child" vertices. The full binary tree of height $n$, denoted by $B_{n}$, is a binary tree in which every vertex $v$ has two children if $\operatorname{dist}(v, r)<n$ and no children if $\operatorname{dist}(v, r)=n$.

A subset of vertices $A \subseteq V(G)$ is an independent set of $G$ if no two vertices in $A$ are adjacent. A subset of edges $B \subseteq E(G)$ is a matching in $G$ if no two edges in $B$ are incident. A subset of elements $T \subseteq V(G) \cup E(G)$ is a total independent set if no two vertices in $T$ are adjacent, no two edges in $T$ are incident, and no edge in $T$ has an endvertex in $T$.

The complete graph on $n$ vertices, denoted by $K_{n}$, is a graph with $n$ vertices in which every vertex is a neighbor of every other vertex. The complete bipartite graph $K_{m, n}$ is a graph whose vertices can be partitioned into two independent sets $M$ and $N$, where every vertex in $M$ is adjacent to every vertex in $N$.

The hypercube of dimension $n \geq 0$, also called the $n$-cube and denoted by $Q_{n}$, is a graph in which there are $2^{n}$ vertices, each corresponding to a distinct $n$-tuple in $\mathbb{Z}_{2}^{n}$, and in which two vertices are adjacent if and only if their corresponding 
$n$-tuples differ in exactly one position. The hypercube $Q_{n}$ has $n 2^{n-1}$ edges.

A total coloring $\phi$ of $G$ is a mapping $\phi: V(G) \cup E(G) \rightarrow X$ from the vertices and edges to some set of colors $X$. A total coloring is proper if (1) adjacent vertices have different colors, (2) incident edges have different colors, and (3) every edge has a color that is different from each of its endvertices. Every total coloring in this thesis is assumed to be proper.

For each coloring $\phi$ of $G, \sigma(\phi)$ denotes the total number of different colors that are assigned to elements by $\phi$. A total- $k$-coloring $\phi$ of $G$ is a proper total coloring such that $\sigma(\phi)=k$ (the coloring only uses $k$ colors). The total chromatic number $\chi^{T}(G)$ of a graph $G$ is the smallest number $k$ for which $G$ has a total- $k$ coloring.

A color class is a set of all elements in a graph that are assigned the same color. For each coloring $\phi$ of a graph $G$ and for each color $c$, the notation $\phi_{c}$ will denote the number of elements in $G$ that $\phi$ assigns the color $c$. For example, $\phi_{\text {blue }}$ is equal to the number of elements in the blue color class, and $\phi_{1}$ is the number of elements with "color 1", when colors are enumerated. If colors are enumerated, $\phi_{\sigma}$ will denote the number of elements that are assigned the color $\sigma(\phi)$.

\subsection{New Notation and Definitions}

The set of all total colorings of $G$ is denoted by $\Phi(G)$. For each total coloring $\phi \in \Phi(G)$ and each element $x \in V(G) \cup E(G)$, the color of $x$ in $\phi$ is denoted by $\phi(x)$. For each total coloring $\phi \in \Phi(G)$ and each vertex $v \in V(G)$, the function $\phi^{1}(v)$ denotes the set of colors that $\phi$ assigns to $v$ and its incident edges, and the function $\phi^{2}(v)$ denotes the set of colors that $\phi$ assigns to $v$, its incident edges, and its neighbors. A partial total coloring $\phi$ is one whose domain is a proper subset of $V(G) \cup E(G)$. Very frequently in Chapter 1, we will work on a partial total 
coloring, gradually assigning, erasing, and reassigning colors until each element is assigned a color.

Two elements in $V(G) \cup E(G)$ are called dependent if they are either adjacent or incident. Therefore, a dependent pair of elements cannot be contained in the same total independent set. A subset of elements $A \subseteq V(G) \cup E(G)$ is called dependent if its elements are pairwise dependent.

\subsection{Application Areas of Total Coloring}

Match Scheduling. Suppose we are planning a martial arts tournament, but want to control the way players spend their time when they are not involved in a match. Knowing that the players are prone to aggression, we want to avoid letting two matched players leave the arena at the same time, to avoid an incident. We can't afford to hire a security guard to watch every player, but they are safe as long as they stay in the arena; so we want to keep the players in the arena even when they are not involved in a match. However, regulations insist that the players be allowed some time to leave the arena to have a meal, visit the souvenir shop, get some fresh air, et cetera. In light of this, the planners must give each player a rest period, one particular hour in which they are allowed to leave the arena. As in a traditional scheduling situation, we can represent players with vertices and represent matches with edges, and we can color the edges to represent the time period in which each match occurs. Additionally, we can color each vertex to represent that player's rest period. This amounts to a total coloring of the tournament graph. We must be sure that (1) no pair of incident edges receive the same color, because no player can be in two matches at once, (2) no vertex can be incident to an edge with the same color as the vertex, because no player should have a match during his rest period, and (3) no two adjacent vertices should have 
the same color, because no two matched players should be allowed to wander off at the same time. Thus, the total coloring must be proper. Then, if we want to know the minimum duration of the tournament, we need only find the total chromatic number of this graph.

Network Task Efficiency. Suppose we have a network of computers, servers, facilities, or any group of stations, which can be called nodes, and these nodes are connected to other nodes in the network via physical conductors such as wires or pipelines. A time comes when every facility and every connection must undergo a certain task, such as an update, maintenance, or test to measure its quality or security. Whenever this task is performed on a particular node or connection, that object must be shut down and remain offline until the task is finished. Each task takes the same amount of time, regardless of the node or connection. The administration wishes to get these tasks completed as soon as possible, while adhering to the following rules: (1) No two adjacent nodes can be offline at the same time. (2) When a node is operational, at most one of its connections can be offline at any given time. (3) When a node is offline, all of its connections are required to remain operational to support the network as a whole. The problem is to schedule the tasks for all these nodes and connections so that the process takes the least amount of time. The solution is to represent nodes with vertices and connections with edges, and properly total-color the graph using the fewest colors, where each color represents the interval of time in which an element will be offline as it undergoes the required task.

Art. Of course, one might be trying to color a graph in order to actually make something colorful. A sculptor, painter, or lightshow artist who is trying to produce something with colored dots could decide it is aesthetically pleasing or interesting to keep same-colors apart in a way that corresponds to a proper coloring. In the realm of vertex coloring, many ideas have been introduced for 
additional rules governing color class size or adjacent color pairs, some of which have visual appeal. For example, the harmonious coloring, in which every pair of colors is used on at most one pair of adjacent vertices [10]; or the complete coloring, in which every pair of colors is used on at least one pair of adjacent vertices [9]; or the equitable coloring, in which color class sizes cannot differ by more than one [17]. If the artist wishes to color the lines connecting the dots as well, so that no part of their structure is left uncolored, then a total coloring is necessary. More involved total colorings that have a harmonious, complete, or equitable nature could be adapted for the realm of total coloring, but some basic questions remain open, such as how many colors are required, and how many elements of the structure can be painted a certain color. In any case, an artist interested in having these questions answered for their purposes could learn about what is and is not possible by studying the total coloring of graphs. 


\section{CHAPTER 1 \\ PLANAR GRAPHS AND THE TOTAL COLORING CONJECTURE}

\subsection{History of the Total Chromatic Number}

One easy lower bound for the total chromatic number of a graph $G$ is $\Delta(G)+1$, because a vertex with degree $\Delta(G)$ needs a color and a unique color for every one of its incident edges. Graphs with the property $\chi^{T}=\Delta+1$ are called Class 1. Some graphs, such as cycles of length $n \neq 0(\bmod 3)$ and complete bipartite graphs of the form $K_{n, n}$, require $\Delta+2$ colors [29]. However, no graph has yet been found with total chromatic number more than $\Delta+2$. This observation lead Vizing [26, 27], and independently, Mehdi Behzad [3], to posit the Total Coloring Conjecture:

$$
\chi^{T}(G) \leq \Delta(G)+2
$$

for any graph $G$, where $\Delta(G)$ is the maximum degree of $G$.

This Conjecture has been confirmed for many families of graphs. Different graphs require different proofs depending on their maximum degree. For graphs

with $\Delta \leq 3$, the conjecture was proven by Rosenfeld [22], and for graphs with $\Delta=4$ and 5, it was proved by Kostochka [14, 13]. Borodin [4] proved it for planar graphs with $\Delta \geq 9$, and later for planar graphs with $\Delta=8$, as explained in [11]. Sanders and Zhao [23] proved it for planar graphs with $\Delta=7$.

But when graph theorists look at planar graphs with $\Delta=6$, they seem to hit a brick wall. The procedure used for the $\Delta=4$ and 5 cases were increasingly 
complex, and the discharging technique used for the $\Delta=7$ case does not seem powerful enough to extend to all planar $\Delta=6$ graphs.

As Sanders and Zhao put it in the paper [23] in which they first prove the $\Delta=7$ case: "[It] will only increase in difficulty, as [the $\Delta=6$ ] class of graphs includes large graphs almost every vertex of which has degree equal to the maximum degree of the graph". To demonstrate the truth of this, let $I_{0}$ be the graph of the icosahedron, and then, for every $k \geq 0$, define $I_{k+1}$ as a copy of $I_{k}$, with a new vertex inserted in the middle of every edge, plus an edge connecting every new vertex to every other new vertex with which it shares a face. (See Figure 1.1 for an illustration of $I_{1}$ ). Every graph in the family $\left\{I_{k} \mid k \in \mathbb{N}\right\}$ is planar, has maximum degree 6 , and has minimum degree 5 . As $k \rightarrow \infty$, the number of vertices in $I_{k}$ increases without bound, while vertices of degree 5 remain at twelve in number, and grow arbitrarily distant from one another.

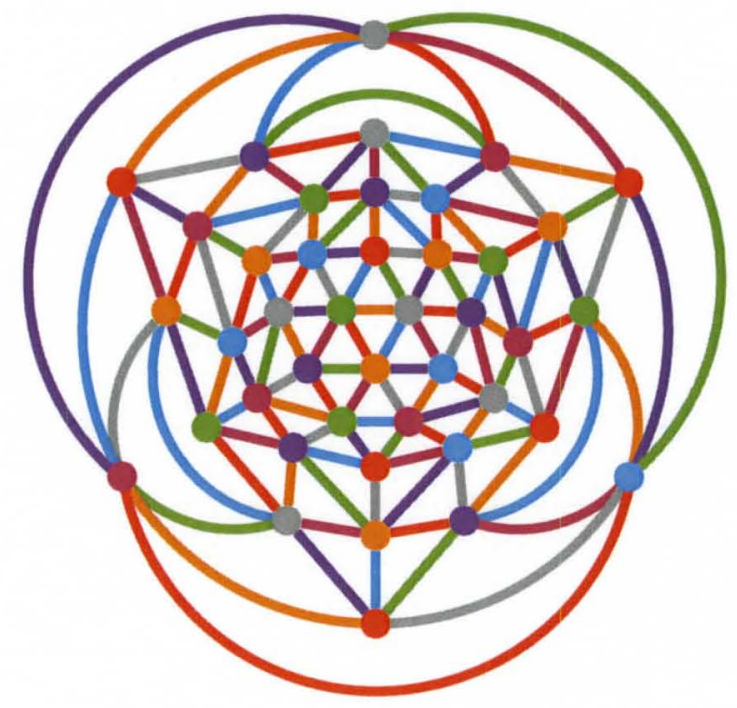

FIGURE 1.1: A total-7-coloring of the graph $I_{1}$. 
Those who attempt the $\Delta=6$ problem inevitably narrow their quarry to an even more restrictive set of graphs. For example, it was proved in [25] that planar, $\Delta=6$ graphs in which no two triangles share an edge satisfy $\chi^{T} \leq 8$, and in [24] it was proved that planar, $\Delta=6$ graphs containing no 4-cycles satisfy $\chi^{T}=7$. These authors used the discharging technique, which was used to solve the $\Delta=7$ case and was also used in [2] to solve the Four-color Theorem. This technique was first officially used in 1904 by Wernicke to prove that every planar graph with minimum degree 5 must have an edge with one endvertex of degree 5 and the other with degree 5 or 6 [21]. It can be very effective in proving the existence of graphical configurations which seem impossible to locate in arbitrary graphs, but must logically exist somewhere.

We will now use this technique to prove the Total Coloring Conjecture for another family of planar, $\Delta=6$ graphs, as it is done in [16]. First, we will choose an arbitrarily counterexample $K$ of smallest size. Then, in the lemmas to follow, it will be shown that some graphical configurations cannot exist in $K$. Finally, a discharging argument will show that one of those configurations must exist in $K$, or else $K$ cannot exist.

\subsection{A Larger Family of $\Delta=6$ Graphs that Satisfy the Conjecture}

For a planar embedding of a graph $G$, let $F(G)$ be the set of all faces. For any face $f \in F(G)$, define its degree $\operatorname{deg}(f)$ to be the length of a walk around its boundary.

For each natural number $n$, define an $n$-vertex to be a vertex of degree $n$. Analogous notation will be used for faces and the term triangle may be used for a 3 -face. An edge connecting an $n_{1}$-vertex to an $n_{2}$-vertex will be called an $\left(n_{1}, n_{2}\right)$-edge, and a face with degree $n$ will be called a $\left(a_{1}, a_{2}, \ldots, a_{n}\right)$-face if a walk 
around its boundary elicits an $a_{1}$-vertex, an $a_{2}$-vertex, and so on, in that order. If a number is raised to a plus or minus sign, it weakens the constraints of the element in question. An $n^{+}$-vertex can refer to any vertex of degree $n$ or more, a $\left(n_{1}, n_{2}^{-}\right)$-edge can refer to any edge that connects an $n_{1}$-vertex to a vertex of degree $n_{2}$ or less, and an $\left(a_{1}, a_{2}^{+}, a_{3}\right)$-triangle refers to a 3 -face surrounded by a vertex of degree $a_{1}$, a vertex of degree $a_{2}$ or more, and a vertex of degree $a_{3}$.

For each pair of natural numbers $n$ and $k$, let $v_{k}^{n}$ be the number of $n$-vertices in $G$ that lie on $k$ distinct 3-cycles in $G$, and let $v_{n}^{k^{+}}$be the number of $n$-vertices that lie on $k$ or more distinct 3-cycles.

Theorem 1. A planar graph $G$ satisfies $\chi^{T}(G) \leq \Delta(G)+2$ as long as

$$
v_{5}^{4}+2\left(v_{5}^{5^{+}}+v_{6}^{4}\right)+3 v_{6}^{5}+4 v_{6}^{6^{+}}<24 .
$$

\subsection{Supporting Lemmas for Theorem 1}

Define $\Gamma$ to be the set of all possible counterexamples to this theorem. In other words, $\Gamma$ is the family of all planar graphs that satisfy (1.2) but not (1.1). Since it is known that planar graphs with $\Delta \geq 7$ satisfy (1.1), as do all graphs with $\Delta \leq 5$, it must be that every graph in $\Gamma$ has $\Delta=6$. Let $K$ be a graph in $\Gamma$ with a minimum number of edges. Choose a particular planar embedding for $K$ so that $F(K)$ is defined.

Since $K$ does not satisfy (1.1), we know $\chi^{T}(K) \geq 9$. Note, however, that every proper subgraph $H$ of $K$ will have the property $\chi^{T}(H) \leq 8$. A proper subgraph is the result of deleting elements, so $H$ must have fewer edges than $K$, and this implies it cannot be in $\Gamma$. But a subgraph of a planar graph must be planar, and will still satisfy (1.2) because the removal of edges cannot increase the left side of that inequality. Therefore, the exclusion of $H$ from $\Gamma$ must imply that $H$ satisfies (1.1). Therefore, $\chi^{T}(H) \leq \Delta(H)+2 \leq \Delta(K)+2=8$. 
There are some configurations that $K$ cannot have in its structure. For example, a leaf $v$ cannot exist in $K$. If it did, then we could total-8-color the subgraph $K-v$, apply those colors to their corresponding elements of $K$, give the edge to $v$ a color from $N_{8}$ (the colors 1 through 8 ) that doesn't conflict with nearby colors, and then give $v$ a color that doesn't conlict with either its neighbor or their shared edge. This produces a total-8-coloring of $K$, which is impossible.

Graph theorists call a configuration "reducible" if its existence in a minimum counterexample would lead to a reduction of its chromatic number, thereby showing that the graph is not really a counterexample. The following set of lemmas will address some other reducible configurations.

Lemma 1. $K$ does not contain a vertex of degree 2 or less.

Proof. Suppose it does. We have seen that a 1 -vertex cannot be in $K$, so assume instead that some 2-vertex $v$ exists, with neighbors labeled $u$ and $w$. Total-8-color $K-v u$, and create a partial total coloring $\phi$ that assigns those colors to that subgraph in $K$, leaving vertex $v$ and both of its incident edge(s) uncolored. Let $\phi$ assign $u v$ a color from $N_{8} \backslash \phi^{1}(u)$. Then assign $u w$ a color from $N_{8} \backslash \phi^{1}(w) \backslash \phi(u v)$ and assign $v$ a color from $N_{8} \backslash \phi^{2}(v)$ to complete the coloring. Now $K$ is total-8colored, which is impossible.

Lemma 2. $K$ does not contain a $\left(3,5^{-}\right)$-edge.

Proof. Suppose it does. Let $y$ be the 3-vertex and let $z$ be its neighbor of degree 5 or less. Total-8-color the subgraph $K-y z$ and create a partial total coloring $\phi$ that assigns those colors to that subgraph in $K$, leaving $y$ and $y z$ uncolored. Let $\phi$ assign $y z$ a color from $N_{8} \backslash \phi^{1}(y) \backslash \phi^{1}(z)$, then give $y$ a color from $N_{8} \backslash \phi^{2}(y)$ to complete the coloring. Now $K$ is total-8-colored, which is impossible. 

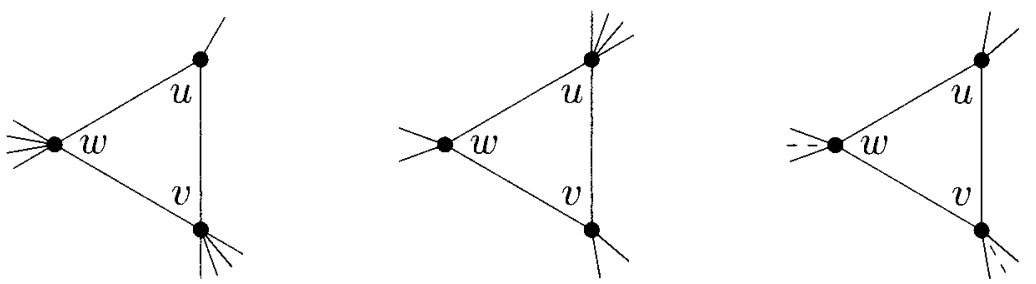

FIGURE 1.2: A $(3,6,6)$-triangle, a $(4,4,6)$-triangle, and a $\left(4,5^{-}, 5^{-}\right)$-triangle.

Lemma 3. $K$ does not contain a $(3,6,6)$-triangle.

Proof. Suppose it does. Let $u$ be the 3-vertex, let $v$ and $w$ be the two 6 -vertices, and let $u^{\prime}$ be the third neighbor of $u$. Total-8-color the subgraph $K-u$ and create a partial total coloring $\phi$ that assigns those colors to that subgraph in $K$, leaving $u$ and its incident edges uncolored. If there is a color from $N_{8}$ that is in neither $\phi^{1}(v), \phi^{1}(w)$, nor $\phi^{1}\left(u^{\prime}\right)$, reassign $v w$ that color in $\phi$. In every case, $\left(\phi^{1}(v) \cup \phi^{1}(w)\right) \backslash \phi^{1}\left(u^{\prime}\right)$ results in a non-empty set. Define 1 to be a color from that set, and without loss of generality, $1 \in \phi^{1}(v)$. Give $u u^{\prime}$ color 1 , then give $u w$ a color from $N_{8} \backslash\{1\} \backslash \phi^{1}(w)$, give $u v$ a color from $N_{8} \backslash \phi(u w) \backslash \phi^{1}(v)$, and finally give vertex $u$ a color from $N_{8} \backslash \phi^{2}(u)$ to complete the coloring. Now $K$ is total-8-colored, which is impossible.

Lemma 4. $K$ does not contain a $(4,4,6)$-triangle.

Proof. Suppose it does. Let $u$ be the 6 -vertex and let $v$ and $w$ be the two 4 -vertices, as shown in the middle of Figure 1.2. Total-8-color the subgraph $K-w v$ and create a partial total coloring $\phi$ that assigns those colors to that subgraph in $K$, leaving $v$ and $v w$ uncolored. Let $\phi$ assign $v$ a color from $N_{8} \backslash \phi^{2}(v)$. If $\phi^{1}(w) \cup \phi^{1}(v) \neq N_{8}$, we could assign $v w$ a color that's not in the union to complete the total-8-coloring. Assume, instead, that $\phi^{1}(w) \cup \phi^{1}(v)=N_{8}$. Each set in this union has four colors, so none can be shared. Let $n$ be a color in the set $N_{8} \backslash \phi^{1}(u)$. Color $n$ is in either $\phi^{1}(w)$ or $\phi^{1}(v)$, but not both, so without loss of generality, assume 
$n \in \phi^{1}(w)$. Assign $v w$ the same color as $u v$, then reassign $u v$ color $n$. Now $K$ is total-8-colored, which is impossible.

Lemma 5. $K$ does not contain a $\left(4,5^{-}, 5^{-}\right)$-triangle.

Proof. Suppose it does. Let $u$ be the 4-vertex, and let $v$ and $w$ be its neighbors on the triangle, as shown on the right in Figure 1.2. Total-8-color the subgraph $K-u v$ and create a partial total coloring $\phi$ that assigns those colors to that subgraph in $K$, leaving $u$ and $u v$ uncolored. Let $\phi$ assign $u$ a color from $N_{8} \backslash \phi^{2}(u)$, then define colors $\phi(v w)=1$ and $\phi(u w)=2$. If some color in $N_{8}$ is absent from $\phi^{1}(u) \cup \phi^{1}(v)$, the total-8-coloring can be finished by assigning $u v$ that color.

Assume $\phi^{1}(u) \cup \phi^{1}(v)=N_{8}$. This implies either $1 \notin \phi^{1}(u)$ or $2 \notin \phi^{1}(v)$. Suppose both are true: Define $\phi^{1}(u)=\{2,3,4,5\}$; then $\{6,7,8\} \subseteq \phi^{1}(v)$. If $\phi^{1}(w)$ lacks a color from $\{6,7,8\}$, we could reassign $u w$ that color and give $u v$ color 2 to finish the coloring. Otherwise, reassign $v w$ a color from $\{3,4,5\} \backslash \phi^{1}(v) \backslash \phi^{1}(w)$, then assign $u v$ color 1 to finish the coloring.

Assume that either $1 \in \phi^{1}(u)$ or $2 \in \phi^{1}(v)$ (but not both). Suppose $1 \in \phi^{1}(u)$ but $2 \notin \phi^{1}(v)$ : Define $\phi^{1}(u)=\{1,2,3,4\}$; then $\{5,6,7,8\} \subseteq \phi^{1}(v)$. If $\phi^{1}(w)$ lacks a color from $\{5,6,7,8\}$, we could reassign $u w$ that color and give $u v$ color 2 to finish the coloring. Otherwise, erase the colors of $v w, u w$, and $u$, give $u v$ the color of $v$, reassign $v$ a color from $N_{8} \backslash \phi^{2}(v)$, and give $u$ a color from $N_{8} \backslash \phi^{2}(u)$. Since $\phi(u v) \in \phi^{1}(w)$, we can give $u w$ a color from $N_{8} \backslash \phi^{1}(u) \backslash \phi^{1}(w)$ and then give $v w$ a color from $N_{8} \backslash \phi^{1}(v) \backslash \phi^{1}(w)$ to finish the coloring.

Assume, finally, that $1 \notin \phi^{1}(u)$ and $2 \in \phi^{1}(v)$. Define $\phi^{1}(u)=\{2,6,7,8\}$; then $\{3,4,5\} \subseteq \phi^{1}(v)$. If $\phi^{1}(w)$ lacks a color from $\{6,7,8\}$, we could reassign $v w$ that color and give $u v$ color 1 to finish the coloring. Otherwise, give $u v$ the color of $u$, erase the color from $u w$, reassign $u$ a color from $N_{8} \backslash \phi^{2}(u)$, and give $u w$ a color from $\{3,4,5\} \backslash \phi(u) \backslash \phi^{1}(w)$ to finish the coloring. Now $K$ is total-8-colored, which is impossible. 

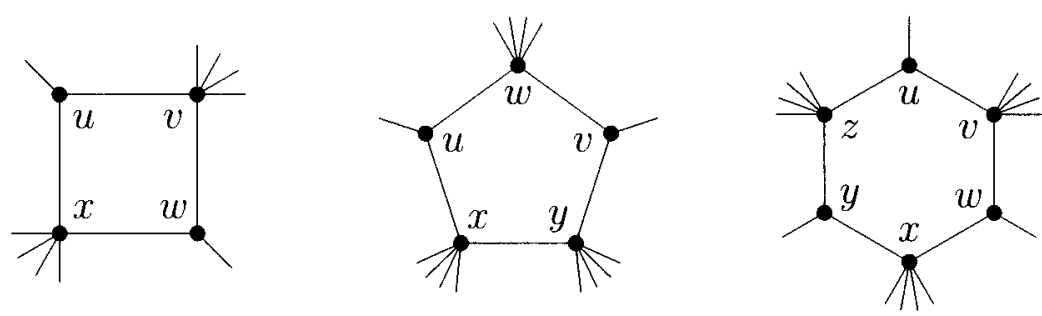

FIGURE 1.3: A 4-face with two 3-vertices, a 5-face with two 3-vertices, and a 6-face with three 3 -vertices.

Lemma 6. $K$ does not contain a 4-face that has two or more 3-vertices on its boundary.

Proof. Suppose it does. Let $u$ and $w$ be the two 3-vertices on the boundary of the 4-face. By Lemma 2, $u$ and $w$ cannot be adjacent, and every other vertex on the boundary must be a 6 -vertex. Label the vertices on the boundary of that $(3,6,3,6)$ face as shown on the left in Figure 1.3. Let $u^{\prime}$ and $w^{\prime}$ denote the respective neighbors of $u$ and $w$ that are not on the boundary of the 4-face. Total-8-color the subgraph $K-v w$ and create a partial total coloring $\phi$ that assigns those colors to that subgraph in $K$, leaving $w, x w$, and $v w$ uncolored.

Suppose $\phi^{1}(x) \neq \phi^{1}(v)$. If $\phi\left(w w^{\prime}\right) \in \phi^{1}(x)$, give $v w$ a color from $N_{8} \backslash \phi^{1}(v) \backslash$ $\phi\left(w w^{\prime}\right)$, then give $w x$ a color from $N_{8} \backslash \phi^{1}(x) \backslash \phi(v w)$. If $\phi\left(w w^{\prime}\right) \in \phi^{1}(v) \backslash \phi^{1}(x)$, give $x w$ a color from $N_{8} \backslash \phi^{1}(x) \backslash \phi\left(w w^{\prime}\right)$, then give $v w$ a color from $N_{8} \backslash \phi^{1}(v) \backslash$ $\phi(v w)$. If $\phi\left(w w^{\prime}\right)$ is in neither $\phi^{1}(x)$ nor $\phi^{1}(y)$, give $x w$ a color from $\phi^{1}(v) \backslash \phi^{1}(x)$, and give $v w$ a color from $\phi^{1}(x) \backslash \phi^{1}(v)$. After every case, we can give $w$ a color from $N_{8} \backslash \phi^{2}(w)$ to finish the coloring.

Assume $\phi^{1}(x)=\phi^{1}(v)$. Remove the color of $u$. Reassign $w x$ color $\phi(u x)$, reassign $u x$ a color from $N_{8} \backslash \phi^{1}(x) \backslash \phi^{1}\left(u u^{\prime}\right)$, give $v w$ a color from $N_{8} \backslash \phi^{1}(v) \backslash$ $\phi^{1}\left(w w^{\prime}\right)$, give $w$ a color from $N_{8} \backslash \phi^{2}(w)$, and then give $u$ a color from $N_{8} \backslash \phi^{2}(u)$ to finish the coloring. Now $K$ is total-8-colored, which is impossible. 
Lemma 7. $K$ does not contain a 5-face that has two or more 3-vertices on its boundary.

Proof. Suppose it does. Let $u$ and $v$ be two 3 -vertices on the boundary of the 5 -face. By Lemma 2, $u$ and $v$ cannot be adjacent, and every other vertex on the boundary must be a 6 -vertex. Label the vertices on the boundary of that $(3,6,3,6,6)$-face as shown in the middle of Figure 1.3. Let $u^{\prime}$ and $v^{\prime}$ denote the respective neighbors of $u$ and $v$ that are not on the boundary of the 5-face. Total-8-color the subgraph $K-u-v$ and create a partial total coloring $\phi$ that assigns those colors to that subgraph in $K$, leaving $u, v$, and their incident edges uncolored.

Suppose either $\phi^{1}(y) \neq \phi^{1}\left(v^{\prime}\right)$ or $\phi^{1}(x) \neq \phi^{1}\left(u^{\prime}\right)$; without loss of generality, assume the first statement is true. Give $u x$ a color from $N_{8} \backslash \phi^{1}(x)$, then give $u u^{\prime}$ a color from $N_{8} \backslash \phi^{1}(x) \backslash \phi(u x)$, give $u w$ a color from $N_{8} \backslash \phi^{1}(u) \backslash \phi^{1}(w)$, and give $u$ a color from $N_{8} \backslash \phi^{2}(u)$. Since $\left|\phi^{1}(y) \cup \phi^{1}\left(v^{\prime}\right)\right| \geq 7$, we can then give $v w$ a color from $\left(\phi^{1}(y) \cup \phi^{1}\left(v^{\prime}\right)\right) \backslash \phi^{1}(w)$. Now either $\phi^{1}\left(v^{\prime}\right)$ or $\phi^{1}(y)$ includes $\phi(v w)$. If $\phi(v w) \notin \phi^{1}(y)$, give $v y$ a color from $N_{8} \backslash \phi^{1}(y) \backslash \phi(v w)$, then give $v v^{\prime}$ a color from $N_{8} \backslash \phi^{1}\left(v^{\prime}\right) \backslash \phi^{1}(v)$; otherwise, give $v v^{\prime}$ a color from $N_{8} \backslash \phi^{1}\left(v^{\prime}\right) \backslash \phi(v w)$, then give $v y$ a color from $N_{8} \backslash \phi^{1}(y) \backslash \phi^{1}(v)$. Give $v$ a color from $N_{8} \backslash \phi^{2}(v)$ to finish the total-8-coloring.

Assume, instead, that $\phi^{1}(y)=\phi^{1}\left(v^{\prime}\right)$ and $\phi^{1}(x)=\phi^{1}\left(u^{\prime}\right)$. Then suppose either $\phi^{1}(w) \nsubseteq \phi^{1}(x)$ or $\phi^{1}(w) \nsubseteq \phi^{1}(y)$; without loss of generality, assume the first statement is true. Define 1 to be a color from $\phi^{1}(w) \backslash \phi^{1}(x)$. Give $u x$ color 1 and give $u u^{\prime}$ a color from $N_{8} \backslash \phi^{1}\left(u^{\prime}\right) \backslash\{1\}$. Give $v y$ a color from $N_{8} \backslash \phi^{1}(y)$, give $v v^{\prime}$ a color from $N_{8} \backslash \phi^{1}\left(v^{\prime}\right) \backslash \phi(v y)$, give $v w$ a color from $N_{8} \backslash \phi^{1}(w) \backslash \phi^{1}(v)$, and give $v$ a color from $N_{8} \backslash \phi^{2}(v)$. Now $\left|\phi^{1}(w) \cup \phi^{1}(u)\right| \leq 7$, so give $u w$ a color from $N_{8} \backslash \phi^{1}(u) \backslash \phi^{1}(w)$, and give $u$ a color from $N_{8} \backslash \phi^{2}(u)$ to finish the total-8-coloring.

Now assume all five colors in $\phi^{1}(w)$ exist in both $\phi^{1}(x)$ and $\phi^{1}(y)$. Each of those sets can have only one color not in $\phi^{1}(w)$, so their union $A=\phi^{1}(x) \cup \phi^{1}(y)$ 
has at most seven colors. Define 1 to be a color from $N_{8} \backslash A$. Give $u u^{\prime}$ and $v v^{\prime}$ color 1 , and reassign $x y$ color 1 . Give $u w$ a color from $N_{8} \backslash \phi^{1}(w) \backslash\{1\}$, and give $v w$ a color from $N_{8} \backslash \phi^{1}(w) \backslash\{1\} \backslash \phi(u w)$. Give $u x$ a color from $N_{8} \backslash \phi^{1}(u) \backslash \phi^{1}(x)$, give $v y$ a color from $N_{8} \backslash \phi^{1}(v) \backslash \phi^{1}(y)$, give $u$ a color from $N_{8} \backslash \phi^{2}(u)$, and give $v$ a color from $N_{8} \backslash \phi^{2}(v)$ to finish the coloring. Now $K$ is total-8-colored, which is impossible.

Lemma 8. $K$ does not contain a 6-face that has three or more 3-vertices on its boundary.

Proof. Suppose it does. Let $u, w$, and $y$ be three 3-vertices on the boundary of the 6-face. By Lemma 2, no pair of those vertices can be adjacent, and every other vertex on the boundary must be a 6 -vertex. Label the vertices on the boundary of that $(3,6,3,6,3,6)$-face as shown on the right in Figure 1.3. Let $u^{\prime}, w^{\prime}$, and $y^{\prime}$ denote the respective neighbors of $u, w$, and $y$ that are not on the boundary of the 6-face. Total-8-color the subgraph $K-u-w-y$ and create a partial total coloring $\phi$ that assigns those colors to that subgraph in $K$, leaving $u, w, y$, and their incident edges uncolored. Give $u z$ a color from $\phi^{1}\left(u^{\prime}\right) \backslash \phi^{1}(z)$, give $v w$ a color from $\phi^{1}\left(w^{\prime}\right) \backslash \phi^{1}(v)$, and give $x y$ a color from $\phi^{1}\left(y^{\prime}\right) \backslash \phi^{1}(x)$. For each edge $a b \in\{u v, w x, y z\}$, give $a b$ a color from $N_{8} \backslash \phi^{1}(a) \backslash \phi^{1}(b)$. For each edge $a b \in\left\{u u^{\prime}, w w^{\prime}, y y^{\prime}\right\}$, give $a b$ a color from $N_{8} \backslash \phi^{1}(a) \backslash \phi^{1}(b)$. For each vertex $b \in\{u, w, y\}$, give $b$ a color from $N_{8} \backslash \phi^{2}(b)$. This completes the total-8-coloring of $K$, which is impossible.

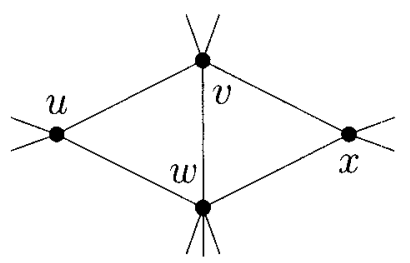

FIGURE 1.4: A pair of $(4,5,6)$-triangles that share a common $(5,6)$-edge. 
Lemma 9. $K$ does not contain a pair of $(4,5,6)$-triangles that share a common $(5,6)$-edge.

Proof. Suppose it does. Label the 5-vertex $v$, the 6-vertex $w$, and the two 4 -vertices $u$ and $x$, as shown on the left in Figure 1.4. Let $u^{\prime}$ and $u^{\prime \prime}$ be the other neighbors of $u$, and let $v^{\prime}$ and $v^{\prime \prime}$ be the other neighbors of $v$. Total-8-color the subgraph $K-u v$ and create a partial total coloring $\phi$ that assigns those colors to that subgraph in $K$, leaving $u$ and $u v$ uncolored. Then give $u$ a color from $N_{8} \backslash \phi^{2}(u)$. Define $\phi(v w)=1$ and $\phi(u w)=2$. If $\phi^{1}(u) \cup \phi^{1}(v)$ is missing a color from $N_{8}$, we could give that color to $u v$ to finish the total-8-coloring.

Assume $\phi^{1}(u) \cup \phi^{1}(v)=N_{8}$. Given the cardinalities of those sets, there is a unique color $m$ such that $m \in \phi^{1}(u) \cap \phi^{1}(v)$. Consider the possible values of $m$.

Case 1: $m>2$. Define $\phi^{1}(u)=\{2,3,4,5\}$; this implies $\{6,7,8\} \subseteq \phi^{1}(v)$. If $\phi^{1}(w)$ lacks a color from $\{6,7,8\}$, we could reassign $u w$ that color and give $u v$ color 2 to finish the coloring. Or, if $\phi^{1}(w)$ lacks a color from $\{3,4,5\} \backslash\{m\}$, we could reassign $v w$ that color and give $u v$ color 1 to finish the coloring.

Assume otherwise: $\phi^{1}(w)=N_{8} \backslash\{m\}$. If $m=\phi(u)$, reassign $u w$ color $m$. Then, if $u$ has no neighbor of color 1 , reassign $u$ color 1 and give $u v$ color 2; otherwise, reassign $v w$ color 2 , give $u v$ color 1 , and then reassign $u$ a color from $N_{8} \backslash \phi^{2}(u)$. Either way finishes the total-8-coloring.

Assume, instead, that $m \neq \phi(u)$. Give $u v$ color $\phi(u)$. If $u$ has a neighbor of color 2, then it can be reassigned a color from $N_{8} \backslash \phi^{2}(u)$; otherwise, change its color to 2, reassign $u w$ color 1 , and reassign $v w$ color 2 ; this completes the total-8-coloring.

Case 2: $\quad m=2$. Define $\phi^{1}(u)=\{2,3,4,5\}$; this implies $\{6,7,8\} \subseteq \phi^{1}(v)$. If $\phi(v)=2$, we could give $u v$ color $\phi(u)$ and reassign $u$ a color from $N_{8} \backslash \phi^{2}(u)$ to complete the coloring; if $\phi(v) \neq 2$ but a color from $\{3,4,5\}$ isn't in $\phi^{1}(w)$, we could 
reassign $v w$ the missing color, then give $u v$ color 1 to complete the coloring.

Assume neither is true: $\phi(v) \neq$, and $\{3,4,5\} \subseteq \phi^{1}(w)$. Give $u v$ color $\phi(u)$. If $u$ has a neighbor of color 2 , reassign it a color from $N_{8} \backslash \phi^{2}(u)$; otherwise, reassign $u w$ a color from $\{6,7,8\} \backslash \phi^{1}(w)$ and reassign $u$ color 2 ; this completes the total-8-coloring.

Case 3: $m=1$. Define $\phi^{1}(u)=\{1,2,3,4\}$; this implies $\{5,6,7,8\} \subseteq \phi^{1}(v)$. If $\phi^{1}(w)$ lacks a color from $\{5,6,7,8\}$, we could reassign $u w$ that color and give $u v$ color 2 to finish the coloring.

Assume otherwise: $\{5,6,7,8\} \subseteq \phi^{1}(w)$. Now $\phi^{1}(w)$ contains either 3 or 4 , but not both; without loss of generality, let 4 be the one contained: $\phi^{1}(w)=$ $N_{8} \backslash\{3\} .$. The color of $u$ is either 1,3 , or 4 . If $\phi(w)=4$, we could reassign $v w$ color 3 , give $u v$ color 1 , and reassign $u$ a new color to finish the coloring.

Assume that $\phi(w) \neq 4$; without loss of generality we will define $\phi(w)=7$ and $\phi(v)=8$. Now, if color $n \in\{5,6\}$ does not appear on a neighbor of $u$, give $u v$ the color $\phi(u)$, reassign $u$ color $n$, and if $\phi(u v)=1$, reassign $v w$ color 3 ; this would finish the coloring.

Assume that colors 5 and 6 both appear on neighbors of $u$. If $\phi(u)=1$, give $u v$ color 8 , erase the colors on $u$ and $u w$ and reassign $v$ a color from $N_{8} \backslash \phi^{2}(v)$, erase the color of $u w$ and give both $u$ and $v w$ some color from $\{1,2\} \backslash \phi(v)$, and then give $u w$ a color from $\{1,2\} \backslash \phi(u)$. If, instead, $\phi(u)=3$, give $u v$ color 8 , erase the colors on $u$ and $u w$ and reassign $v$ a color from $N_{8} \backslash \phi^{2}(v)$, then give $u$ and $v w$ a color from $\{2,3\} \backslash \phi(v)$, and if that color is 2 , reassign $u w$ color 2 . In both cases, the total-8-coloring is finished.

Assume that $\phi(u)=4$. If a color from $\{1,2,3\}$ does not appear on a neighbor of $v$, we could assign $v$ that color and change $\phi(v w)$ to 3 if necessary, then give $u v$ color 8 to finish the coloring.

Finally, assume that colors 1, 2, and 3 all appear on neighbors of $v$. Remove 
the colors on $u, u v, v, v x$, and $x$. Reassign $v w$ a color from $\{1,3\}$ that is already a color of a neighbor of $v$. Let $j$ be a color from $\{1,2,3\}$ that isn't used on a neighbor of $v$. If $j \in \phi^{1}(x)$, give $v$ color $j$; otherwise, give $v x$ color $j$. Give $x$ a color from $N_{8} \backslash \phi^{2}(x)$. If $\phi(v)=j$, give $v x$ a color from $N_{8} \backslash \phi^{1}(v) \backslash \phi^{1}(x)$; otherwise, give $v$ a color from $N_{8} \backslash \phi^{2}(v)$. Give $u$ a color from $N_{8} \backslash \phi^{2}(u)$, and give $u v$ a color from $N_{8} \backslash \phi^{1}(u) \backslash \phi^{1}(v)$. Now $K$ is total-8-colored, which is impossible.
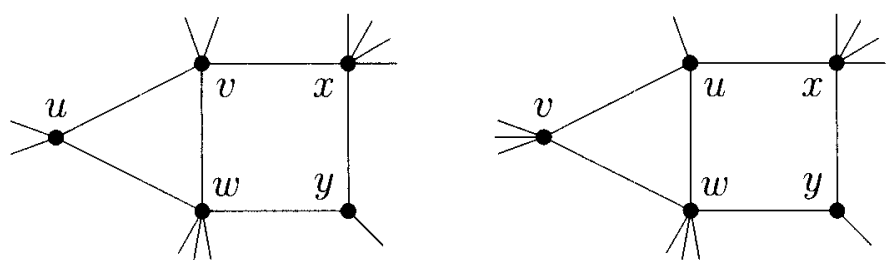

FIGURE 1.5: A $(4,5,6)$-triangle sharing an edge with a $\left(3,6,5^{-}, 6\right)$-face. On the left, the $(5,6)$-edge is shared; on the right, the $(4,6)$-edge is shared.

Lemma 10. $K$ does not contain a $(4,5,6)$-triangle that shares an edge with a $\left(3,6,5^{-}, 6\right)$-face.

Proof. Suppose it does. Label the 4-vertex, 5-vertex, and 6-vertex of the triangle $u, v$, and $w$, respectively; label the 3-vertex $y$, and label the 6-vertex on the 4-face $x$. Total-8-color the subgraph $K-u v$ and create a partial total coloring $\phi$ that assigns those colors to that subgraph in $K$, leaving $u$ and $u v$ uncolored. Then give $u$ a color from $N_{8} \backslash \phi^{2}(u)$. Define $\phi(v w)=1$ and $\phi(u w)=2$. Following the proof of Lemma 9 until the last paragraph begins, we see that $K$ can be total8-colored in every situation except the following: $\phi(v)=8, \phi(w)=7, \phi(u)=4$, $\phi^{1}(v)=\{1,5,6,7,8\}, \phi^{1}(w)=N_{8} \backslash\{3\}, \phi^{1}(u)=\{1,2,3,4\}, v$ has neighbors of color 1,2 , and 3 , and $u$ has neighbors of color 5 and 6 . We will assume all this to be true. As shown in Figure 1.5, either a $(5,6)$-edge or a $(4,6)$-edge is shared by the two faces. 
Case 1: The faces share a $(5,6)$-edge. Remove the colors on $u$ and $y$. If color 1 , 2 , or 3 isn't used on a neighbor of $w$, reassign $w$ that color (and reassign $v w$ color 3 if $\phi(w)=1$, then reassign $u w$ color 7 , reassign $u$ color 2 , give $u v$ color 4 , and give $y$ a color from $N_{8} \backslash \phi^{2}(y)$ to finish the coloring.

Assume the colors 1, 2, and 3 appear on neighbors of $w$. If a color $n \in\{1,3\}$ isn't in $\phi^{1}(y)$, reassign $u w$ color $\phi(w y)$, reassign $w y$ color $n$ (and reassign $v w$ color 3 if $n=1$ ), reassign $u$ color 2 , give $u v$ color 4 (then switch the colors of $u v$ and $v$ if $\phi(u w)=4$ ), and give $y$ a color from $N_{8} \backslash \phi^{2}(y)$ to finish the coloring.

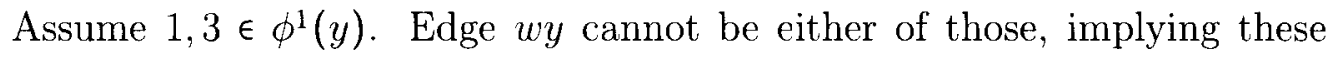
colors are used on the other two edges incident to $y$. Switch the colors on $w$ and $w y$; now $\phi(w y)=7$. Reassign $u$ color 7 , reassign $u v$ color 4 (then switch the colors of $u v$ and $v$ if $\phi(u w)=4$ ), and give $y$ a color from $N_{8} \backslash \phi^{2}(y)$. Now $K$ is total-8-colored, which is impossible.

Case 2: The faces share a (4,6)-edge. Remove the color of $y$. If $\phi(u x) \notin \phi^{1}(y)$, reassign $u w$ color $\phi(w y)$ (then switch the colors of $v$ and $u$ if $\phi(w y)=4$ ), reassign $w y$ color $\phi(u x)$, reassign $v w$ a color from $\{1,3\} \backslash \phi(u x)$, give $u v$ color 2 , and give $y$ a color from $N_{8} \backslash \phi^{2}(y)$ to finish the coloring.

Assume $\phi(u x) \in \phi^{1}(y)$. Make sure $\phi(x y) \neq 2$ by assigning it a color from $N_{8} \backslash \phi^{1}(x) \backslash\{2\}$. Now switch the colors on $u w$ and $w y$, reassign $u$ a color from $\{4,8\} \backslash \phi(u w)$, reassign $v$ a color from $\{4,8\} \backslash \phi(u)$, give $u v$ color 2 , and give $y$ a color from $N_{8} \backslash \phi^{2}(y)$ to finish the coloring. Now $K$ is total-8-colored, which is impossible. 


\subsection{Discharging}

These lemmas give us a lot of information about the structure of $K$, such as what sort of configurations to expect, and this will be used to control the distribution of charges that are placed on the graph and then redistributed. For example, they allow us to make the following important remark:

Two special vertices of $K$ cannot lie on the same face.

The reason for this is easily explained. By definition, a special vertex cannot lie on a $5^{+}$-face, and by Lemma 3 , it cannot lie on a triangle. Therefore, every face incident to a special vertex is a 4 -face. Observing Lemma 6, no 4-face can have more than one special vertex on its boundary. Therefore, no pair of special vertices can lie on the boundary of a single face.

Now we are ready to present a proof of Theorem 1.

Proof. Give each vertex and face in $K$ a charge equal to its degree minus 4 , and add up the total charge:

$$
\begin{gathered}
\sum_{v \in V(G)}(\operatorname{deg}(v)-4)+\sum_{f \in F(K)}(\operatorname{deg}(v)-4) \\
=\sum_{v \in V(K)} \operatorname{deg}(v)+\sum_{f \in F(K)} \operatorname{deg}(f)-4|V(K)|-4|F(K)| .
\end{gathered}
$$

Applying the Handshaking Lemma to the first two terms and Euler's formula to the second two terms, we see that the total charge is equal to -8 :

$$
2|E(K)|+2|E(K)|-4(2+|E(K)|)=-8 .
$$

Define a "special" vertex to be a 3 -vertex that does not lie on the boundary of a $5^{+}$-face. Now we devise a set of rules for redistributing charge among the elements of $K$ so that the charge on every element becomes positive while the total charge is preserved. The rules for discharging are: 
Rule 1: Each $5^{+}$-face gives a charge of 1 to each 3-vertex on its boundary.

Rule 2: Each 5-vertex gives $1 / 3$ charge to every incident triangle.

Rule 3: Each 6 -vertex gives $1 / 3$ charge to every adjacent special vertex.

Rule 4 (a-c):

(a) Each 6 -vertex gives $2 / 3$ charge to every incident $(4,5,6)$-triangle.

(b) Each 6 -vertex gives $1 / 2$ charge to every incident $(4,6,6)$-triangle.

(c) Each 6-vertex gives $1 / 3$ charge to every incident $\left(5^{+}, 5^{+}, 6\right)$-triangle.

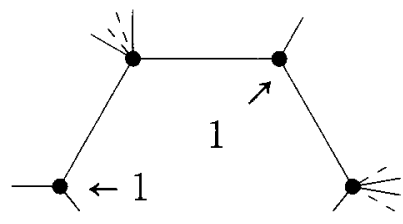

Rule 1

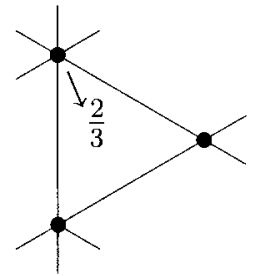

Rule $4 \mathrm{a}$

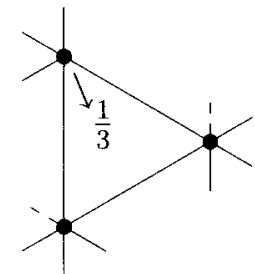

Rule 2

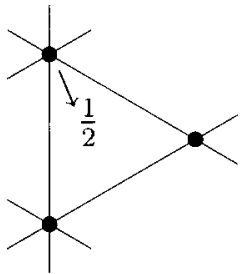

Rule $4 b$

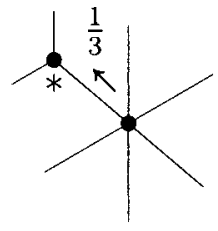

Rule 3

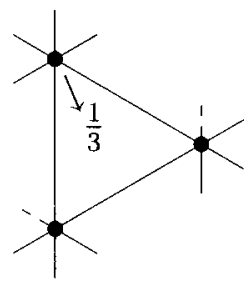

Rule $4 \mathrm{c}$

FIGURE 1.6: A visual description of the Discharging Rules.

Now we calculate the resulting charge on each type of element in $K$.

- A triangle originally receives a charge of -1 . Due to Lemmas 3,4 , and 5 , it must be either a $\left(4,5^{+}, 6\right)$-triangle or a $\left(5^{+}, 5^{+}, 5^{+}\right)$-triangle. $\mathrm{A}(4,5,6)$ triangle receives $1 / 3$ charge from the 5 -vertex and $2 / 3$ charge from the 6 vertex. A $(4,6,6)$-triangle receives $1 / 2$ charge from each of the 6 -vertices. A $\left(5^{+}, 5^{+}, 5^{+}\right)$-triangle receives $1 / 3$ charge from each of its boundary vertices. In each case, the resulting charge is 0 . 
- A 4-face originally receives zero charge. The discharging rules do not take charge away from it, so it remains zero.

- A 5 -face originally receives a charge of 1 . By Rule 1 , it loses 1 charge for every 3 -vertex on its boundary. By Lemma 7 , there can be no more than one of those. Since the 5-face can only lose 1 charge this way, it ends up with a charge of 0 or 1 .

- A 6-face originally receives a charge of 2. By Rule 1, it loses 1 charge for every 3 -vertex on its boundary. By Lemma 8, there can be no more than two of those. Therefore, the greatest charge a 6 -face can lose is 2 , so the resulting charge is at least 0 .

- A $7^{+}$-face $f$ originally receives a charge of $\operatorname{deg}(f)-4$. By Lemma 2, no more than $\left\lfloor\frac{\operatorname{deg}(f)}{2}\right\rfloor 3$-vertices can lie on its boundary. The face gives a charge of 1 to each of these, so the resulting charge is at least $\operatorname{deg}(f)-4-\lfloor\operatorname{deg}(f) / 2\rfloor=$ $\lceil\operatorname{deg}(f) / 2\rceil-4 \geq 0$.

- Neither a 1- or 2-vertex cannot appear in $K$, by Lemma 1 .

- A 3-vertex originally receives a charge of -1 . Unless it is special, it receives 1 charge from at least one incident $5^{+}$-face by Rule 1 . If it is special, it receives $1 / 3$ charge from each of its three neighbor 6 -vertices by Rule 3 . In either case, the result is nonnegative.

- A 4-vertex originally receives zero charge. The discharging rules do not take charge away from it, so it stays zero.

- A 5-vertex originally receives a charge of 1 . By Rule 2 , it loses $1 / 3$ charge to each incident triangle. Therefore, if it has no more than three incident triangles, the resulting charge is nonnegative. There are at most $v_{5}^{4} 5$-vertices 
with four incident triangles, each of which ends up with a charge of $-1 / 3$, and there are at most $v_{5}^{5^{+}} 5$-vertices with five incident triangles (which is the maximum), each of which ends up with a charge of $-2 / 3$. Therefore, the total charge on all 5 -vertices is at least $-(1 / 3) v_{5}^{4}-(2 / 3) v_{5}^{5^{+}}$.

- A $7^{+}$-vertex cannot appear in $K$, because $\Delta(K) \leq 6$.

- A 6-vertex $v$ originally receives a charge of 2. We will consider several cases, based on the number of triangles incident to $v$.

If $v$ has no incident triangles, it loses nothing to Rule 4 , but will still lose $1 / 3$ charge to each special neighbor by Rule 3 . The resulting charge on $v$ will be at least $2-6(1 / 3)=2-2=0$.

If $v$ has only one incident triangle, it loses up to $2 / 3$ charge to that triangle by Rule 4 . Vertices on a triangle cannot be special, so $v$ has at most 4 special neighbors. Its resulting charge will be at least $2-2 / 3-4(1 / 3)=0$.

If $v$ has two incident triangles, it loses up to $2 / 3$ charge to each of them by Rule 4. If those triangles share an edge, then three neighbors of $v$ lie on those triangles, and at most two of the others can be special, by (1.3). If they do not share an edge, then four neighbors of $v$ lie on those triangles, leaving only two neighbors which are potentially special. Since $v$ has at most two special neighbors and loses $1 / 3$ charge to each by Rule 3 , its resulting charge is at least $2-2(2 / 3)-2(1 / 3)=2-2=0$.

If $v$ has three incident triangles and no special neighbors, then it only loses up to $2 / 3$ charge to each incident triangle, so its remaining charge is at least $2-3(2 / 3)=0$. Alternatively, suppose $v$ has at least one special neighbor. This implies at least two of the three triangles share an edge. 

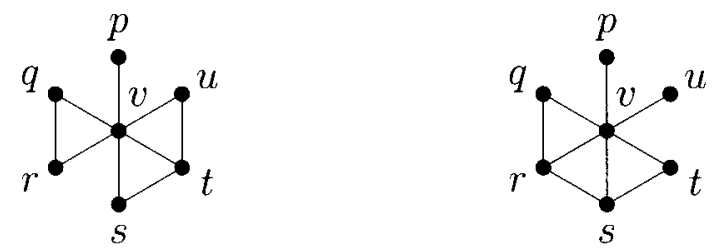

FIGURE 1.7: Two configurations in which a 6-vertex has three incident triangles and one special neighbor.

Case 1: One incident triangle shares no edge with the others, and $v$ has only one neighbor that does not lie on a triangle incident to $v$, as shown on the left in Figure 1.7. Let $p$ be the special vertex; then, by (1.3) and Lemma 10 , neither $v q r$ nor $v t u$ is a $(4,5,6)$-triangle. Vertex $v$ will give $1 / 3$ charge to $p$, up to $1 / 2$ charge to each of $v q r$ and $v t u$, and up to $2 / 3$ charge to $v s t$. The resulting charge on $v$ is at least $2-1 / 3-2(1 / 2)-2 / 3=0$.

Case 2: Every incident triangle shares an edge with another, and $v$ has two neighbors that do not lie on a triangle incident to $v$, as shown on the right in Figure 1.7. Let $p$ be the special vertex. Then, by (1.3) and Lemma 10 , vqr cannot be a $(4,5,6)$-triangle, and it gets at most $1 / 2$ charge from $v$. If $\operatorname{deg}(q)=4$, then $\operatorname{deg}(r)=6$, so vrs must get at most $1 / 2$ charge as well. If $\operatorname{deg}(r)=4$, then vrs and vst cannot both be $(4,5,6)$-triangles, by Lemma 9. If $\operatorname{deg}(q), \operatorname{deg}(r) \geq 5$, then $v$ gives only $1 / 3$ charge to $v q r$. In every case, $v$ does not give more than $5 / 3$ charge to its triangles, so the resulting charge on $v$ is at least $2-1 / 3-5 / 3=0$.

If $v$ has four incident triangles and a special neighbor $p$, the incident triangles nearest $p$ cannot be $(4,5,6)$-triangles, by $(1.3)$ and Lemma 10 . They will each receive at most $1 / 2$ charge from $v$. The other triangles may receive up to $2 / 3$ charge each, leaving $v$ with at charge of at least $2-1 / 3-2(1 / 2+2 / 3)=-2 / 3$. If no special neighbor is present, $v$ can lose up to $2 / 3$ charge to each triangle, 
leaving it with a charge of at least $2-4(2 / 3)=-2 / 3$. A 6 -vertex that lies on four distinct 3-cycles can potentially have four incident triangles, so the total amount of charge on all such vertices will be at least $-(2 / 3) v_{6}^{4}$.

If $v$ has five incident triangles, then every neighbor lies on a triangle and cannot be special. It cannot be adjacent to three incident $(4,5,6)$-triangles in a row, as this would imply the existence of the configuration banned by Lemma 9. Therefore, at most four incident faces are $(4,5,6)$-triangles. If there are four, then the third in the sequence cannot be one, and in fact must be a (5,5,6)-triangle, so after discharging, $v$ is left with a charge of $2-4(2 / 3)-1 / 3)=-1$. If fewer than four triangles incident to $v$ are $(4,5,6)$, then it is still left with at least $2-3(2 / 3)-2(1 / 2)=-1$ charge. A 6 -vertex that lies on five distinct 3-cycles could lie on up to five incident triangles, so the total amount of charge on all such vertices will be at least $-v_{6}^{5}$.

If $v$ has six incident triangles, then at most four of them are $(4,5,6)$-triangles, because Lemma 9 prevents three from occuring in a sequence around $v$. If there are exactly four, then the other two triangles must be $(5,5,6)$, so after discharging, $v$ is left with a charge of $2-4(2 / 3)-2(1 / 3)=-4 / 3$. If $v$ has two or fewer incident $(4,5,6)$-triangles, then it is left with at least $2-2(2 / 3)-4(1 / 2)=$ $-4 / 3$ charge. Suppose, instead, that $v$ has three incident $(4,5,6)$-triangles. To avoid the configurations banned by Lemmas 4 and $9, v$ must have exactly two neighbors of degree 4, diametrically opposite one another. One of those lies on two $(4,5,6)$-triangles with $v$, and the other must lie on a $(4,5,6)$-triangle and a (4,6,6)-triangle with $v$. Having determined what sort of triangles surround $v$, it must be left with a charge of exactly $2-3(2 / 3)-2(1 / 3)-1 / 2=-7 / 6$. A 6 -vertex that lies on six or more distinct 3-cycles can potentially lie on up to six incident triangles, so the total amount of charge on all such vertices will be at least $-(4 / 3) v_{6}^{6^{+}}$. 


\subsection{Conclusion}

After the discharging, almost every type of element has nonnegative charge. The only ones that may have negative charge are the $5^{+}$-vertices that lie on more than three 3-cycles. But we have a lower bound on the total charge these vertices can have collectively:

$$
\begin{gathered}
-(1 / 3) v_{5}^{4}-(2 / 3) v_{5}^{5^{+}}-(2 / 3) v_{6}^{4}-v_{6}^{5}-(4 / 3) v_{6}^{6^{+}} \\
=-1 / 3\left(v_{5}^{4}+2\left(v_{5}^{5^{+}}+v_{6}^{4}\right)+3 v_{6}^{5}+4 v_{6}^{6^{+}}\right)
\end{gathered}
$$

Since $K$ satisfies (1.2), the quantity being multiplied by $-1 / 3$ in (3) is less than 24 , which means the total charge is greater than -8 . Since that contradicts (1.4), we conclude that $K$ is not a possible graph. As $K$ was chosen arbitrarily to be one with a minimal number of edges in $\Gamma$, no such graph is possible, so $\Gamma$ must be empty. Then there is no counterexample to the Theorem, and the proof is complete.

\subsection{The Problem with Triangles}

An easy corollary can be derived from Theorem 1. It is essentially a weaker, but more accessible, version:

Corollary to Theorem 1 . If $G$ is a planar graph wherein no vertex of degree $5^{+}$lies on more than three distinct 3-cycles, then $\chi^{T}(G) \leq$ $\Delta(G)+2$.

Proof. The given information indicates that $v_{5}^{4}=v_{5}^{5^{+}}=v_{6}^{4}=v_{6}^{5}=v_{6}^{6^{+}}=0$, so equation (1.2) is satisfied, so by Theorem $1, \chi^{T}(G) \leq \Delta(G)+2$.

It makes sense to try to modify this theorem, or its corollary, to pertain to triangles instead of 3-cycles. Such a modification would have several advantages 
over Theorem 1. First, it would be easier to visualize. Second, the result would apply to a larger family of graphs, because it would apply to graphs in which vertices are part of few triangles but many 3 -cycles. But the statement of the theorem for triangles is slightly complicated by the choice that exists for planar embeddings of the graph. Triangles are elements of a particular embedding, not elements of an abstract collection of vertices and edges. The modified theorem would have to apply to either graphs for which some planar embedding has a limited number of triangles adjacent to every high-degree vertex, or graphs for which every planar embedding has this property. Let's consider both ways that such a modification of this corollary might be stated:

Conjecture 1: Suppose $G$ is a planar graph, and some planar embedding of $G$ has the property that no $5^{+}$-vertex is incident to more than three triangles. Then $\chi^{T}(G) \leq \Delta(G)+2$.

Conjecture 2: Suppose $G$ is a planar graph, and every planar embedding of $G$ has the property that no $5^{+}$-vertex is incident to more than three triangles. Then $\chi^{T}(G) \leq \Delta(G)+2$.

To prove either one of these in the same way that Theorem 1 was proven, we could choose a minimum counterexample $K$, find a set of reducible configurations for $K$, and then use discharging to prove that one of those configurations must exist in $K$. In every lemma used in that proof to explain why a configuration was reducible, elements were deleted from $K$ to obtain a proper subgraph that was total-8-colorable because it satisfied (1.2). But in a proof adapted for Conjecture 1 or 2, a major problem arises when we try to delete elements: Triangles can be created. The deletion of a single vertex or edge can potentially produce a subgraph that no longer satisfies the conditions of the conjecture, so we would have no guarantee that such a subgraph would be total-8-colorable. 

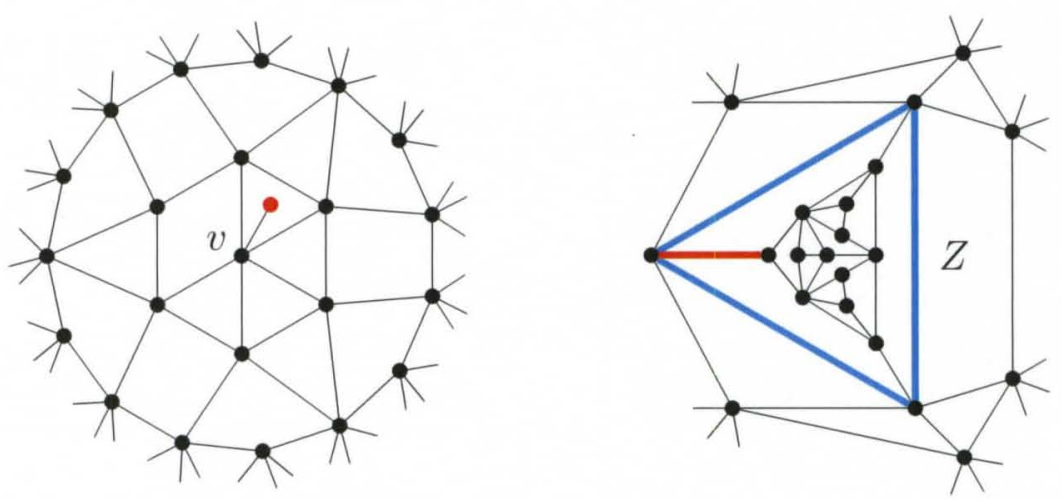

FIGURE 1.8: $K$, a minimum counterexample to Conjecture 1 (or 2), might satisfy the conditions for that Conjecture and still contain a configuration to one shown above. However, this could imply that a subgraph of $K$ might not satisfy the same conditions.

For example, suppose we chose a graph $K$ as a minimum counterexample to Conjecture 1 . We cannot exclude the possibility that $K$ contains the configuration on the left in Figure 1.8, which can be embedded into the plane as shown so that the central 6-vertex is only incident to three triangles. The proof of Lemma 1 depends on the deletion of a leaf producing a subgraph for which "some planar embedding has the property that no $5^{+}$-vertex is incident to more than three triangles." But the subgraph produced by the deletion of $v$ 's leaf neighbor will not satisfy that property, as it leaves $v$ incident to four triangles no matter what planar embedding is considered. Lemma 1 fails to prove that the 1 -vertex is reducible, so the proof cannot work the same way.

Suppose, instead, that we were trying to prove the less powerful Conjecture 2 , and that we chose $K$ as a minimum counterexample. This time, we can assume the aforementioned configuration is not in $K$, because it can be embedded with the leaf in the 4-cycle so that $v$ is incident to too many triangles. However, $K$ could contain the configuration on the right in the same figure, in which, for every planar embedding, no vertex is incident to more than 3 triangles. The proof of Lemma 2 (as well as most of the other lemmas) depends on the deletion of an 
edge, producing a subgraph for which "every planar embedding has the property that no $5^{+}$-vertex is incident to more than three triangles." But if a subgraph were produced by the deletion of the red $(3,5)$-edge, that subgraph could be redrawn in such a way that the configuration inside the blue 3-cycle is embedded in face $Z$ instead, and that turns the blue 3-cycle into a triangle. In that embedding, two of the vertices around that triangle are incident to too many triangles, so the conjecture's condition is not satisfied by that subgraph. Lemma 2 , as well as some other lemmas, will fail, so the proof cannot work the same way. 


\section{CHAPTER 2 TOTAL INDEPENDENT SETS AND THE TOTAL INDEPENDENCE NUMBER}

\subsection{Independence for all Elements}

The application of independence to both vertices and edges of a graph is as old as total coloring. Let's recall that a total independent set is a set of elements in $G$, none of which are incident or adjacent. All independent sets and matchings count as total independent sets, but only a total independent set can include both vertices and edges. When we total-color a graph, a total independent set could constitute all elements of a single color, so essentially, a total coloring is a partitioning of $V \cup E$ into total independent sets.

The cardinality of the largest possible total independent set in a graph $G$ is called the total independence number of $G$, or $\alpha^{T}(G)$, to keep it consistent with the notation for total chromatic number. A maximum total independent set in $G$ must have size $\alpha^{T}(G)$. This parameter is also useful because it is an upper bound on the number of elements in $G$ that can share a color. In this respect, it gives us a lower bound on the total chromatic number of $G$, because the number of colored elements cannot be more than the number of colors times the number of elements that can be assigned the same color:

$$
\chi^{T}(G) \geq \frac{|V(G)|+|E(G)|}{\alpha^{T}(G)} .
$$




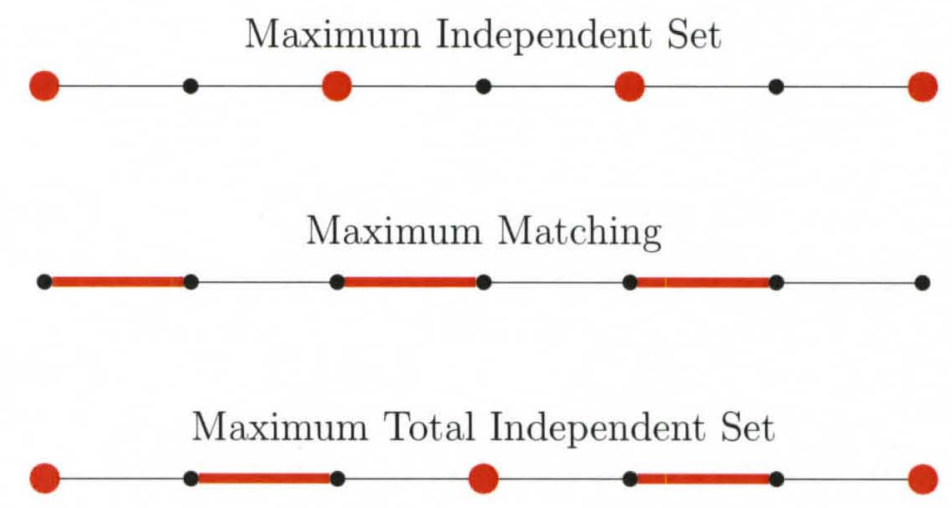

FIGURE 2.1: Three graphs, all paths on seven vertices, in which a maximum independent set, a maximum matching, and a maximum total independent set are demonstrated.

In Figure 2.1, maximum independent sets of each type (vertex, edge, and total) are shown in the path $P_{7}$ to demonstrate the differences between the concepts. We could reason that this path could not contain more than 5 elements in a total independent set, and that the total independence number of $P_{7}$ is 5 . Similar reasoning could find the total independence number in general for all paths: We could partition the elements of the path $P_{n}=v_{1} v_{2} \ldots v_{n}$ into subsets each containing three elements, as follows: $H_{1}=\left\{v_{1}, v_{1} v_{2}, v_{2}\right\}, H_{2}=\left\{v_{2} v_{3}, v_{3}, v_{3} v_{4}\right\}$, and so on. The last subset might include fewer than 3 elements. Since there are $2 n-1$ elements in total, including edges, there would be $\left[\frac{2 n-1}{3}\right\rceil$ such sets. Every set $H_{i}$ is dependent, because the elements in each set are close enough that they cannot share colors. Then every total independent set $T$ of $P_{n}$ can have at most one element in each set $H_{i}$, so $\alpha^{T}\left(P_{n}\right) \leq\left\lceil\frac{2 n-1}{3}\right\rceil$. This is also a lower bound, because $T$ can be chosen using the first element of each set $H_{i}$ without a conflict. Then $\alpha^{T}\left(P_{n}\right)=\left\lceil\frac{2 n-1}{3}\right\rceil$. The following theorem, which pertains to the total independence number of a path, tells us a little bit more about these total independent sets. 
Theorem 2. For each $n \geq 2$, if $P_{n}$ is the path from $v_{1}$ to $v_{n}$, and $\alpha^{T}=\alpha^{T}\left(P_{n}\right)$, then $\alpha^{T}=\left\lceil\frac{2 n-1}{3}\right\rceil$ and:

- If $n \equiv 1(\bmod 3)$, then a total independent set of size $\alpha^{T}$ in $P_{n}$ must include $v_{n}$.

- If $n \equiv 2(\bmod 3)$, then a total independent set of size $\alpha^{T}$ in $P_{n}$ does not need to include either $v_{n} v_{n-1}$ nor $v_{n}$.

- If $n \equiv 0(\bmod 3)$, then a total independent set of size $\alpha^{T}$ in $P_{n}$ need not include $v_{n}$, but must include either that or $v_{n} v_{n-1}$.

Proof. When $n=2$, the graph is just a pair of adjacent vertices, only one of which can be in a total independent set. Since the choice of the element does not matter, the set can include only $v_{1}$ and leave both $v_{1} v_{2}$ and $v_{2}$ unchosen. Also, $\left\lceil\frac{2 n-1}{3}\right\rceil=$ $\left\lceil\frac{3}{3}\right\rceil=1$. This is all consistent with the theorem.

The theorem for all $n>2$ is proved by induction on $n$. Suppose it is true for $n=k$, and consider a total independent set $T$ within the path $P_{k+1}$. Depending on the modularity of $k$, choose the appropriate case below.

Case $1: k \equiv 1(\bmod 3)$. If $T$ includes $\alpha^{T}\left(P_{k}\right)$ elements in the subset $P_{k}$, that amounts to a total independent set of maximum size in that subset, so by the theorem, it must include $v_{k}$; then neither $v_{k+1}$ nor $v_{k} v_{k+1}$ can be in $T$, so $|T|=$ $\alpha^{T}\left(P_{k}\right)$. Otherwise - if $T$ includes fewer than $\alpha^{T}\left(P_{k}\right)$ elements in the subset $P_{k}-$ it is possible that either $v_{k+1}$ or $v_{k} v_{k+1}$ might be in $T$, but not both, so the total number of elements in $T$ must be less than $\alpha^{T}\left(P_{k}\right)+1$. Either way, the greatest $|T|$ can be is $\alpha^{T}\left(P_{k}\right)$, so $\alpha^{T}\left(P_{k+1}\right)=\alpha^{T}\left(P_{k}\right)=\left\lceil\frac{2 k-1}{3}\right\rceil=\left\lceil\frac{(k-1)+(k-1)+1}{3}\right\rceil=2\left(\frac{k-1}{3}\right)+\left\lceil\frac{1}{3}\right\rceil$ $=2\left(\frac{k-1}{3}\right)+\left\lceil\frac{3}{3}\right\rceil=\left\lceil\frac{(k-1)+(k-1)+3}{3}\right\rceil=\left\lceil\frac{2(k+1)-1}{3}\right\rceil$. This satisfies the first part of the theorem. We can also see that it is possible to avoid including $v_{k+1}$ and $v_{k} v_{k+1}$ by keeping elements the entire total independent set inside the subset $P_{k}$, and this 
satisfies the second part of the theorem for $n=k+1$. Therefore, the theorem is true when $n=k+1$.

Case 2: $\quad k \equiv 2(\bmod 3)$. Then by the theorem, it is possible for $T$ to include $\alpha^{T}\left(P_{k}\right)$ elements in the subset $P_{k}$ in such a way that neither $v_{k}$ nor $v_{k} v_{k-1}$ is chosen. Do this, then add either $v_{k} v_{k+1}$ or $v_{k+1}$ to $T$, to make $|T|=\alpha^{T}\left(P_{k}\right)+1$. T cannot be made larger than this, since only $\alpha^{T}\left(P_{k}\right)$ elements can be in the subset, and only one can be in $\left\{v_{k+1}, v_{k} v_{k+1}\right\}$. Therefore, $\alpha^{T}\left(P_{k+1}\right)=\alpha^{T}\left(P_{k}\right)+1=\left\lceil\frac{2 k-1}{3}\right\rceil+1=$ $\left\lceil\frac{(k-2)+(k-2)+3}{3}\right\rceil+1=2\left(\frac{k-2}{3}\right)+\left\lceil\frac{3}{3}\right\rceil+1=2\left(\frac{k-2}{3}\right)+\left\lceil\frac{5}{3}\right\rceil=\left\lceil\frac{(k-2)+(k-2)+5}{3}\right\rceil=\left\lceil\frac{2(k+1)-1}{3}\right\rceil$. This satisfies the first part of the theorem. Also observe that either $v_{k+1}$ or $v_{k} v_{k+1}$ must be in a total independent set of this size, and it does not matter which, and this satisfies the second part of the theorem for $n=k+1$. Therefore, the theorem is true when $n=k+1$.

Case 3: $k \equiv 0(\bmod 3)$. If $T$ includes $\alpha^{T}\left(P_{k}\right)$ elements in the subset $P_{k}$, then by the theorem, it must include either $v_{k}$ or $v_{k} v_{k-1}$; this prevents $v_{k} v_{k+1}$ from being in $T$, but it is possible to choose the set so that $v_{k}$ is not in $T$, and this allows $T$ to include $v_{k+1}$, so that $|T|=\alpha^{T}\left(P_{k}\right)+1$. Otherwise - if $T$ includes fewer than $\alpha^{T}\left(P_{k}\right)$ elements in the subset $P_{k}$ - then the most it can have is $\alpha^{T}\left(P_{k}\right)-1$ elements from that subset and one from $\left\{v_{k}, v_{k} v_{k+1}\right\}$. Among these choices, the greatest $|T|$ can be is $\alpha^{T}\left(P_{k}\right)+1=\left\lceil\frac{2 k-1}{3}\right\rceil+1=2\left(\frac{k}{3}\right)+\left\lceil\frac{-1}{3}\right\rceil+1=2\left(\frac{k}{3}\right)+\left\lceil\frac{1}{3}\right\rceil=\left\lceil\frac{2 k+1}{3}\right\rceil=\left\lceil\frac{2(k+1)-1}{3}\right\rceil$. This satisfies the first part of the theorem. Also observe that the only way $|T|$ can reach this size is if it includes $v_{k+1}$, and this satisfies the second part of the theorem for $n=k+1$. Therefore, the theorem is true when $n=k+1$.

\subsection{Total Independence Number of Other Common Graphs}

In this section the value of $\alpha^{T}$ will be found for some other common graphs, including cycles, complete graphs, and complete bipartite graphs. 
Theorem 3. For each integer $n \geq 3, \alpha^{T}\left(C_{n}\right)=\left\lfloor\frac{2 n}{3}\right]$.

Proof. Denote the set of all vertices and edges as $X=V\left(C_{n}\right) \cup E\left(C_{n}\right)$. Label the vertices $v_{1}, v_{2}, \ldots, v_{n}$, so that $v_{1}$ is adjacent to $v_{n}$, and $v_{k}$ is adjacent to $v_{k-1}$ for every integer $k$ from 2 to $n$.

Suppose $T \subset X$ is a total independent set in $C_{n}$. Each vertex in $T$ corresponds to two edges (the ones incident to it) that cannot be in $T$, and no two vertices can correspond to the same edge in this way, because no edge has two endvertices in $T$. Each edge in $T$ corresponds to two vertices (its endvertices) that cannot be in $T$, and no two edges can correspond to the same vertex in this way, because no vertex has two incident edges in $T$. Therefore, for every element in $T$, there are at least two elements in $X \backslash T$, so $|X \backslash T| \geq 2|T|$. Then

$$
\begin{gathered}
|X|=|T|+|X \backslash T| \geq|T|+2|T|=3|T|, \\
\frac{|X|}{3} \geq|T| .
\end{gathered}
$$

With this upper bound on the size of a total independent set, and the fact that $|X|=2 n$, we can see that $\alpha^{T}\left(C_{n}\right) \leq \frac{2 n}{3}$. The total independence number of $C_{n}$ is an integer, so this is the same as saying $\alpha^{T}\left(C_{n}\right) \leq\left\lfloor\frac{2 n}{3}\right\rfloor$. To prove equality, it is sufficient to show that a set $T$ exists of this size in $C_{n}$. In each case below, a total independent set is created of size $\left[\frac{2 n}{3}\right\rfloor$. Since we have shown this to be an upper bound, this must be the total independence number. 

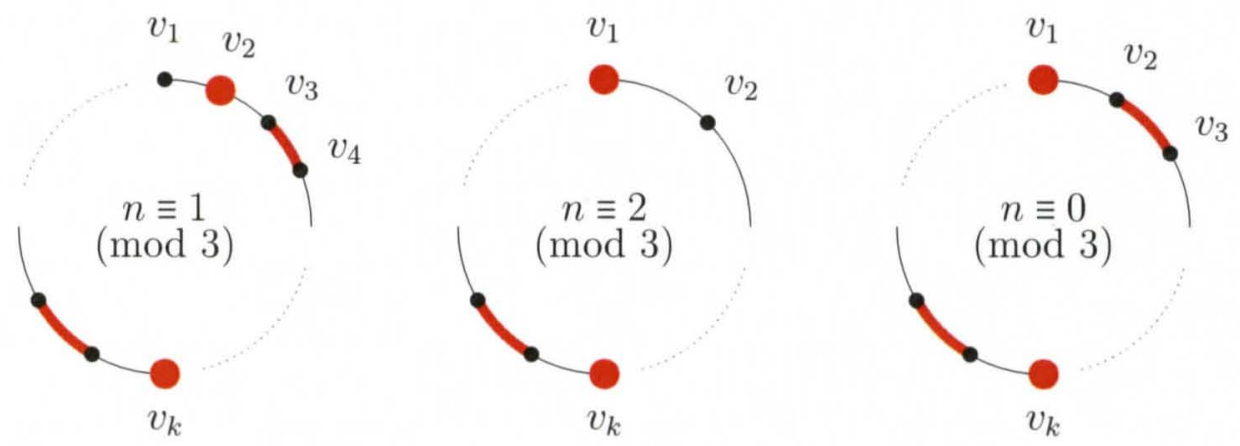

FIGURE 2.2: Choice of a maximum total independent set in $C_{n}$, depending on $n(\bmod 3)$.

Case 1: $n \equiv 1(\bmod 3)$.

Let $T=\left\{v_{k}, v_{k+1} v_{k+2} \mid 1 \leq k<n\right.$ and $\left.k \equiv 2(\bmod 3)\right\}$.

Then vertices in $T$ are mutually non-adjacent, edges in $T$ are mutually nonincident, and no vertex in $T$ is an endvertex of an edge in $T$. The number of elements in $T$ is twice the number of integers $k$ satisfying these conditions (which is $\left.\frac{n-1}{3}\right)$, so $\left\lfloor\frac{2 n}{3}\right\rfloor=\left\lfloor\frac{n-1}{3}+\frac{n-1}{3}+\frac{2}{3}\right\rfloor=\frac{n-1}{3}+\frac{n-1}{3}+0=2\left(\frac{n-1}{3}\right)=|T|$.

Case 2: $n \equiv 2(\bmod 3)$.

Let $T=\left\{v_{1}\right\} \cup\left\{v_{k}, v_{k+1} v_{k+2} \mid 1 \leq k<n\right.$ and $\left.k \equiv 0(\bmod 3)\right\}$.

Then vertices in $T$ are mutually non-adjacent, edges in $T$ are mutually nonincident, and no vertex in $T$ is an endvertex of an edge in $T$. The number of elements in $T$ is one plus twice the number of integers $k$ satisfying these conditions (which is $\left.\frac{n-2}{3}\right)$, so $\left\lfloor\frac{2 n}{3}\right\rfloor=\left\lfloor\frac{n-2}{3}+\frac{n-2}{3}+\frac{4}{3}\right\rfloor=2\left(\frac{n-2}{3}\right)+1=|T|$.

Case 3: $n \equiv 0(\bmod 3)$.

Let $T=\left\{v_{k}, v_{k+1} v_{k+2} \mid 1 \leq k<n\right.$ and $\left.k \equiv 1(\bmod 3)\right\}$.

Then vertices in $T$ are mutually non-adjacent, edges in $T$ are mutually nonincident, and no vertex in $T$ is an endvertex of an edge in $T$. The number of elements in $T$ is twice the number of integers $k$ satisfying these conditions (which is $\left.\frac{n}{3}\right)$, so $\left\lfloor\frac{2 n}{3}\right\rfloor=\left\lfloor\frac{n}{3}+\frac{n}{3}\right\rfloor=2\left(\frac{n}{3}\right)=|T|$. 
Theorem 4. For each integer $n \geq 1, \alpha^{T}\left(K_{n}\right)=\left\lceil\frac{n}{2}\right\rceil$.

Proof. Suppose $T$ is a total independent set in $K_{n}$. Only one vertex of $K_{n}$ can be in $T$, because all the vertices are adjacent. If there are no vertices in $T$, then $T$ can only include edges, so its size is equal to the size of the largest matching in $K_{n}$, and that is $\nu\left(K_{n}\right)$, which is easily calculated to be $[n / 2\rfloor$. If $T$ does include a vertex $u$, then the only other elements possible for $T$ are edges that don't have $u$ as an endvertex: Namely, the edges of $K_{n}-u$, which is the same as the graph $K_{n-1}$. Then the size of $T$ will be one (the vertex) plus the size of the largest matching in $K_{n-1}$, and this is equal to $1+\left\lfloor^{(n-1)} / 2\right\rfloor=\left\lfloor^{(n+1)} / 2\right\rfloor=\lceil n / 2\rceil$. Whether or not $T$ contains a vertex, its maximum size is equal to $\lfloor n / 2\rfloor$.

Theorem 5. For every pair of integers $m, n \geq 1, \alpha^{T}\left(K_{m, n}\right)=\max \{m, n\}$.

Proof. Let $M$ and $N$ be the two independent vertex sets of $K_{m, n}$, such that $|M|=m$ and $|N|=n$. Suppose $T$ is a total independent set in $K_{m, n}$. Either $T \cap N$ or $T \cap M$ must be empty, because the inclusion of a vertex from each set would leave $T$ with a pair of adjacent vertices, which is impossible. If $T \cap N=\varnothing$, then $T$ can only include vertices in $M$ and edges that have an endvertex in $M$. Then $T$ contains at most one element for each vertex $M$ : either the vertex itself, or an edge incident to it: $|T| \leq m$. Similarly, if $T \cap M=\varnothing$, then $|T| \leq n$. Therefore, $|T| \leq \max \{m, n\}$, so $\alpha^{T} \leq \max \{m, n\}$. A total independent set equal to this number can be made by selecting all vertices in the larger of the two sets, so $\alpha^{T}=\max \{m, n\}$. 
Theorem 6. For each integer $n \geq 3, \alpha^{T}\left(S_{n}\right)=n$.

Proof. Let $T$ be a total independent set in the star. All elements are either adjacent or incident to the central vertex, so if that is in $T$, then nothing else can be, and $|T|=1$. If $T$ does not include the central vertex, then it contains at most one element for each leaf: either the leaf itself, or the edge to it from the central vertex. Thus, $|T| \leq n$. It is possible to make $|T|=n$ by selecting all leaves, so the total independence number is $n$.

\subsection{Total Independence Number of Full Binary Trees}

The family of trees is countably infinite, and to construct a formula for total independence number as a function of any given tree would involve too many parameters. However, if some restriction is made that greatly reduces the complexity of the trees in question and reduces the number of parameters to around, say, one, then it becomes more practical to make a formula for the total independence number. For example, restricting the maximum distance to 2 yields the family of stars, for which the total independence number was found in the previous section.

There are easily an infinitude of other families of trees whose members are the range of a function of a single integer, for example "five paths of length $n$ meeting at a common endpoint," or " $n$ paths of length five meeting at a common endpoint," etc. Many of these may be worthy of study at some point. However, the only other family of trees which will be studied in this chapter are the full binary trees, which are graphs that are useful in computer algorithms for searching and sorting, and for representing various heirarchies and tournaments. 
Theorem 7. For each integer $n \geq 1$, if $B_{n}$ is the full binary tree of height $n$ having root vertex $v$, then:

- If $n \equiv 1(\bmod 3)$, then $\alpha^{T}\left(B_{n}\right)=2 \alpha^{T}\left(B_{n-1}\right)$, and a total independent set of this size does not need to include either $v$ nor its incident edges.

- If $n \equiv 2(\bmod 3)$, then $\alpha^{T}\left(B_{n}\right)=2 \alpha^{T}\left(B_{n-1}\right)+1$, and a total independent set of this size need not include $v$, but must include either that or an edge incident to $v$.

- If $n \equiv 0(\bmod 3)$, then $\alpha^{T}\left(B_{n}\right)=2 \alpha^{T}\left(B_{n-1}\right)+1$, and a total independent set of this size must include $v$.

Proof. The theorem will be proved by induction on $n$. It is not difficult to see that $\alpha^{T}\left(B_{0}\right)=1$ and $\alpha^{T}\left(B_{1}\right)=2$. So, if $n=1$, the theorem is true because $\alpha^{T}\left(B_{n}\right)=2 \alpha^{T}\left(B_{n-1}\right)$, and a total independence set of size $\alpha^{T}\left(B_{1}\right)=2$ can be chosen that only includes the two leaves in $B_{1}$.

Suppose the theorem is true for $n=k$. Consider the graph $B_{k+1}$, and let $v$ be its root vertex. Observe that the two neighbors of $v$, which will be called $v_{G}$ and $v_{H}$, are the root vertices of two mutually disjoint $B_{k}$ subgraphs of $B_{k+1}$, which we can call $G$ and $H$, respectively. The theorem gives us some information about the largest total independent set that can exist in $B_{k}$, which will help us find the largest possible total independent set $T$ within $B_{k+1}$. Depending on the modularity of $k$, choose the appropriate case below.

Case 1: $\quad k \equiv 2(\bmod 3)$. Suppose $T$ contains $\alpha^{T}\left(B_{k}\right)$ elements from each of $G$ and $H$. Then, by the theorem for $n=k$, either $v_{G}$ or one of its incident edges (other than $v v_{G}$ ) must be in $T$, and either $v_{H}$ or one of its incident edges (other than $v v_{H}$ ) must also be in $T$. Therefore, neither $v v_{G}$ or $v v_{H}$ may be selected. It is possible to have selected independent elements in the subgraphs such that neither 
$v_{G}$ nor $v_{H}$ was selected, and if this is done, it is possible for $v$ to be in $T$, which makes $|T|=2 \alpha^{T}\left(B_{k}\right)+1$.

Suppose, instead, that $T$ contains fewer than $\alpha^{T}\left(B_{k}\right)$ elements from at least one of the subgraphs $G$ or $H$. Since there can be only one element from $\left\{v, v v_{G}, v v_{H}\right\}$ in $T$, we have $|T|<\alpha^{T}\left(B_{k}\right)+\alpha^{T}\left(B_{k}\right)+1$, so $|T| \leq 2 \alpha^{T}\left(B_{k}\right)$.

It follows that the upper bound for $|T|$ is $2 \alpha^{T}\left(B_{k}\right)+1$, and this number can be obtained if and only if $v \in T$. This satisfies the result of the theorem for $n=k+1 \equiv 0(\bmod 3)$.

Case 2: $\quad k \equiv 0(\bmod 3)$. Suppose $T$ contains $\alpha^{T}\left(B_{k}\right)$ elements from each of $G$ and $H$. Then, by the theorem for $n=k$, both $v_{G}$ and $v_{H}$ must be in $T$. This implies that no element from $\left\{v, v v_{G}, v v_{H}\right\}$ can be in $T$, so $|T|=2 \alpha^{T}\left(B_{k}\right)$.

Suppose, instead, that $T$ contains fewer than $\alpha^{T}\left(B_{k}\right)$ elements from at least one of the subgraphs $G$ or $H$. Since there can be only one element from $\left\{v, v v_{G}, v v_{H}\right\}$ in $T$, we have $|T|<\alpha^{T}\left(B_{k}\right)+\alpha^{T}\left(B_{k}\right)+1$, so $|T| \leq 2 \alpha^{T}\left(B_{k}\right)$.

Either way, the upper bound for $|T|$ is $2 \alpha^{T}\left(B_{k}\right)$. This number can be obtained by choosing $\alpha^{T}\left(B_{k}\right)$ elements from each of $G$ and $H$ and none from $\left\{v, v v_{G}, v v_{H}\right\}$. This satisfies the result of the theorem for $n=k+1 \equiv 1(\bmod 3)$.

Case 3: $k \equiv 1(\bmod 3)$. Suppose $T$ contains $\alpha^{T}\left(B_{k}\right)$ elements from each of $G$ and $H$. By the theorem for $n=k$, this can be done so that $T$ contains neither $v_{G}$ nor $v_{H}$ and none of the edges incident to those in $G$ or $H$. Then $T$ can also include one additional element from $\left\{v, v v_{G}, v v_{H}\right\}$. If none of those three are in $T$, we have $|T|=2 \alpha^{T}\left(B_{k}\right)$; but if one is included, then $|T|=2 \alpha^{T}\left(B_{k}\right)+1$.

Suppose, instead, that $T$ contains fewer than $\alpha^{T}\left(B_{k}\right)$ elements from at least one of the subgraphs $G$ or $H$. Since there can be only one element from $\left\{v, v v_{G}, v v_{H}\right\}$ in $T$, we have $|T|<\alpha^{T}\left(B_{k}\right)+\alpha^{T}\left(B_{k}\right)+1$, so $|T| \leq 2 \alpha^{T}\left(B_{k}\right)$.

It follows that the upper bound for $|T|$ is $2 \alpha^{T}\left(B_{k}\right)+1$. A set $T$ of this 
size can be constructed using $\alpha^{T}\left(B_{k}\right)$ elements from each set $G$ and $H$, plus one element from $\left\{v, v v_{G}, v v_{H}\right\}$, which does not have to be $v$. This satisfies the result of the theorem for $n=k+1 \equiv 2(\bmod 3)$.

With Theorem 7 established, we have a recurrence relation for $\alpha^{T}\left(B_{n}\right)$ :

$$
\begin{gathered}
\alpha^{T}\left(B_{0}\right)=1, \text { and } \\
\alpha^{T}\left(B_{n}\right)= \begin{cases}2 \alpha^{T}\left(B_{n-1}\right) & \text { if } n \equiv 1(\bmod 3), \\
2 \alpha^{T}\left(B_{n-1}\right)+1 & \text { otherwise. }\end{cases}
\end{gathered}
$$

Taking the first few values, starting with $\alpha^{T}\left(B_{0}\right)$, we obtain the sequence $1,2,5,11,22,45,91$, etc. Now a closed formula will be found for $\alpha^{T}\left(B_{n}\right)$. Define $a_{n}=\alpha^{T}\left(B_{n}\right)$ for each integer $n \geq 0$.

For $n \equiv 1(\bmod 3), a_{n}=2 a_{n-1}=2\left(2 a_{n-2}+1\right)=4\left(2 a_{n-2}+1\right)+2=8 a_{n-3}+6$;

solving for the homogeneous part, we obtain the general solution

$$
a_{n}=\lambda 2^{n}+c .
$$

Using the initial conditions $a_{1}=2$ and $a_{4}=22$, we get the system:

$$
\left\{\begin{array}{l}
2=2 \lambda+c \\
27=16 \lambda+c
\end{array} \quad \text { with the solutions } \lambda=\frac{10}{7}, c=\frac{-6}{7} .\right.
$$

This yields the particular solution $a_{n}=\frac{10}{7} 2^{n}-\frac{6}{7}=\left\lfloor\frac{10}{7} 2^{n}\right\rfloor$.

$$
\text { For } n \equiv 2(\bmod 3), a_{n}=2 a_{n-1}+1=2\left(2 a_{n-2}\right)+1=4\left(2 a_{n-2}+1\right)+1=8 a_{n-2}+5 \text {; }
$$

solving for the homogeneous part, we obtain the same general solution, and using $a_{2}=5$ and $a_{5}=45$, we get the system:

$$
\left\{\begin{array}{l}
5=4 \lambda+c \\
45=32 \lambda+c
\end{array} \quad \text { with the solutions } \lambda=\frac{10}{7}, c=\frac{-5}{7} .\right.
$$

This yields the particular solution $a_{n}=\frac{10}{7} 2^{n}-\frac{5}{7}=\left\lfloor\frac{10}{7} 2^{n}\right\rfloor$. 
For $n \equiv 0(\bmod 3), a_{n}=2 a_{n-1}+1=2\left(2 a_{n-2}+1\right)+1=4\left(2 a_{n-2}\right)+3=8 a_{n-2}+3$;

solving for the homogeneous part, we obtain the same general solution, and using $a_{0}=1$ and $a_{3}=11$, we get the system:

$$
\left\{\begin{array}{l}
1=\lambda+c \\
11=8 \lambda+c
\end{array} \quad \text { with the solutions } \lambda=\frac{10}{7}, c=\frac{-3}{7} .\right.
$$

This yields the particular solution $a_{n}=\frac{10}{7} 2^{n}-\frac{3}{7}=\left\lfloor\frac{10}{7} 2^{n}\right\rfloor$.

Therefore, $a_{n}=\alpha^{T}\left(B_{n}\right)=\left\lfloor\frac{10}{7} 2^{n}\right\rfloor$ for each $n \geq 0$.

\subsection{Total Independence and Edge Domination}

An interesting relationship exists between total independent sets and edge dominating sets, which enables us to calculate $\alpha^{T}$ based on other parameters of the graph: The edges in a maximum total independent set correspond to a minimum independent edge dominating set (a minimum maximal matching). This was first discovered by Yannakakis and Gavril [28], and their result is paraphrased here.

Theorem 8. (M. Yannakakis and F. Gavril, 1980)

For every graph $G$ with $n$ vertices, total independence number $\alpha^{T}$, and independent edge domination number $\gamma_{i}^{\prime}$,

$$
\alpha^{T}+\gamma_{i}^{\prime}=n
$$

Proof. Let $G$ be a graph with $n$ vertices. Let $M$ be a minimum independent edge dominating set in $G$; this will have cardinality $\gamma_{i}^{\prime}$. Let $W$ be the set of vertices that are not endvertices of an edge in $M . W$ must be an independent set, because the adjacency of a pair of vertices in $W$ would imply that the edge connecting them is neither in $M$ nor incident to an edge in $M$, and that would contradict the definition of $M$. Then $T=M \cup W$ must be a total independent set. 
Since every vertex is either in $W$ or is an endvertex of an edge in $M$, the number of vertices $n$ is equal to $|W|+2|M|$. Then:

$$
\begin{gathered}
\alpha^{T}(G) \geq|T|=|M|+|W|=|M|+(n-2|M|)=n-|M|=n-\gamma_{i}^{\prime} \\
\therefore n \leq \alpha^{T}+\gamma_{i}^{\prime} .
\end{gathered}
$$

Now suppose $T^{\prime}$ is total independent set in $G$ such that $\left|T^{\prime}\right|=\alpha^{T}(G)$. For every vertex $v \in V(G)$ that is not in $T^{\prime}$ and is not an endvertex of an edge in $T^{\prime}$, $v$ must have a neighbor $u$ in $T^{\prime}$ (otherwise $T^{\prime}+v$ would constitute a larger total independent set, which is impossible); remove $u$ from $T^{\prime}$ and add the edge $u v$ to $T^{\prime}$ instead. Repeat this process until every vertex in $V(G)$ is either an element of $T^{\prime}$ or is an endvertex of an edge in $T^{\prime}$.

Let $M^{\prime}$ and $W^{\prime}$ be the edges and vertices of $T^{\prime}$, respectively. Note that every edge $e \in E(G)$ must have at least one endvertex that is not in $T^{\prime}$, which means it must be the endvertex of an edge in $T^{\prime}$, which means it is an endvertex of an edge in $M^{\prime}$ (and might even be in $M^{\prime}$ ). This makes $M^{\prime}$ an edge dominating set in $G$, and implies $\left|M^{\prime}\right| \geq \gamma_{i}^{\prime}$.

Using the same argument as before, the number of vertices $n$ must be equal to $\left|W^{\prime}\right|+2\left|M^{\prime}\right|$, so:

$$
\alpha^{T}(G)=\left|T^{\prime}\right|=\left|M^{\prime}\right|+\left|W^{\prime}\right|=\left|M^{\prime}\right|+\left(n-2\left|M^{\prime}\right|\right)=n-\left|M^{\prime}\right| \leq n-\gamma_{i}^{\prime}
$$

$\therefore n \geq \alpha^{T}+\gamma_{i}^{\prime}$. With this and the earlier result, we obtain $n=\alpha^{T}+\gamma_{i}^{\prime}$.

An alternative proof is given by Zhang, Zhang, and Li in [30]. Using similar arguments, one can see that a maximum total independent set can be derived from a minimum independendent edge dominating set, and vice versa. This is important because results already exist for minimum independent edge dominating sets. It is useful in some cases to point out that a minimum independent edge dominating set is the same thing as a minimum maximal matching, as observed by Forcade in [6]. 
It is also worth noting that every minimum independendent edge dominating set is also a minimum edge dominating set, because for every graph $H$, the independent edge domination number $\gamma_{i}^{\prime}(H)$ is equal to the edge domination number $\gamma^{\prime}(H)$ :

Proof. Create a graph $G$ whose vertices correspond to the edges in $H$, where an edge between two vertices in $G$ if and only if those vertices correspond to incident edges in $H$. Then $H$ is line graph of $H$. Then an independent edge dominating set in $H$ corresponds to an independent vertex dominating set in $G$, so $\gamma_{i}^{\prime}(H)=\gamma_{i}(G)$. One property of line graphs is that they are claw-free, and Allen and Laskar proved that $\gamma_{i}$, the independent vertex domination number, is equal to $\gamma$, the vertex domination number, for all claw-free graphs [1]. Therefore, $\gamma_{i}(G)=\gamma(G)$. A minimum vertex dominating set in $G$ has size equal to $\gamma(G)$, and such a set corresponds to a minimum edge dominating set in $H$, so $\gamma(G)=\gamma^{\prime}(H)$. Therefore, $\gamma_{i}^{\prime}(H)=\gamma^{\prime}(H)$.

Then, as a Corollary to Theorem $8, \alpha^{T}(G)=n(G)-\gamma^{\prime}(G)$ for every graph $G$. We can use this information to find the total independence number of a graph if its edge domination number is known. For example, Mojdeh and Sadeghi proved that $\gamma^{\prime}\left(P_{3} \times P_{k}\right)=k$ in [20], and the number of vertices in this graph is $3 k$, so $\alpha^{T}\left(P_{3} \times P_{k}\right)=3 k-k=2 k$.

This also speaks to the difficulty of finding maximum total independent sets in graphs from certain families. We have seen that the problem of finding a maximum total independent set is equivalent to the problem of finding a minimum independent edge dominating set, because we can derive one from the other. Therefore, if it is easy to find a maximum total independent set, then it must be equally easy to find a minimum independent edge dominating set, which is also a minimum edge dominating set. Conversely, if it is difficult to find a minimum edge dominating set, then it must be just as hard to find a maximum total independent 
set.

For example, Yannakakis and Gavril showed in [28] that the edge dominating set problem is NP-complete for the general families of planar and bipartite graphs with $\Delta=3$. (This implies it is at least as hard for those with $\Delta>3$, and for non-planar, non-bipartite graphs.) Therefore, the maximum total independent set problem for those families must also be NP-complete.

On the other hand, those authors provided in the same paper a proof that the edge dominating set problem can be solved in linear time for trees. (This, they said, was an improvement over an earlier proof by Mitchell and Hedetniemi in [18].) If a minimum edge dominating set can be converted into a minimum independent edge dominating set in linear time, we can quickly convert it into a minimum total independent set, so the total independent set problem for trees would be solvable in a time frame of the same order.

\subsection{Total Independence Number of Hypercubes}

Theorem 9. For each integer $n \geq 3, \alpha^{T}\left(Q_{n}\right) \geq\left(\frac{5}{8}\right) 2^{n}$.

Proof. If $n=3$, this is the 3-cube $Q_{3}$, and each vertex corresponds to a coordinate in $\mathbb{Z}_{2}^{3}$, which will be its label. Let $T$ be a total independent set in $Q_{3}$ containing the vertices $(0,0,0)$ and $(1,1,1)$, as well as the edge from $(1,0,0)$ to $(1,1,0)$, the edge from $(0,0,1)$ to $(1,0,1)$, and the edge from $(0,1,0)$ to $(0,1,1)$. (These choices are illustrated on the left side of Figure 2.3.) Then $T$ has cardinality 5 , so $\alpha^{T}\left(Q_{k}\right) \geq 5$. As $\left(\frac{5}{8}\right) 2^{3}=5$, this satisfies the result of the theorem for $n=3$. 

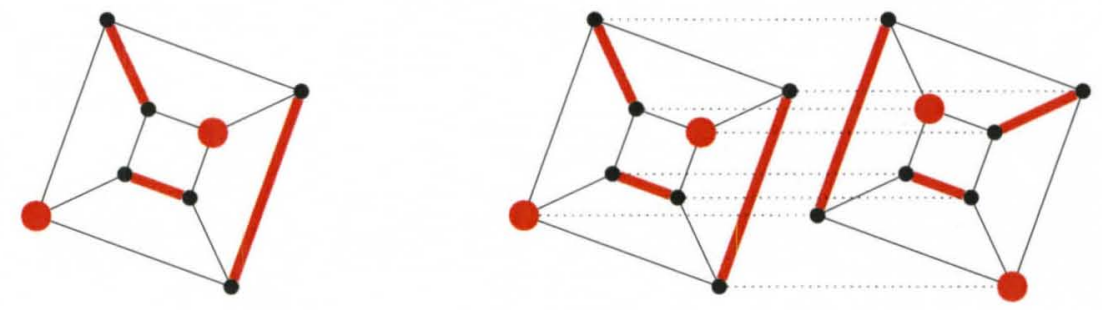

FIGURE 2.3: On the left, the 3-cube $Q_{3}$. On the right, the 4-cube $Q_{4}$. In each, a maximum total independent set is labeled in red.

If $n>3$, the theorem is proved by induction on $n$. Suppose the theorem is true for $n=k$. Give each vertex in $Q_{k+1}$ a label corresponding to its binary coordinate in $k+1$ dimensions. Observe that $Q_{k+1}$ contains two disjoint subgraphs isomorphic to $Q_{k}$ - one called $A$, induced by all vertices with a 0 in the $(k+1)^{\text {th }}$ position, and another called $B$, induced by all vertices with a 1 in the $(k+1)^{\text {th }}$ position. As $A$ is isomorphic to $Q_{k}$, the induction hypothesis states that $A$ contains a total independent set of size at least $\left(\frac{5}{8}\right) 2^{k}$. Choose a set $T$ of this size from the elements of $A$ in $Q_{k+1}$.

Now define the automorphism $r$ on the vertices of $Q_{k+1}$ : For each vertex $v \in Q_{k+1}, r(v)$ is the vertex in $Q_{k+1}$ whose coordinate is the same in every position except 1 and $k+1$, where it is different. This is clearly a one-to-one mapping. Let the set $R$ include $r(v)$ for every vertex $v \in T$. Every vertex $v \in T$ is in $A$, so the value of the position of $r(v)$ in $(k+1)^{t h}$ dimension must be 1 , therefore every vertex in $R$ is in $Q_{k+1} \backslash A$. Two vertices $u, v \in R$ cannot be adjacent, because if they are, then they must differ in only one dimension, and that imples vertices $r^{-1}(u)$ and $r^{-1}(v)$ differ in only one dimension (the same dimension), which is impossible. Therefore, $R$ is an independent set.

For every edge $u v \in T$, add the edge $r(u) r(v)$ to $R$. This adds a unique edge to $R$ for every edge in $T$, so now $R$ and $T$ include an equal number of vertices and include an equal number of edges. No two edges in $T$ share an endvertex, 
therefore no two edges in $R$ can share an endvertex, so the edges in $R$ constitute a matching. Also, no edge in $R$ can have an endvertex $v \in R$, because that would imply $r^{-1}(v)$ is in $T$ and also an endvertex of an edge in $T$, which is impossible. Therefore, $R$ is a total independent set.

A vertex $u \in R$ cannot be adjacent to a vertex $v \in T$ because that would imply $r^{-1}(u) \in T$ differs from $v$ in only the 1st dimension, so those vertices are adjacent, which is impossible. Therefore, the vertices in $T$ and $R$ together consitute an independent set. Every edge in $T$ has its endvertices in $A$, and every edge in $R$ has its endvertices in $Q_{k+1} \backslash A$, so the edges in $T$ and $R$ together constitute a matching. Finally, no endvertex of an edge in $T$ can be in $R$, and no endvertex of an edge in $R$ can be in $T$, so $T \cup R$ must be a total independent set in $Q_{k+1}$. (See Figure 2.3 for an illustration of this set in $Q_{4}$.) It has size equal to $2|T|=2\left(\frac{5}{8}\right) 2^{k}=\left(\frac{5}{8}\right) 2^{k+1}$, which makes this a lower bound for $\alpha^{T}\left(Q_{k+1}\right)$, which implies that the theorem is true for $n=k+1$.

Theorem 9 is not entirely satisfying because it only provides a lower bound for $\alpha^{T}\left(Q_{n}\right)$. By considering work that has been done in the field of maximal matchings, we can find an upper bound for it, but only for some values of $n$. In the 1970s, Forcade looked at the minimum maximal matchings of hypercubes and came up with several interesting results [6]. Using $m(G)$ to denote the size of a minimum maximal matching of a graph $G$, he found:

$$
m\left(Q_{n}\right) \geq \frac{n 2^{n}}{3 n-1} .
$$

The size $m$ of the minimum maximal matching is equal to the independent edge domination number $\gamma_{i}^{\prime}$, and the number of vertices in the $n$-cube is $2^{n}$, so we can use Forcade's result and Theorem 8 to produce an upper bound on the size of a total independent set in $Q_{n}$. 


\begin{tabular}{c|c|c|c|c}
$\begin{array}{c}\text { Dimension } \\
n\end{array}$ & $\begin{array}{c}\text { Forcade's } \\
\text { result }\end{array}$ & $\begin{array}{c}\text { Number of } \\
\text { Vertices }\end{array}$ & $\begin{array}{c}\text { Result of } \\
\text { Theorem 8 }\end{array}$ & $\begin{array}{c}\text { Result of } \\
\text { Theorem 9 }\end{array}$ \\
\hline 3 & $\gamma_{i}^{\prime} \geq 3$ & 8 & $\alpha^{T} \leq 5$ & $\alpha^{T} \geq 5$ \\
4 & $\gamma_{i}^{\prime} \geq 6$ & 16 & $\alpha^{T} \leq 10$ & $\alpha^{T} \geq 10$ \\
5 & $\gamma_{i}^{\prime} \geq 12$ & 32 & $\alpha^{T} \leq 20$ & $\alpha^{T} \geq 20$ \\
6 & $\gamma_{i}^{\prime} \geq 23$ & 64 & $\alpha^{T} \leq 41$ & $\alpha^{T} \geq 40$ \\
7 & $\gamma_{i}^{\prime} \geq 45$ & 128 & $\alpha^{T} \leq 83$ & $\alpha^{T} \geq 80$
\end{tabular}

TABLE 2.1: A comparison of some results for the hypercubes $Q_{3}$ through $Q_{7}$.

Table 2.1 establishes the total independence number of the hypercube for dimensions 3, 4, and 5. For higher dimensions, this is not as clear, because the upper bound for $\alpha^{T}$ is greater than the lower bound found in Theorem 9. This opens up the possibility that, for high enough values of $n$, a total independent set in $Q_{n}$ can exist with size greater than $\left(\frac{5}{8}\right) 2^{n}$.

Observe that the proof of Theorem 9 involves setting up a total independent set $T$ in $Q_{n}$ of size $\left(\frac{5}{8}\right) 2^{n}$. Every vertex of $Q_{n}$ is either in $T$ or is an endvertex of an edge in $T$, so the edges in $T$ constitute a maximal matching $M$. These edges make up 3 out of every 5 elements in $T$, so $|M|=\left(\frac{3}{8}\right) 2^{n}$. If $M$ is smallest among all maximal matchings in $Q_{n}$, then $\gamma_{i}^{\prime}=|M|$, and it follows from Theorem 8 that $\alpha^{T}\left(Q_{n}\right)=2^{n}-|M|=\left(\frac{5}{8}\right) 2^{n}$, and the bound expressed in Theorem 9 is sharp. But if a maximal matching smaller than $M$ can be found, then $\gamma_{i}^{\prime}\left(Q_{n}\right)<|M|$, and $\alpha^{T}\left(Q_{n}\right)>2^{n}-|M|=\left(\frac{5}{8}\right) 2^{n}$, so the total independence number would exceed that lower bound.

In the case of dimension $n=6$, Havel and Krivanel proved in [8] that a maximal matching in $Q_{n}$ must have size at least 24, which means (1) Forcade's lower bound on $m\left(Q_{n}\right)$ is not sharp for some values of $n>5$, and (2) with the result of Theorems 8 and 9 , this implies $\alpha^{T}\left(Q_{6}\right)=40$. 
In [6], Forcade found another way to construct a maximal matching in $Q_{n}$. For small values of $n$, his constructions are larger than the maximal matching $M$ in $Q_{n}$ found by Theorem 9, but Forcade shows that the size of his maximal matching approaches $\left(\frac{1}{3}\right) 2^{n}$ as $n$ approaches infinity. Therefore, some $n$ must exist for which $\gamma_{i}^{\prime}\left(Q_{n}\right)<\left(\frac{3}{8}\right) 2^{n}$. In particular, when $n=48$, his matching uses about 667 billion edges less than $M$ does (although, for $n=47$, his matching uses about 69 billion more). We can see, then, that Theorem 9 is definitely not sharp for $n \geq 48$. It may not even be sharp for dimensions as low as $n=7$, because $m\left(Q_{7}\right)$ is still unknown. 


\section{CHAPTER 3 TOTAL CHROMATIC SUM}

\subsection{History of the Chromatic Sum}

Instead of trying to keep a small number of color classes, suppose our goal is to make specific color classes as large as possible, or to collect as many large color classes as possible. This is a vague demand without a well-defined goal or function to optimize, so let us define this demand in a very specific way: Define the weight of an element to be a function of its color; there are many options for this function, but the simplest non-trivial choice is a linear one: Color 1 has weight 1 , color 2 has weight 2, color $n$ has weight $n$, etc. Then define the weight of a coloring to be the sum of the weights of all its colored elements. Given a graph, we want to find a coloring that minimizes this weight.

This weighting system can be applied to a vertex, edge, or total coloring. In this section, some of the results for vertex and edge colorings will be discussed.
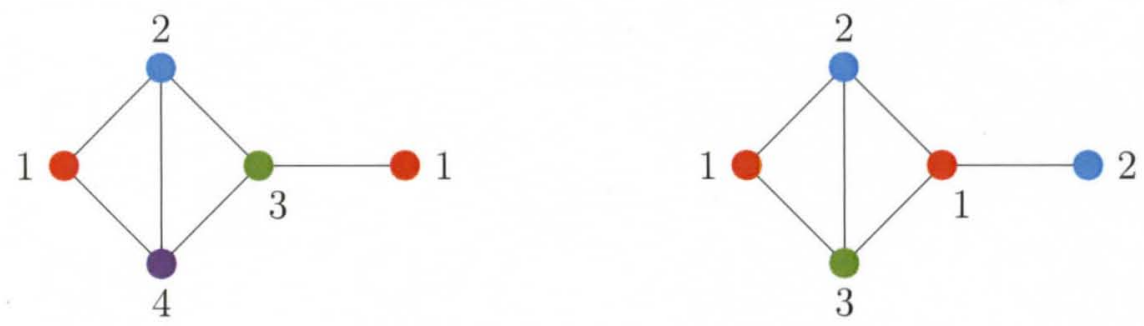

FIGURE 3.1: A graph shown twice, with two different colorings. The coloring on the left has weight 11 , and the coloring on the right has weight 9 . 
Suppose we want to color the vertices of the graph in Figure 3.1 in a way that minimizes the weight of the coloring. A naïve approach would be to assign color 1 to as many vertices as possible (a maximum independent set, perhaps), then give color 2 to as many uncolored elements as possible, and so on, until all elements are colored. A simple greedy algorithm designed to make these color class selections one at a time will not necessarily produce a coloring of minimum weight, because the choice we make for each color class - not just the size - affects the potential choices for the higher color classes. For example, in Figure 3.1, the graph shown has independence number 2 , but we can choose the first color class in two ways. If the first color class is chosen as shown on the left, then the remaining vertices form a triangle, so the coloring must use three additional colors and have weight at least $1+1+2+3+4=11$. But if the first color class is chosen as shown on the right, then a coloring can be made with three colors, which has weight $1+1+2+2+3=9$.

The smallest possible weight that a coloring of a graph $G$ can have is called its chromatic sum, and this is denoted $\Sigma(G)$. A coloring is optimal if it has weight equal to $\Sigma(G)$. This idea was introduced by Kubicka in 1989 [15], and since then much more work has been done with calculating the total chromatic sums of graphs, generating algorithms to find total chromatic sums and optimal colorings, and calculating the complexity of finding total chromatic sums of graphs in certain families. In [5], Erdös, Kubicka, and Schwenk examined cases in which the minimum number of colors necessary to make an optimal coloring of $G$ was larger than $\chi(G)$. This graph parameter, the minimum number of colors necessary for an optimal coloring, was called the strength of $G$, and denoted by $\sigma(G)$ [12]. (Note that this is different from the parameter of the same name that applies to directed graphs). In [5], it is shown that even trees can have arbitrarily high strength, even though their chromatic number is 2 . 

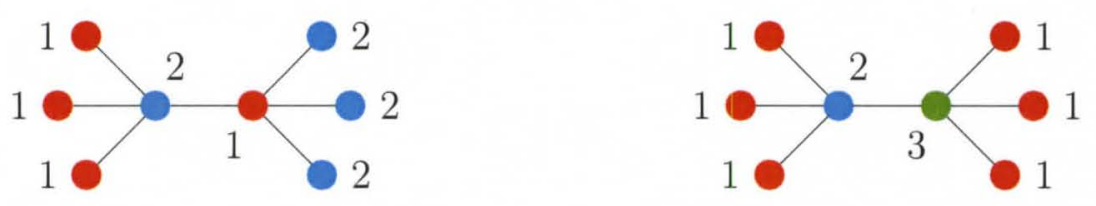

FIGURE 3.2: A graph shown twice, with different colorings. The coloring on the left has weight 12 , and uses only $\chi=2$ colors. The coloring on the right has weight $\Sigma=11$, but uses 3 colors.

An example of a tree with high strength is given in Figure 3.2. This tree has chromatic number 2 , but if it is colored with that many colors, as shown on the left, then the coloring has weight 12 . If a third color is allowed, a coloring can be made with weight 11, so the chromatic sum of the graph is at most 11. Since this weight is impossible to obtain with two colors, the strength of the graph must be at least 3 , but that is greater than the chromatic number.

Naturally, mathematicians have applied all of these color-sum concepts to edge coloring as well. Of particular interest is the edge chromatic sum of a graph, its edge strength $\sigma^{\prime}$, and the question of whether or not $\chi^{\prime}=\sigma^{\prime}$. In 1997, Mitchem, Morriss, and Schmeichel showed in [19] that every graph has a proper edge coloring with minimum sum that uses only $\Delta$ or $\Delta+1$ colors. This implies that the only way for a graph to have $\chi^{\prime}<\sigma^{\prime}$ is to have both $\chi^{\prime}=\Delta$ and $\sigma^{\prime}=\Delta+1$. In [7], Giaro gave an example of a graph with this property, shown in Figure 3.3. On the left, $G$ is edge colored with $\Delta(G)=\chi^{\prime}(G)=5$ colors. If only five colors are used, the weight of the coloring must be at least 45 . If we introduce a sixth color, and change the colors of the edges whose color labels are circled, we obtain a coloring with weight 43 , so the edge chromatic sum of $G$ is at most 43 . Since this weight is impossible to obtain with five colors, the edge strength of the graph must be at least 6 . 

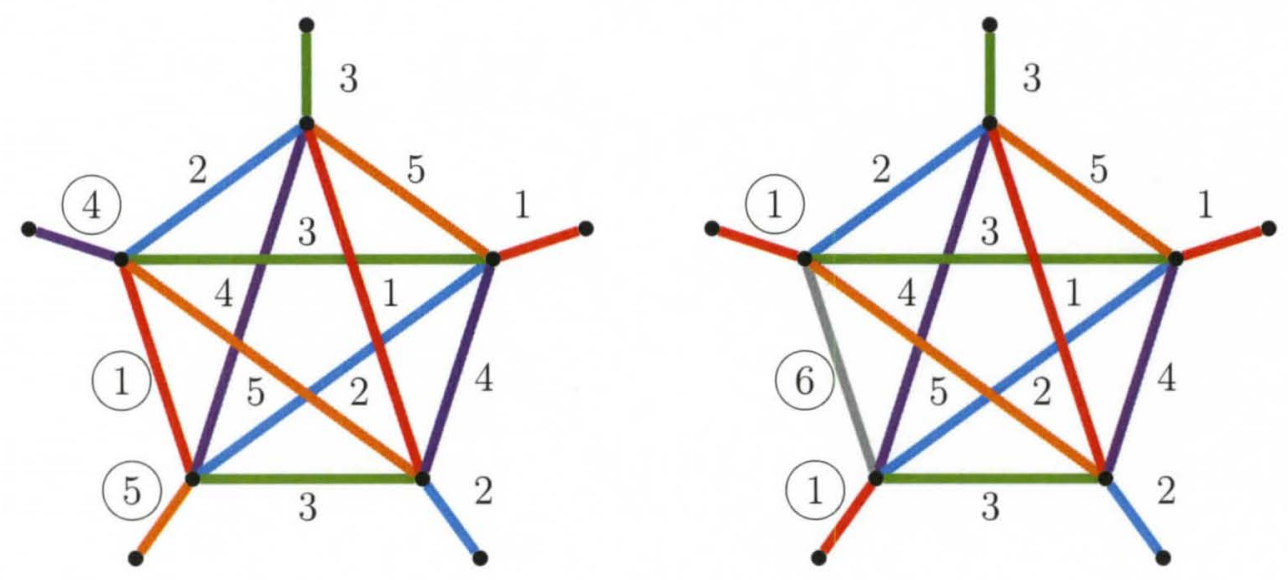

FIGURE 3.3: Giaro's example of a graph with $\Delta=5, \chi^{\prime}=5$, and $\sigma^{\prime} \geq 6$.

\subsection{Total Colorings with Optimal Sum}

Now this concept will be applied to total coloring. For every graph $G$, define the function sum : $\Phi(G) \rightarrow \mathbb{N}$ to return the weight of a coloring $\phi$ of $G$ :

$$
\operatorname{sum}(\phi)=\sum_{x \in V(G) \cup E(G)} \phi(x) .
$$

The total chromatic sum $\Sigma^{T}(G)$ of a graph $G$ is defined as the smallest value of $\operatorname{sum}(\phi)$ among all colorings $\phi \in \Phi(G)$ :

$$
\Sigma^{T}(G)=\min _{\phi \in \Phi(G)} \operatorname{sum}(\phi) .
$$

As in other coloring methods, we will call a total coloring $\phi$ of $G$ optimal if $\operatorname{sum}(\phi)=\Sigma^{T}(G)$. For each total coloring $\phi$, define $\sigma(\phi)$ to be the number of colors used in $\phi$; then the total strength of $G$ can be defined as the smallest value of $\sigma(\phi)$ among all optimal total colorings $\phi$ of $G$, and this is denoted $\sigma^{T}(G)$ :

$$
\sigma^{T}(G)=\min \left\{\sigma(\phi): \phi \in \Phi(G) \text { and } \operatorname{sum}(\phi)=\Sigma^{T}(G)\right\} .
$$

The primary purpose of this chapter will be to calculate $\Sigma^{T}(G)$ and $\sigma^{T}(G)$ for some simple families of graphs. But first, a few general observations should be 
made about the color classes that must appear in an optimal total coloring $\phi$ of $G$. First, $\phi$ cannot assign color 2 to more elements than color $1: \phi_{1} \geq \phi_{2}$. Otherwise, we could permute the colors 1 and 2 in $\phi$ to produce a coloring with an even smaller sum. In general, if $i<j$, then $\phi$ cannot assign color $j$ to more elements than color $i$, so $i<j$ implies $\phi_{i} \geq \phi_{j}$. Second, every color class in $\phi$ must constitute a total independent set, therefore its size is bounded from above by $\alpha^{T}(G)$. In other words, $\phi_{i} \leq \alpha^{T}$ for every color $i$. We can combine these observations into the following inequalities:

$$
\alpha^{T}(G) \geq \phi_{1} \geq \phi_{2} \geq \ldots \geq \phi_{\chi^{T}(G)} \geq \ldots \geq \phi_{\sigma} .
$$

These inequalites are necessary, but not sufficient, for a coloring to be optimal. In the next section, some conditions will be developed to guarantee that a coloring is optimal.

\subsection{Sufficiency Conditions for Optimality}

For each graph $G$, and for each $(k, m) \in \mathbb{N} \times \mathbb{N}$, we will say that " $G$ has property $R_{k, m}$ " if $\phi_{k} \leq m$ in every total coloring $\phi$ of $G$.

Theorem 10. Let $G$ be a graph with $\alpha^{T}=\alpha^{T}(G)$ and $\chi^{T}=\chi^{T}(G)$. If $G$ has the property $R_{p}$ for every $p \in\left\{\left(k_{1}, m_{1}\right),\left(k_{2}, m_{2}\right), \ldots,\left(k_{z}, m_{z}\right)\right\}$ (with $k_{i}<k_{i+1}$ and $m_{i} \geq m_{i+1}$ for every $i<z$ ), and if $G$ has a coloring $\phi$ such that $\phi_{n}=\alpha^{T}$ for all $n<k_{1}, \phi_{n}=m_{i}$ whenever $k_{i} \leq n<k_{i+1}$ for an integer $i \in[1, z-1]$, and $\phi_{n}=m_{z}$ whenever $k_{z} \leq n<\sigma(\phi)$, then $\phi$ is optimal.

Proof. Suppose $G$ has the stated properties and $\phi$ satisfies the given conditions. By the definition of the total chromatic parameter, $\sigma(\phi) \geq \chi^{T}$ must be true. Now it will be shown that $\sigma(\phi)=\chi^{T}$ : Suppose $\sigma(\phi)>\chi^{T}$. Then a total coloring $\psi$ 
exists such that $\sigma(\psi)=\chi^{T}<\sigma(\phi)$. In each coloring, the sum of all color classes is equal to $|V \cup E|$ :

$$
\begin{gathered}
\sum_{n=1}^{\sigma(\phi)} \phi_{n}=|V+E|=\sum_{n=1}^{\sigma(\psi)} \psi_{n} \\
\sum_{n=1}^{\sigma(\psi)} \phi_{n}+\sum_{n=\sigma(\psi)+1}^{\sigma(\phi)} \phi_{n}=\sum_{n=1}^{\sigma(\psi)} \psi_{n} \\
\sum_{n=1}^{\sigma(\psi)} \phi_{n}<\sum_{n=1}^{\sigma(\psi)} \psi_{n} .
\end{gathered}
$$

For this to be true, there must be some color $c \leq \sigma(\psi)$ such that $\phi_{c}<\psi_{c}$. Color $c$ cannot be less than $k_{1}$ because that would imply $\psi_{c}>\phi_{c}=\alpha^{T}$, which is impossible. Color $c$ cannot be in an interval $p=\left[k_{i}, k_{i+1}\right)$ for which $G$ has the property $R_{p}$, because that would imply $\psi_{c}>\phi_{c}=m_{i}$, and $R_{p}$ makes this impossible. As $c \leq \sigma(\psi)<\sigma(\phi)$, color $c$ can only be in the interval $\left[k_{z}, \sigma(\phi)\right)$, but that implies $\psi_{c}>\phi_{c}=m_{z}$, which is impossible. Thus $\phi_{c}<\psi_{c}$ is impossible for every value of $c$, no such $\psi$ can exist. The contradiction implies $\sigma(\phi)=\chi^{T}$.

Now suppose $\psi$ is a coloring of $G$ with $\operatorname{sum}(\psi)<\operatorname{sum}(\phi)$. Given this freedom, we can and will choose $\psi$ so that it is optimal. The sums are different, so $\phi_{d} \neq \psi_{d}$ for some integer $d$; let $d$ be the least integer for which $\phi_{d} \neq \psi_{d}$. If $d<\sigma(\phi)$, then there is an integer $i \in[1, z]$ such that $\phi_{d}=m_{i}$ and $d \geq k_{i}$, and $R_{\left(k_{i}, m_{i}\right)}$ states that $\psi_{k_{i}} \leq m_{i}$, so $\psi_{d} \leq \psi_{k_{i}} \leq m_{i}=\phi_{i}$; therefore, $\psi_{d}<\phi_{d}$. The same is true if $d \geq \sigma(\phi):$

$$
\psi_{d}=|V \cup E|-\sum_{n<d} \psi_{n}-\sum_{n>d} \psi_{n} \leq|V \cup E|-\sum_{n<d} \psi_{n}=\sum_{n=1}^{d} \phi_{n}-\sum_{n<d} \phi_{n}=\phi_{d} .
$$

So $\psi_{d}<\phi_{d}$ in every case. Then: 


$$
\begin{aligned}
\operatorname{sum}(\psi)-\operatorname{sum}(\phi) & =\sum_{n} n \psi_{n}-\sum_{n} n \phi_{n} \\
& =\sum_{n} n\left(\psi_{n}-\phi_{n}\right) \\
& =\sum_{n<d} n\left(\psi_{n}-\phi_{n}\right)+d\left(\psi_{d}-\phi_{d}\right)+\sum_{n>d} n\left(\psi_{n}-\phi_{n}\right) \\
& =0+d\left(\psi_{d}-\phi_{d}\right)+\sum_{n>d} n\left(\psi_{n}-\phi_{n}\right) \\
& \geq d\left(\psi_{d}-\phi_{d}\right)+(d+1) \sum_{n>d}\left(\psi_{n}-\phi_{n}\right) \\
& \geq d\left(\psi_{d}-\phi_{d}\right)+(d+1)\left(\sum_{n}\left(\psi_{n}-\phi_{n}\right)-\left(\psi_{d}-\phi_{d}\right)-\sum_{n<d}\left(\psi_{n}-\phi_{n}\right)\right) \\
& \geq d\left(\psi_{d}-\phi_{d}\right)+(d+1)\left(0-\left(\psi_{d}-\phi_{d}\right)-0\right) \\
& \geq \phi_{d}-\psi_{d}>0 \\
\operatorname{sum}(\psi) & >\operatorname{sum}(\phi) .
\end{aligned}
$$

This contradicts the definition of $\psi$ as a coloring with sum less than $\phi$, so no such coloring exists; therefore, $\phi$ is optimal.

Theorem 10 can be used to prove the optimality of some very simple total colorings for paths, cycles, and complete graphs, and this will be shown in the next section. However, for these graphs, this theorem is much too general, because most of these graphs have no restrictions of the form $R_{(k, m)}$, and some only have one. The following corollaries are provided for simple cases like these.

First Corollary to Theorem 10. If a graph $G$ has a coloring $\phi$ such that $\phi_{n}=\alpha^{T}(G)$ for every color $n<\sigma(\phi)$, then $\phi$ is optimal.

Proof. Putting the restriction $\phi_{1} \leq \alpha^{T}$ into the language of the restriction set of Theorem 10, $G$ has the property $R_{p}$ for every $p \in\left\{\left(k_{1}, m_{1}\right)\right\}$ where $k_{1}=1$ and $m_{1}=\alpha^{T}$. In this set of restrictions, $z=1$, so the given coloring $\phi$ has the properties 
$\phi_{n}=\alpha^{T}$ for all $n<k_{1}$ (an empty set), $\phi_{n}=m_{i}$ whenever $k_{i} \leq n<k_{i+1}$ for an integer $i \in[1, z-1]$ (an empty set), and $\phi_{n}=m_{z}$ whenever $k_{z} \leq n<\sigma(\phi)$ - which is precisely the information we have about $\phi$. Then, by Theorem $10, \phi$ is optimal.

Second Corollary to Theorem 10 . Let $G$ be a graph. If some $k, m \in \mathbb{N}$ exist such that every coloring $\psi$ of $G$ has the property $\psi_{k} \leq m$, and if $G$ has a coloring $\phi$ such that $\phi_{n}=\alpha^{T}(G)$ for all $n<k$ and $\phi_{n}=m$ whenever $k \leq n<\sigma(\phi)$, then $\phi$ is optimal.

Proof. Putting the restrictions $\phi_{1} \leq \alpha^{T}$ and $\phi_{k} \leq m$ into the language of the restriction set of Theorem $10, G$ has the property $R_{p}$ for every $p \in\left\{\left(1, \alpha^{T}\right),(k, m)\right\}$. In this set of restrictions, $z=2$, so the given coloring $\phi$ has the properties $\phi_{n}=\alpha^{T}$ for all $n<k_{1}$ (an empty set), $\phi_{n}=m_{i}=\alpha^{T}$ whenever $k_{i} \leq n<k_{i+1}$ for an integer $i \in[1, z-1]$ (corresponding to all $n<k$ ), and $\phi_{n}=m_{z}=m$ whenever $k_{z} \leq n<\sigma(\phi)$ (corresponding to all integers $n$ that are at least $k$ but less than $\sigma(\phi)$ ). This is precisely the information we have about $\phi$, so by Theorem $10, \phi$ is optimal.

We can already quantify the color classes of a coloring $\phi$, but doing so by stating the values of each quantity $\phi_{1}, \phi_{2}$, et cetera, can be cumbersome. These quantities make up a sequence, so it should not be alien to write the color classes of $\phi$ in a vector form $\left\langle\phi_{1}, \phi_{2}, \phi_{3}, \ldots, \phi_{\sigma}\right\rangle$. This will be called the color class sequence of $\phi$. With this notation, we could restate the First Corollary to Theorem 10 to say " $\phi$ is an optimal coloring of $G$ if it has the color class sequence $\left\langle\alpha^{T}(G), \ldots, \alpha^{T}(G), p\right\rangle$. This notation will be used in the proof of the next theorem. 
Theorem 11. Let $G$ be a graph with total chromatic number $\chi^{T}$ and the property that, for some integer $m \leq \alpha^{T}(G)$,

$$
\sum_{n>c} \psi_{n} \geq m\left(\chi^{T}-c\right)
$$

for every coloring $\psi$ of $G$ and for every color $c \leq \chi^{T}$. If an integer $k>1$ and $a$ coloring $\phi$ exists such that $\phi_{n}=\alpha^{T}(G)$ for all colors $n$ from 1 to $k-1, \phi_{n}=m$ for all colors $n$ from $k+1$ to $\chi^{T}$, and $\phi_{n}=0$ for all $n>\chi^{T}$, then $\phi$ is optimal.

Proof. Suppose $G$ is a graph satisfying this condition and $\phi$ is a coloring of $G$ with the stated properties. Set up the sequence $\left\{v_{n}\right\}$ such that $v_{c}=\phi_{c}$ for every color c. Let $\psi$ be a coloring that is different from $\phi$.

Define the operation $\operatorname{push}(v, c)$ to change the sequence $\left\{v_{n}\right\}$ in the following way: $\operatorname{push}(v, c)$ reduces $v_{c}$ by one and increases $v_{c+1}$ by one. This operation has several important properties: First, it cannot change the sum $\sum_{n} v_{n}=|V \cup E|$ - Second, the sum $\sum_{n} n v_{n}$ begins equal to $\operatorname{sum}(\phi)$, and each instance of push increases that sum by one.

Using the following algorithm, we can change $\left\{v_{n}\right\}$ to match the color class sequence of $\psi$ with a repeated application of $\operatorname{push}(v, c)$ :

1. Set the variable $c$ to 1 .

2. If $v_{c}>\psi_{c}$, repeat $\operatorname{push}(v, c)$ until $v_{c}=\psi_{c}$.

3. Increase $c$ by 1 ; if $v_{c}>0$, return to step 2 .

Observe that, whenever step 2 begins, $v_{c}$ is greater than or equal to $\psi_{c}$ for every value of $c$. When $c<k$, this is because $v_{c}$ begins at $\alpha^{T}$ and the algorithm can't decrease it before this step (and $\psi_{c} \leq \alpha^{T}$ ). If $c \geq k$, it is because at this point in the algorithm, $v_{n}=\psi_{n}$ for every $n<c$ and $v_{n}=\phi_{n}$ for every $n>c$, so: 


$$
\begin{aligned}
v_{c} & =|V \cup E|-\sum_{n<c} v_{n}-\sum_{n>c} v_{n} \\
& =|V \cup E|-\sum_{n<c} \psi_{n}-\sum_{n>c} \phi_{n} \\
& =\sum_{n \geq c} \psi_{n}-\sum_{n=c+1}^{\chi^{T}} m \\
& =\psi_{c}+\sum_{n>c} \psi_{n}-\sum_{n=c+1}^{\chi^{T}} m \\
& \geq \psi_{c}+m\left(\chi^{T}-c\right)-\left(\chi^{T}-c\right) m=\psi_{c} .
\end{aligned}
$$

The algorithm will repeat step 2 until $v_{c}=\psi_{c}$, at which point it moves to a higher color, and $v_{c}$ will not be altered again. It is a consequence of 3.1 that $\psi$ cannot use more than $|V \cup E|$ colors, so $v_{c}$ will become 0 by the time $c=|V \cup E|$, and step 2 can only repeat itself a finite number of times, so $c$ will continue to increase and the algorithm will eventually terminate. At that time, we will have $v_{c}=\psi_{c}$ for every color $c$.

Note that, because $\phi \neq \psi$, the push operation must have been used at least once. Then $\operatorname{sum}(\psi)=\sum_{n} n \psi_{n}=\sum_{n} n v_{n}=\operatorname{sum}(\phi)+($ the number of times push was used), so $\operatorname{sum}(\psi)>\operatorname{sum}(\phi)$. Incidentally, this proves that an optimal coloring of $G$ must have the same color class sequence as $\phi$.

The lemmas of this section could also be used in coloring contexts other than total coloring, such as vertex or edge coloring, and the same results can be obtained if every mention of total coloring, total independence number, and total chromatic number is replaced with the analogous term appropriate to the context. 


\subsection{Total Chromatic Sum of Some Common Graphs}

Theorem 12. For each integer $n>1$, it is true that $\sigma^{T}\left(P_{n}\right)=3$ and

$$
\Sigma^{T}\left(P_{n}\right)= \begin{cases}4 n-2 & \text { if } n \equiv 2(\bmod 3), \\ 4 n-3 & \text { otherwise. }\end{cases}
$$

Proof. Let $n$ be an integer greater than 1. It is easy to see that $\chi^{T}\left(P_{n}\right)=3$, and we already have $\alpha^{T}=\alpha^{T}\left(P_{n}\right)=\left\lceil\frac{2 n-1}{3}\right\rceil$ (Theorem 2). Label the vertices of $P_{n}$ as $v_{1}, v_{2}$, etc., to $v_{n}$. Construct the total coloring $\phi$ so that $v_{1}$ has color $1, v_{1} v_{2}$ has color $2, v_{2}$ has color 3 , and for every integer $i \in[2, n), v_{i} v_{i+1}$ gets the color in $\{1,2,3\} \backslash\left\{v_{i-1} v_{i}, v_{i}\right\}$ and $v_{i+1}$ gets the color in $\{1,2,3\} \backslash\left\{v_{i}, v_{i} v_{i+1}\right\}$.

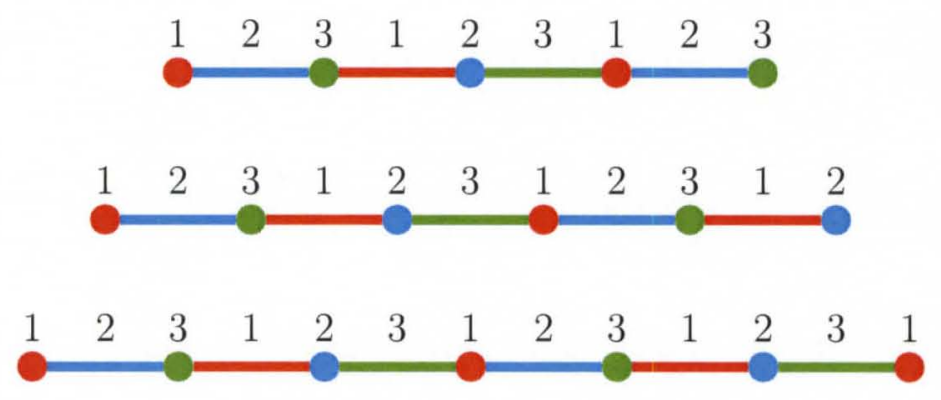

FIGURE 3.4: A demonstration of $\phi$ on paths of three modularities.

Figure 3.4 demonstrates the coloring $\psi$ on paths of various lengths. Color 1 is always used exactly $\left[\frac{2 n-1}{3}\right\rceil$ times, so $\phi_{1}=\alpha^{T}$ and $\sigma(\phi)=3$. However, color 2 is used $\left\lceil\frac{2 n-2}{3}\right\rceil$ times, and color 3 is used $\left\lceil\frac{2 n-3}{3}\right\rceil$ times, so $\phi_{1}$ and $\phi_{2}$ will vary with the modularity of $n$ :

Case $1: \quad n \equiv 0(\bmod 3)$; then $2 n \equiv 0(\bmod 3)$, so $\phi_{2}=\left\lceil\frac{2 n-2}{3}\right\rceil=\left\lceil\frac{2 n}{3}-\frac{2}{3}\right\rceil=\left\lceil\frac{2 n}{3}-\frac{1}{3}\right\rceil$ $=\left\lceil\frac{2 n-1}{3}\right\rceil=\alpha^{T}$, and $\phi_{3}=\left\lceil\frac{2 n-3}{3}\right\rceil=\frac{2 n}{3}-1=\left\lceil\frac{2 n}{3}-\frac{1}{3}\right\rceil-1=\left\lceil\frac{2 n-1}{3}\right\rceil-1=\alpha^{T}-1$. Then $\operatorname{sum}(\phi)=\alpha^{T}+2 \alpha^{T}+3\left(\alpha^{T}-1\right)=6\left(\frac{2 n}{3}\right)-3=4 n-3$. 
Case 2: $n \equiv 2(\bmod 3)$; then $2 n \equiv 1(\bmod 3)$, so $\phi_{2}=\left\lceil\frac{2 n-2}{3}\right\rceil=\left\lceil\frac{2 n-1}{3}\right\rceil=\alpha^{T}$, and $\phi_{3}=\left\lceil\frac{2 n-3}{3}\right\rceil=\left\lceil\frac{2 n-1}{3}\right\rceil=\alpha^{T}$. Then $\operatorname{sum}(\phi)=\alpha^{T}+2 \alpha^{T}+3 \alpha^{T}=6\left(\frac{2 n-1}{3}\right)=4 n-2$.

Case 3: $n \equiv 1(\bmod 3)$; then $2 n \equiv 2(\bmod 3)$, so $\phi_{2}=\left\lceil\frac{2 n-2}{3}\right\rceil=\left\lceil\frac{2 n-1}{3}\right\rceil-1=\alpha^{T}-1$. Then $\operatorname{sum}(\phi)=\alpha^{T}+2\left(\alpha^{T}-1\right)+3\left(\alpha^{T}-1\right)=6\left\lceil\frac{2 n-1}{3}\right\rceil-5=6\left(\frac{2 n-2}{3}+\left\lceil\frac{1}{3}\right\rceil\right)-5=$ $4 n-4+6(1)-5=4 n-3$. This path has only one total independent set of size $\alpha^{T}$, namely $\left\{v 1, v_{2} v_{3}, v_{4}, \ldots, v_{n-3}, v_{n-2} v_{n-1}, v_{n}\right\}$. This implies $\phi_{2}<\alpha^{T}$ in every coloring.

Given the color class sequences found, we can use the First Corollary to Theorem 10 to show that $\phi$ is optimal in cases 1 and 2 . In case $3, \phi$ is optimal by the Second Corollary, using the condition $\phi_{2} \leq \alpha^{T}-1$. Then $\phi$ is optimal in all cases of $n>1$. Three colors are both necessary and sufficient to obtain an optimal coloring in each case, so $\sigma^{T}\left(P_{n}\right)=3$.

Theorem 13. For each integer $n \geq 3$, it is true that $\sigma^{T}\left(C_{n}\right)=\chi^{T}\left(C_{n}\right)$ and

$$
\Sigma^{T}\left(C_{n}\right)= \begin{cases}4 n & \text { if } n \equiv 0(\bmod 3), \\ 4 n+4 & \text { if } n \equiv 1(\bmod 3), \\ 4 n+2 & \text { if } n \equiv 2(\bmod 3) .\end{cases}
$$

Proof. Let $C_{n}$ be the cycle on $n \geq 3$ vertices. We already have $\alpha^{T}=\alpha^{T}\left(C_{n}\right)=\lceil 2 n / 3\rceil$ (Theorem 3). Label the vertices of the cycle $v_{0}, v_{1}, v_{2}$, etc., so that $v_{n}=v_{0}$. Now continue with one of three cases, depending on the modularity of $n$ :
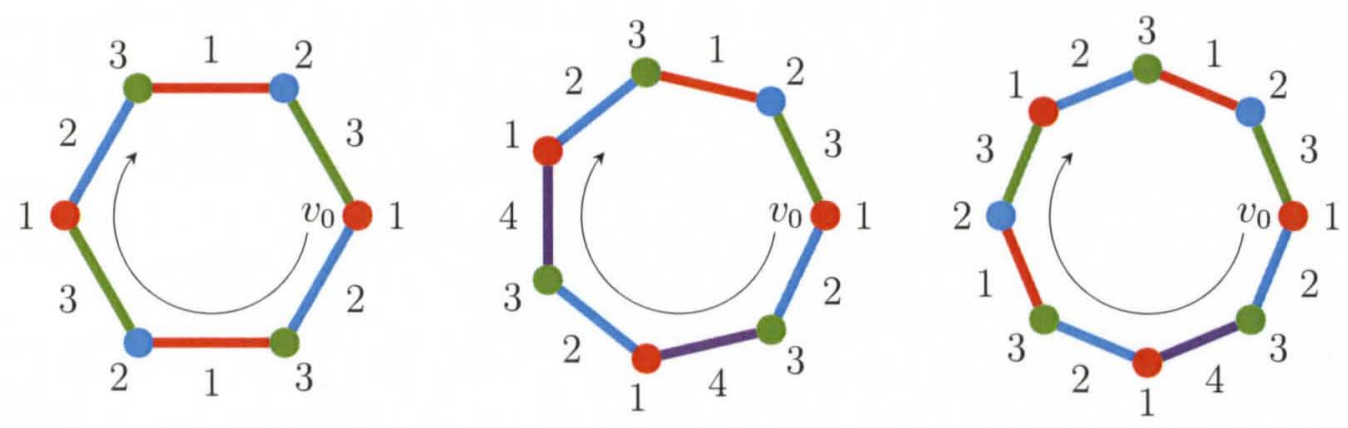

FIGURE 3.5: A demonstration of $\phi$, as described below, on cycles of three modularities. 
Case 1: $n \equiv 0(\bmod 3)$; Yap shows in [29] that this is the only case in which $\chi^{T}\left(C_{n}\right)=3$. Construct the total coloring $\phi$ so that $v_{0}$ has color $1, v_{0} v_{1}$ has color 2 , and for every integer $i \in[2, n), v_{i}$ gets the color in $\{1,2,3\} \backslash\left\{v_{i-1}, v_{i-1} v_{i}\right\}$ and $v_{i} v_{i+1}$ gets the color in $\{1,2,3\} \backslash\left\{v_{i-1} v_{i}, v_{i}\right\}$. Since the number of elements, $2 n$, is divisible by 3 , each of the 3 colors is assigned to exactly $\frac{2 n}{3}=\alpha^{T}$ elements. Then $\operatorname{sum}(\phi)=\alpha^{T}+2 \alpha^{T}+3 \alpha^{3}=6\left(\frac{2 n}{3}\right)=4 n$. This coloring is optimal by Theorem 9, and uses the minimum number of colors, so $\sigma^{T}\left(C_{n}\right)=\chi^{T}\left(C_{n}\right)=3$.

Case 2: $\quad n \equiv 1(\bmod 3)$; in this case, $\chi^{T}\left(C_{n}\right)=4$. Construct $\phi$ so that $\phi\left(\left\langle v_{0}, v_{0} v_{1}, \ldots, v_{3}, v_{3} v_{4}\right\rangle\right)=\langle 1,2,3,4,1,2,3,4\rangle$, and for every integer $i \in[4, n), v_{i}$ gets the color in $\{1,2,3\} \backslash\left\{v_{i-1}, v_{i-1} v_{i}\right\}$ and $v_{i} v_{i+1}$ gets the color in $\{1,2,3\} \backslash\left\{v_{i-1} v_{i}, v_{i}\right\}$. In this way, Color 1 is assigned to $v_{0}, v_{2}$, and $\frac{2 n-8}{3}$ other elements, so $\phi_{1}=$ $2+\frac{2 n-2}{3}-\frac{6}{3}=\frac{2 n-2}{3}=\lfloor 2 n / 3\rfloor=\alpha^{T}$. Similarly, $\phi_{2}=\phi_{3}=\alpha^{T}$, but color 4 is only assigned to two elements. Then $\operatorname{sum}(\phi)=\alpha^{T}+2 \alpha^{T}+3 \alpha^{T}+4(2)=6\left(\frac{2 n-2}{3}\right)+8=4 n+4$. This coloring is optimal by Theorem 9 , and uses the minimum number of colors, so $\sigma^{T}\left(C_{n}\right)=\chi^{T}\left(C_{n}\right)=4$.

Case 3: $\quad n \equiv 2(\bmod 3)$; in this case, $\chi^{T}\left(C_{n}\right)=4$. Construct $\phi$ so that $\phi\left(\left\langle v_{0}, v_{0} v_{1}, v_{1}, v_{1} v_{2}\right\rangle\right)=\langle 1,2,3,4\rangle$, and for every integer $i \in[2, n), v_{i}$ gets the color in $\{1,2,3\} \backslash\left\{v_{i-1}, v_{i-1} v_{i}\right\}$ and $v_{i} v_{i+1}$ gets the color in $\{1,2,3\} \backslash\left\{v_{i-1} v_{i}, v_{i}\right\}$. In this way, Color 1 is assigned to $v_{0}$ and $\frac{2 n-4}{3}$ other elements, so $\phi_{1}=1+\frac{2 n-1}{3}-\frac{3}{3}=$ $\frac{2 n-1}{3}=\lfloor 2 n / 3\rfloor=\alpha^{T}$. Similarly, $\phi_{2}=\phi_{3}=\alpha^{T}$, but color 4 is only assigned to one element. Then $\operatorname{sum}(\phi)=\alpha^{T}+2 \alpha^{T}+3 \alpha^{T}+4(1)=6\left(\frac{2 n-1}{3}\right)+4=4 n+2$. This coloring is optimal by Theorem 9, and uses the minimum number of colors, so $\sigma^{T}\left(C_{n}\right)=\chi^{T}\left(C_{n}\right)=4$.

Theorem 14. For each integer $n \geq 1$, it is true that $\sigma^{T}\left(K_{n}\right)=\chi^{T}\left(K_{n}\right)$, and

$$
\Sigma^{T}\left(K_{n}\right)= \begin{cases}\frac{n(n+1)^{2}}{4} & \text { if } n \text { is odd, } \\ \frac{n(n+1)(n+2)}{4} & \text { if } n \text { is even. }\end{cases}
$$


Proof. Theorem 4 tells us $\alpha^{T}\left(K_{n}\right)=\lceil n / 2\rceil$. In [29], Yap shows that $\chi^{T}\left(K_{n}\right)=n+1$ if $n$ is even, and $\chi^{T}\left(K_{n}\right)=n$ if $n$ is odd, and he presents a way to color $K_{n}$ in each case to demonstrate this. The total colorings he produces also happen to be optimal, if the colors are replaced by the first $\chi^{T}\left(K_{n}\right)$ natural numbers. First, note that the number of elements in $K_{n}$ is

$$
n+\left(\begin{array}{l}
n \\
2
\end{array}\right)=n+\frac{n(n-1)}{2}=\frac{2 n}{2}+\frac{n^{2}-n}{2}=\frac{n(n+1)}{2} .
$$

Case 1: $n$ is odd. Construct a total coloring $\phi$ of $K_{n}$ that uses only $\chi^{T}\left(K_{n}\right)=n$ colors. Each color class up to $n$ must have size $\alpha^{T}\left(K_{n}\right)=\frac{n+1}{2}$, because otherwise the number of elements colored would be less than $n\left(\frac{n+1}{2}\right)$, and that would not color all elements. Therefore, $\phi_{i}=\alpha^{T}$ for each color $i \leq n$, and $\phi$ is optimal by the First Corollary to Theorem 10. This implies $\sigma^{T}\left(K_{n}\right)=n=\chi^{T}\left(K_{n}\right)$, and $\Sigma^{T}\left(K_{n}\right)$ $=\operatorname{sum}(\phi)=\alpha^{T}+2 \alpha^{T}+\ldots+n \alpha^{T}=\left(\frac{n+1}{2}\right)\left(\frac{n(n+1)}{2}\right)$.

Case 2: $n$ is even. Construct a total coloring $\phi$ of $K_{n}$ that uses only $\chi^{T}\left(K_{n}\right)=n+1$ colors. Each color class up to $n+1$ must have size $\alpha^{T}\left(K_{n}\right)=\frac{n}{2}$, because otherwise the number of elements colored would be less than $(n+1)\left(\frac{n}{2}\right)$, and that would not color all elements. Therefore, $\phi_{i}=\alpha^{T}$ for each color $i \leq n+1$, and $\phi$ is optimal by the First Corollary to Theorem 10. This implies $\sigma^{T}\left(K_{n}\right)=n+1=\chi^{T}\left(K_{n}\right)$, and $\Sigma^{T}\left(K_{n}\right)=\operatorname{sum}(\phi)=\alpha^{T}+2 \alpha^{T}+\ldots+(n+1) \alpha^{T}=\left(\frac{n}{2}\right)\left(\frac{(n+1)(n+2)}{2}\right)$.

Theorem 15. For each pair of integers $m, n \geq 1$, it is true that $\sigma^{T}\left(K_{m, n}\right)=$ $\chi^{T}\left(K_{m, n}\right)$, and

$$
\Sigma^{T}\left(K_{m, n}\right)= \begin{cases}\frac{n(n+2)(n+3)}{2} & \text { if } m=n \\
(n-m)\left(\begin{array}{l}
j \\
2
\end{array}\right)+j(p-m)+m\left(\begin{array}{c}
n+1 \\
2
\end{array}\right) & \text { otherwise }\end{cases}
$$

where $j=\left\lceil\frac{n}{n-m}\right\rceil$ and $p=2 n-j(n-m)$. 
Proof. Let $K_{m, n}$ be a complete bipartite graph. Partition its vertices into the independent sets $M$ and $N$, such that $V\left(K_{m, n}\right)=M \cup N$ and $|M| \leq|N|$. Let $m=|M|$ and $n=|N|$. Then $\alpha^{T}=\alpha^{T}\left(K_{m, n}\right)=n$, by Theorem 5. Yap shows in [29] that $\chi^{T}\left(K_{m, n}\right)=n+2$ if $m=n$; otherwise it is $n+1$.

Case 1: $m=n$. Then $\chi^{T}\left(K_{n, n}\right)=n+2$. Construct a total coloring $\phi$ of $K_{n, n}$ that uses only $n+2$ colors. Each color class must have size $\alpha^{T}=n$, because otherwise the number of elements colored would be less than the number of elements in the graph, which is $2 n+n^{2}=n(n+2)$. Therefore, $\phi_{i}=\alpha^{T}$ for each color $i \leq n+2$, and $\phi$ is optimal by the First Corollary to Theorem 10. This implies $\sigma^{T}\left(K_{n, n}\right)=n+2$ $=\chi^{T}\left(K_{n, n}\right)$, and $\Sigma^{T}\left(K_{n, n}\right)=\operatorname{sum}(\phi)=\alpha^{T}+2 \alpha^{T}+\ldots+(n+2) \alpha^{T}=n\left(\frac{(n+2)(n+3)}{2}\right)$.

Case 2: $m<n$. Then $\chi^{T}\left(K_{m, n}\right)=n+1$. Denote the vertices in $M$ as $v_{1}, v_{2}$, etc., up to $v_{m}$. For each positive integer $i \leq m$, let $L_{i}$ be the subset of elements that includes $v_{i}$ and all edges incident to $v_{i}$ in $K_{m, n}$. Now let $\psi$ be an arbitrary coloring of $K_{m, n}$. Every vertex in $M$ has degree $n$, so for each positive integer $i \leq m$, there have to be $n+1$ elements in the set $L_{i}$. No two elements in $L_{i}$ can have the same color, so for each color $c \leq n+1$, there are at most $c$ elements in $L_{i}$ with color less than or equal to $c$, and likewise at least $n+1-c$ elements in $L_{i}$ with a color greater than $c$. Furthermore, there are $m$ mutually disjoint subsets $L_{i}$ in $K_{m, n}$, so in total, for each color $c \leq n+1=\chi^{T}\left(K_{m, n}\right)$, there are at least $m(n+1-c)$ elements in $K_{m, n}$ that are assigned a color greater than $c$ :

$$
\sum_{k>c} \psi_{k} \geq m\left(\chi^{T}-c\right)
$$

for every total coloring $\psi$ of $K_{m, n}$ and every color $c \leq \chi^{T}$. This is the exact property specified by Theorem 11, so if we can find a total coloring of $K_{m, n}$ that satisfies the other conditions in that theorem, we can claim that it is optimal. Create the coloring $\phi$ using the procedure below.

1. Denote the vertices in $N$ as $u_{0}, u_{1}$, etc., up to $u_{n-1}$. Let $t=n-m$. 
2. For each color $c \geq 1$, assign color $c$ to every vertex $u_{h} \in N$ such that $h \in$ $[(c-1) t, c t-1]$. The last color assigned this way is $\phi\left(u_{n}\right)=[n / t] \geq 2$.

3. For every color $c \in\left[1, \phi\left(u_{n}\right)-1\right]$, assign color $c$ to $u_{c t} v_{1}$ and assign color $\phi\left(u_{n}\right)$ to $u_{0} v_{1}$.

4. For every integer $h \in[0, n-1]$, in increasing order, if $u_{h} v_{1}$ is not yet colored, give it the least color not yet assigned to an edge incident to $v_{1}$.

5. Assign every vertex in $M$ color $n+1$.

6. Run the next step for every integer $i$ from 0 to $n-1$ :

7. Let $c$ be the color of edge $u_{i} v_{1}$. For every $j \in[2, m]$, let $k=i+j-1(\bmod n)$ and assign $u_{k} v_{j}$ color $c$.

We must now check to be sure that $\phi$ is a proper total-coloring.

- Every pair of adjacent vertices will involve one from $N$, which has color at most $\phi\left(u_{n}\right)<n$, and one from $M$, which has color $n+1$. Therefore, the coloring is proper with respect to vertices.

- In step 7, edge $u_{i} v_{1}$ has color $c$, and the only other edges that get color $c$ in the algorithm are those that receive it in step 7 . Here we can observe that no two edges with color $c$ meet at a common vertex, because values of $j$ and $k$ are unique as $j$ increases from 2 to $m$. Therefore, the coloring is proper with respect to edges.

- There are no $(n+1)$-colored edges, so no vertex-edge conflicts occur with that color. For colors $c \in\left[\phi\left(u_{n}\right)+1, n\right]$, there are no $c$-colored vertices, so no vertex-edge conflicts occur with that color. Color $\phi\left(u_{n}\right)$ only appears on edges incident to the first $m-1$ vertices of $N$, and that color does not appear 
on vertices of $N$ until index $([n / t]-1) t \geq n-t=m$, so no vertex-edge conflicts occur with color $\phi\left(u_{n}\right)$.

- For a color $c<\phi\left(u_{n}\right)$, observe that no $c$-colored edge can have an endvertex $u_{h} \in N$ with $h \in[c t-t, c t-1]$. (Edge $u_{c t} v_{1}$ gets color $c$ in step 3, but that index is outside this interval. Color $c$ is also assigned to edge $u_{k} v_{j}$ for certain values of $j$ and $k$ in step 7 , but $k$ starts at $c t+1$ and increases from there. Since $k$ is computed modulo $n$, it is reduced by $n$ when $j$ is high enough, but in that event, $k$ is at most $c t+m-1-n=c t-t-1$.) Fortunately, the only $c$-colored vertices in $N$ are those whose index lies inside the interval $[c t-t, c t-1]$, so no vertex-edge conflicts occur with this color.

Let $j=\phi_{u_{n}}-1$. This coloring assigns every color in $[1, j-1]$ to $t$ vertices and $m$ edges, for a total of $n$ elements, so $\phi_{k}=n=\alpha^{T}$ for all colors $k<j$. It assigns every color in $[j+1, n+1]$ to exactly $m$ elements, so $\phi_{k}=m$ for all colors in $\left[j, \chi^{T}\right]$. And it uses only $n+1$ colors, so $\phi_{k}=0$ for all $i>\chi^{T}$. By Theorem 11, $\phi$ is optimal.

This implies $\sigma^{T}\left(K_{m, n}\right)=n+1=\chi^{T}$. Also, $\Sigma^{T}\left(K_{m, n}\right)$

$$
\begin{aligned}
=\operatorname{sum}(\phi) & =\sum_{k=1}^{j-1} k n+j \phi_{j}+\sum_{k=j+1}^{n+1} k m \\
& =n\left(\frac{j(j-1)}{2}\right)+j \phi_{j}+m\left(\frac{(n+1)(n+2)}{2}-\frac{j(j+1)}{2}\right) \\
& =n\left(\begin{array}{l}
j \\
2
\end{array}\right)+j \phi_{j}+m\left(\begin{array}{c}
n+1 \\
2
\end{array}\right)-m\left(\frac{j(j-1)}{2}\right)-m\left(\frac{2 j}{2}\right) \\
& =(n-m)\left(\begin{array}{l}
j \\
2
\end{array}\right)+j\left(\phi_{j}-m\right)+m\left(\begin{array}{c}
n+1 \\
2
\end{array}\right), \\
\text { where } j & =\left[\begin{array}{c}
n \\
n-m
\end{array}\right] . \\
\text { and } \phi_{j} & =(n+m+n m)-n(j-1)-m(n+1-j) \\
& =2 n-j(n-m) .
\end{aligned}
$$




\subsection{Total Chromatic Sum of Some Hypercubes}

Theorem 16. If $Q_{n}$ is the hypercube of dimension $n$ and if $\alpha^{T}\left(Q_{n}\right)=5 \times 2^{n-3}$, then $\sigma^{T}\left(Q_{n}\right)=\chi^{T}\left(Q_{n}\right)$ and

$$
\Sigma^{T}\left(Q_{n}\right)=2^{d-2}\left(d^{2}+3 d+7\right) .
$$

Proof. Label each vertex according to its binary coordinate in $n$ dimensions, as it was done in Chapter 2. Let $A$ be the set of all vertices whose coordinate contains an even number of 1's. $A$ must be independent, because every pair of adjacent vertices in $Q_{n}$ differs in exactly one dimension, which makes the number of 1's in their coordinates differ by 1 , and thus have opposite parity. There should be an equal number of vertices whose coordinate contains an odd number of 1's, so $A$ is half the size of $V\left(Q_{n}\right):|A|=\frac{1}{2}\left(2^{n}\right)=2^{n-1}$.

Denote the vertices in $A$ arbitrarily as $v_{1}, v_{2}$, etc., up to $v_{m}$, where $m=|A|$. For each positive integer $i \leq m$, let $L_{i}$ be the subset of elements that includes $v_{i}$ and all edges incident to $v_{i}$ in $Q_{n}$. Now let $\psi$ be an arbitrary coloring of $Q_{n}$. Every vertex in $A$ has degree $n$, so for each positive integer $i \leq m$, there have to be $n+1$ elements in the set $L_{i}$. No two elements in $L_{i}$ can have the same color, so for each color $c \leq n+1$, there are at most $c$ elements in $L_{i}$ with color less than or equal to $c$, and likewise at least $n+1-c$ elements in $L_{i}$ with a color greater than $c$. Furthermore, there are $m=|A|$ mutually disjoint subsets $L_{i}$ in $Q_{n}$, so in total, for each color $c \leq n+1=\chi^{T}\left(Q_{n}\right)$, there are at least $m(n+1-c)$ elements in $Q_{n}$ that are assigned a color greater than $c$ :

$$
\sum_{k>c} \psi_{k} \geq m\left(\chi^{T}-c\right),
$$

for every total coloring $\psi$ of $Q_{n}$ and for every color $c \leq \chi^{T}\left(Q_{n}\right)$. This is the exact property specified by Theorem 11, so if we can find a total coloring of $Q_{n}$ that 
satisfies the other conditions in that theorem, we can claim that it is optimal. Create the coloring $\phi$ using the algorithm below.

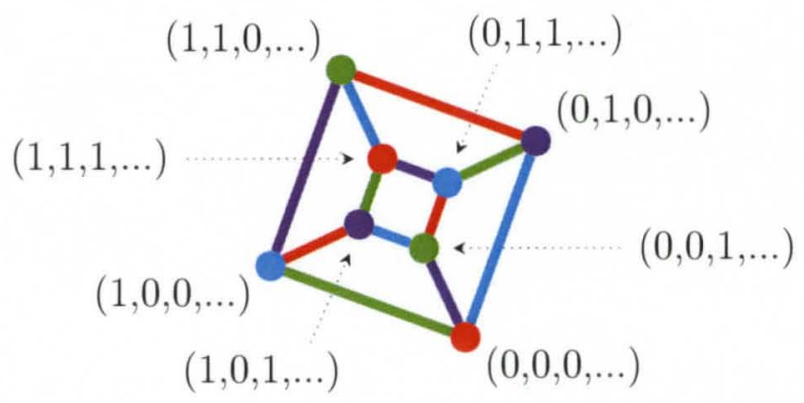

FIGURE 3.6: The total-4-coloring of a 3-cube subgraph of $Q_{n}$, given in step 1 of the algorithm. $($ Red $=1$, Blue $=2$, Green $=3$, and Purple $=4$.

1. Have $\phi$ assign colors 1 (red), 2 (blue), 3 (green), and 4 (purple) to the 20 elements shown in Figure 3.6, where in each coordinate, the entry equals 0 in every dimension greater than 3 .

2. Set the variable $d=4$.

3. Define the automorphism $r_{d}$ on $Q_{n}$ so that for each vertex $v \in Q_{n}, r_{d}(v)$ is the vertex in $Q_{n}$ whose coordinate is the same in all dimensions except 1 and $d$, where it is different. Let $C$ be the set of all vertices already colored at this moment; then every vertex in $C$ is equal to 0 in every dimension $d$. To each vertex $v \in C$, assign the vertex $r_{d}(v)$ the same color as $v$. To each edge $u v$ whose endvertices are in $C$, assign the edge $r_{d}(u) r_{d}(v)$ the same color as $u v$. (Justification for why this produces a proper coloring follows the same logic as the proof of Theorem 9, in which I explain why a union of a total independent set and its image via this automorphism retains its independence.)

4. If $d<n$, increase $d$ by 1 and return to the last step. Otherwise, proceed. 
5. Now all vertices and all edges that span dimensions 1,2 , and 3 have been given a color in $\{1,2,3,4\}$. For every other edge whose spanning dimension is $d \geq 4$, assign it the color $d+1$. Now, every color class $d \geq 4$ is a set of independent edges with size $2^{n-1}$ (the total number of edges divided by the number of dimensions).

The algorithm produces a proper total coloring $\phi$. In the first step, the first 4 colors were each assigned to 5 elements, and the number of elements having these colors doubled in every instance of step 3 . That step is repeated $n-3$ times, so for every color $c \leq 4, \phi_{c}=5\left(2^{n-3}\right)=\alpha^{T}\left(Q_{n}\right)$. For every color $c>4, c$ was assigned only to $2^{n-1}$ edges in step 5 , so $\phi_{c}=2^{n-1}=|A|=m$. Therefore, by Theorem $11, \phi$ is an optimal coloring of $Q_{n}$. It uses $n+1$ colors, so $\sigma^{T}\left(Q_{n}\right)=n+1=\chi^{T}\left(Q_{n}\right)$.

Also,

$$
\begin{aligned}
\Sigma^{T}\left(Q_{n}\right)=\operatorname{sum}(\phi) & =\sum_{d=1}^{4} d \alpha^{T}\left(Q_{n}\right)+\sum_{d=5}^{n+1} d m \\
& =\sum_{d=1}^{4} d\left(5 \times 2^{n-3}\right)+\sum_{d=5}^{n+1} d 2^{n-1} \\
& =\sum_{d=1}^{4} d\left(5 \times 2^{n-3}-2^{n-1}\right)+\sum_{d=1}^{n+1} d 2^{n-1} \\
& =2^{n-3} \sum_{d=1}^{4} d(5-4)+\frac{(n+1)(n+2)}{2}\left(2^{n-1}\right) \\
& =2^{n-3}(10)+2^{n-2}\left(n^{2}+3 n+2\right) \\
& =2^{n-2}\left(n^{2}+3 n+7\right) .
\end{aligned}
$$




\subsection{Total Chromatic Sum of Full Binary Trees}

For each $k \in \mathbb{N}$, define the following:

- Let $B_{k}$ be the full binary tree of height $k$, and let $r\left(B_{k}\right)$ denote its root vertex.

- The graph $B_{k}-r\left(B_{k}\right)$ has two components, each of which is is isomorphic to $B_{k-1}$. For each neighbor $u$ of $r\left(B_{k}\right)$, let $B_{k}(u)$ denote the component of $B_{k}-r\left(B_{k}\right)$ having root $u$.

- Define the function $W_{k}: \mathbb{N}^{3} \rightarrow \mathbb{N}$ as follows: Given the ordered 3-tuple $\left(c_{1}, c_{2}, c_{3}\right) \in \mathbb{N}^{3}$, with $c_{1}, c_{2}$, and $c_{3}$ all distinct, $W_{k}$ returns the smallest possible sum of a total coloring of $B_{k}$ in which $r\left(B_{k}\right)$ gets color $c_{1}$ and its incident edges get the colors $c_{2}$ and $c_{3}$ :

$$
W_{k}\left(c_{1}, c_{2}, c_{3}\right)=\min _{\phi \in \Phi\left(B_{k}\right)}\left\{\operatorname{sum}(\phi): \phi\left(r\left(B_{k}\right)\right)=c_{1}, \phi^{*}\left(r\left(B_{k}\right)\right)=\left\{c_{1}, c_{2}, c_{3}\right\}\right\} .
$$

Theorem 17. The following is true for each $n \in \mathbb{N}$ :

1. $\sigma^{T}\left(B_{n}\right)=\chi^{T}\left(B_{n}\right)$,

2. $\Sigma^{T}\left(B_{n}\right)=\frac{1}{3}\left(26 \times 2^{n}-24+(-1)^{n}\right)$, and

3. If $n$ is odd, every optimal coloring of $B_{n}$ must assign its root the color 3.

Proof. For each height $k \geq 1, \Sigma^{T}\left(B_{k}\right)$ is just the lowest possible value of $W_{k}$. In the case of $W_{1}$, given each possible coloration of $r\left(B_{1}\right)$ and its incident edges, we can determine the lowest possible colors for the neighbors of $v$, and add these colors together to determine the sum. Each row of Table 3.1 lists an element or interval of its domain, $N^{3}$, and taking those to be the colors of $r\left(B_{1}\right)$ and its incident edges, a function value of $W_{1}$ (or a lower bound for it) is generated. From the result, we can see that $W_{1}$ has a minimum of 9 , so $\Sigma^{T}\left(B_{1}\right)=9$. Note, also, that the 
total coloring of $B_{1}$ can only have sum 9 if $v$ has color 3. Figure 3.7 illustrates an optimal total-3-coloring of $B_{1}$, so $\sigma^{T}\left(B_{1}\right)=3=\Delta\left(B_{1}\right)+1=\chi^{T}\left(B_{1}\right)$. The theorem is true for $n=1$.

For $k>1$, we calculate $W_{k}\left(c_{1}, c_{2}, c_{3}\right)$ in three steps: (1) we find the lowest sum $s_{1}$ among all colorings of $B_{k-1}$ in which $r\left(B_{k-1}\right)$ does not have colors $c_{1}$ or $c_{2}$ and its incident edges do not have the color $c_{2} ;(2)$ we find the lowest sum $s_{2}$ among all colorings of $B_{k-1}$ in which $r\left(B_{k-1}\right)$ does not have colors $c_{1}$ or $c_{3}$ and its incident edges do not have the color $c_{3}$; and then (3) we calculate $c_{1}+c_{2}+c_{3}+s_{1}+s_{2}$. Steps (1) and (2) can be done by using the table of values for $W_{k-1}$.

\begin{tabular}{|l|l|l|c|c|c|}
\hline$c_{1}=$ & $c_{2}=$ & $c_{3}=$ & Least possible & Least possible & $W_{1}\left(c_{1}, c_{2}, c_{3}\right)$ \\
$\phi(v)$ & $\phi(v u)$ & $\phi(v w)$ & $\phi(u)$ & $\phi(w)$ & \\
\hline 1 & 2 & 3 & 3 & 2 & 11 \\
1 & 2 or 3 & 4 & $5-\phi(v u)$ & 2 & 12 \\
1 & $2^{+}$ & $5^{+}$ & $\geq 5-\phi(v u))$ & 2 & $13^{+}$ \\
\hline 2 & 1 & 3 & 3 & 1 & 10 \\
2 & 1 or 3 & 4 & $4-\phi(v u)$ & 1 & 11 \\
2 & $1^{+}$ & $5^{+}$ & $\geq 4-\phi(v u))$ & 1 & $12^{+}$ \\
\hline 3 & 1 & 2 & 2 & 1 & 9 \\
3 & 1 or 2 & 4 & $3-\phi(v u)$ & 1 & 11 \\
3 & $1^{+}$ & $5^{+}$ & $\geq 3-\phi(v u))$ & 1 & $12^{+}$ \\
\hline 4 & 1 & 2 & 2 & 1 & 10 \\
4 & 1 or 2 & 3 & $3-\phi(v u)$ & 1 & 11 \\
4 & $1^{+}$ & $5^{+}$ & $\geq 3-\phi(v u))$ & 2 & $13^{+}$ \\
\hline $5^{+}$ & $1^{+}$ & $2^{+}$ & $\geq 3-\phi(v u))$ & 1 & $11^{+}$ \\
\hline
\end{tabular}

TABLE 3.1: A table of values and lower bounds for $W_{1}$ using a graph $B_{1}$ having root vertex $v$ and the edges $v u$ and $v w$.

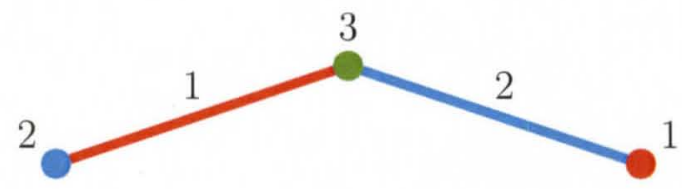

FIGURE 3.7: A total-3-coloring of $B_{1}$ with sum 9. 
Function values and lower bounds for $W_{2}$ and $W_{3}$ were found using this method, and are shown in Tables 3.2 and 3.3, respectively. From those tables we see that $W_{2}$ has a minimum of 27 , so $\Sigma^{T}\left(B_{2}\right)=27$, and $W_{3}$ has a minimum of 61 , so $\Sigma^{T}\left(B_{3}\right)=61$. The table shows that this optimal sum of $B_{3}$ is only possible if the root has color 3. Figures 3.8 and 3.9 illustrate optimal total-4-colorings of $B_{2}$ and $B_{3}$, so $\sigma^{T}\left(B_{2}\right)=4=\chi^{T}\left(B_{2}\right)$ and $\sigma^{T}\left(B_{3}\right)=4=\chi^{T}\left(B_{3}\right)$. Therefore, the theorem is true for $n=2$ and $n=3$.

\begin{tabular}{|l|l|l|c|c|c|}
\hline$c_{1}=$ & $c_{2}=$ & $c_{3}=$ & Least possible & Least possible & $W_{2}\left(c_{1}, c_{2}, c_{3}\right)$ \\
$\phi(v)$ & $\phi(v u)$ & $\phi(v w)$ & sum of $B_{2}(u)$ & sum of $B_{2}(w)$ & \\
\hline 1 & 2 & 3 & 11 & 10 & $\mathbf{2 7}$ \\
1 & 2 or 3 & 4 & $13-\phi(v u)$ & 9 & 27 \\
1 & $2^{+}$ & $5^{+}$ & $\geq 13-\phi(v u)$ & 9 & $28^{+}$ \\
\hline 2 & 1 & 3 or 4 & 11 & $13-\phi(v w)$ & 27 \\
2 & 3 & 4 & 10 & 9 & 28 \\
2 & $1^{+}$ & $5^{+}$ & $\geq 12-\phi(v u))$ & 9 & $28^{+}$ \\
\hline 3 & 1 & 2 & 11 & 11 & 28 \\
3 & 1 & 4 & 11 & 10 & 29 \\
3 & 2 & 4 & 11 & 10 & 30 \\
3 & $1^{+}$ & $5^{+}$ & $\geq 12-\phi(v u)$ & 10 & $30^{+}$ \\
\hline 4 & 1 or 2 & 2 or 3 & 11 & 11 & $29^{+}$ \\
4 & 1 or 2 & $5^{+}$ & 11 & 9 & $30^{+}$ \\
4 & $3^{+}$ & $5^{+}$ & $\geq 14-\phi(v u))$ & 9 & $32^{+}$ \\
\hline $5^{+}$ & 1 & $2^{+}$ & 11 & $\geq 13-\phi(v w)$ & $30^{+}$ \\
$5^{+}$ & $2^{+}$ & $3^{+}$ & $\geq 13-\phi(v u)$ & $\geq 13-\phi(v w)$ & $31^{+}$ \\
\hline
\end{tabular}

TABLE 3.2: A table of values and lower bounds for $W_{2}$ using a graph $B_{2}$ having root vertex $v$ and the edges $v u$ and $v w$.

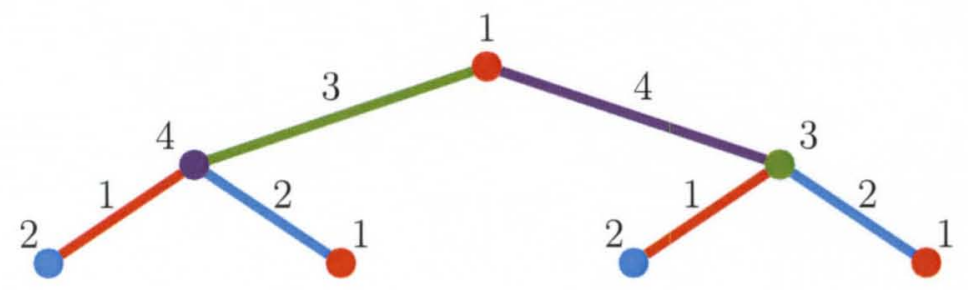

FIGURE 3.8: A total-4-coloring of $B_{2}$ with sum 27 . 


\begin{tabular}{|c|c|c|c|c|c|}
\hline $\begin{array}{l}c_{1}= \\
\phi(v)\end{array}$ & $\begin{array}{l}c_{2}= \\
\phi(v u)\end{array}$ & $\begin{array}{l}c_{3}= \\
\phi(v w)\end{array}$ & $\begin{array}{l}\text { Least possible } \\
\text { sum of } B_{3}(u)\end{array}$ & $\begin{array}{l}\text { Least possible } \\
\text { sum of } B_{3}(w)\end{array}$ & $W_{3}\left(c_{1}, c_{2}, c_{3}\right)$ \\
\hline 1 & 2 & 3 & 29 & 27 & 62 \\
\hline 1 & 2 & 4 & 29 & 27 & 63 \\
\hline 1 & 3 & 4 & 27 & 27 & 62 \\
\hline 1 & $2^{+}$ & $5^{+}$ & $\geq 30-\phi(v u)$ & 27 & $63^{+}$ \\
\hline 2 & 1 & 3 & 30 & 27 & 63 \\
\hline 2 & 3 & 4 & 27 & 27 & 63 \\
\hline 2 & $1^{+}$ & $4^{+}$ & 30 & 27 & $64^{+}$ \\
\hline 2 & $3^{+}$ & $5^{+}$ & 27 & 27 & $64^{+}$ \\
\hline 3 & 1 & 2 & 28 & 27 & 61 \\
\hline 3 & 1 & 4 & 28 & 27 & 63 \\
\hline 3 & 2 & 4 & 27 & 27 & 63 \\
\hline 3 & $1^{+}$ & $5^{+}$ & $\geq 29-\phi(v u)$ & 27 & $64^{+}$ \\
\hline 4 & 1 & 2 & 28 & 27 & 62 \\
\hline 4 & 1 or 2 & 3 & $29-\phi(v u)$ & 27 & 63 \\
\hline 4 & $1^{+}$ & $5^{+}$ & $\geq 29-\phi(v u))$ & 27 & $65^{+}$ \\
\hline $5^{+}$ & 1 & $2^{+}$ & 28 & 27 & $63^{+}$ \\
\hline $5^{+}$ & $2^{+}$ & $3^{+}$ & 27 & 27 & $64^{+}$ \\
\hline
\end{tabular}

TABLE 3.3: A table of values and lower bounds for $W_{3}$ using a graph $B_{3}$ having root vertex $v$ and the edges $v u$ and $v w$.

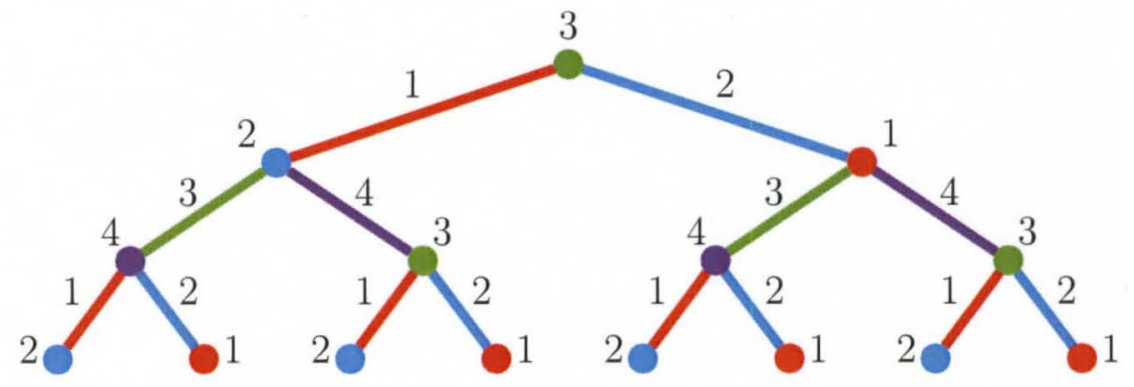

FIGURE 3.9: A total-4-coloring of $B_{3}$ with sum 61 . 
Function values for $W_{k}$ do not begin to show a recursive pattern until $k \geq 4$. The following proposition will be used to control function values of $W_{k}$ for these values. Though it has some advantages over Theorem 17, it is cumbersome, and is inaccurate for values of $k$ less than 4 . To simplify notation, define the sequence $\left\{a_{k}\right\}$ so that $a_{k}=\Sigma^{T}\left(B_{k}\right)$ for every non-negative integer $k$.

Proposition: For each $k \geq 4$, the following statements are true:

- The function values / lower bounds of $W_{k}$ are as shown in Table 3.4, as a function of $a_{k-1}$.

- If $k$ is even, then $a_{k}=2 a_{k-1}+9$ and:

1. A coloring $\phi$ of $B_{k}$ exists such that the root is assigned color 1, the root's incident edges are assigned colors 3 and $4, \operatorname{sum}(\phi)=a_{k}$, and $\sigma(\phi)=4$;

2. A coloring $\psi$ of $B_{k}$ exists such that the root is assigned color 2, the root's incident edges are assigned colors 3 and $4, \operatorname{sum}(\psi)=a_{k}+1$, and $\sigma(\psi)=4$.

- If $k$ is odd, then $a_{k}=2 a_{k-1}+7$ and:

1. Every coloring of $B_{k}$ with sum $a_{k}$ assigns the root the color 3;

2. A coloring $\phi$ of $B_{k}$ exists such that the root is assigned color 3, the root's incident edges are assigned colors 1 and $2, \operatorname{sum}(\phi)=a_{k}$, and $\sigma(\phi)=4$;

3. A coloring $\psi$ of $B_{k}$ exists such that the root is assigned color 4 , the root's incident edges are assigned colors 1 and $2, \operatorname{sum}(\psi)=a_{k}+1$, and $\sigma(\psi)=4$ 


\begin{tabular}{|l|l|l|c|c|}
\hline$c_{1}=$ & $c_{2}=$ & $c_{3}=$ & $W_{k}(\phi(v), \phi(v u), \phi(v w))$ & $\begin{array}{c}W_{k}(\phi(v), \phi(v u), \phi(v w)) \\
\text { when } k>4 \text { is odd }\end{array}$ \\
$\phi(v)$ & $\phi(v u)$ & $\phi(v w)$ & when $k \geq 4$ is even & $2 a_{k-1}+8$ \\
\hline 1 & 2 & 3 & $2 a_{k-1}+9$ & $2 a_{k-1}+9$ \\
1 & 2 & 4 & $2 a_{k-1}+9$ & $2 a_{k-1}+10^{+}$ \\
1 & 2 & $5^{+}$ & $2 a_{k-1}+10^{+}$ & $2 a_{k-1}+8$ \\
1 & 3 & 4 & $2 a_{k-1}+9$ & $2 a_{k-1}+9^{+}$ \\
1 & 3 & $5^{+}$ & $2 a_{k-1}+10^{+}$ & $2 a_{k-1}+10^{+}$ \\
1 & $4^{+}$ & $5^{+}$ & $2 a_{k-1}+10^{+}$ & $2 a_{k-1}+8$ \\
\hline 2 & 1 & 3 & $2 a_{k-1}+9$ & $2 a_{k-1}+9$ \\
2 & 1 & 4 & $2 a_{k-1}+9$ & $2 a_{k-1}+10^{+}$ \\
2 & 1 & $5^{+}$ & $2 a_{k-1}+10^{+}$ & $2 a_{k-1}+9$ \\
2 & 3 & 4 & $2 a_{k-1}+10$ & $2 a_{k-1}+10^{+}$ \\
2 & 3 & $5^{+}$ & $2 a_{k-1}+11^{+}$ & $2 a_{k-1}+11^{+}$ \\
2 & $4^{+}$ & $5^{+}$ & $2 a_{k-1}+11^{+}$ & $2 a_{k-1}+7$ \\
\hline 3 & 1 & 2 & $2 a_{k-1}+9$ & $2 a_{k-1}+9$ \\
3 & 1 & 4 & $2 a_{k-1}+11$ & $2 a_{k-1}+10^{+}$ \\
3 & 1 & $5^{+}$ & $2 a_{k-1}+12^{+}$ & $2 a_{k-1}+9$ \\
3 & 2 & 4 & $2 a_{k-1}+11$ & $2 a_{k-1}+10^{+}$ \\
3 & 2 & $5^{+}$ & $2 a_{k-1}+12^{+}$ & $2 a_{k-1}+12^{+}$ \\
3 & $4^{+}$ & $5^{+}$ & $2 a_{k-1}+14^{+}$ & $2 a_{k-1}+8$ \\
\hline 4 & 1 & 2 & $2 a_{k-1}+10$ & $2 a_{k-1}+9$ \\
4 & 1 & 3 & $2 a_{k-1}+12$ & $2 a_{k-1}+11^{+}$ \\
4 & 1 & $5^{+}$ & $2 a_{k-1}+12^{+}$ & $2 a_{k-1}+9$ \\
4 & 2 & 3 & $2 a_{k-1}+12$ & $2 a_{k-1}+11^{+}$ \\
4 & 2 & $5^{+}$ & $2 a_{k-1}+12^{+}$ & $2 a_{k-1}+12^{+}$ \\
4 & 3 & $5^{+}$ & $2 a_{k-1}+14^{+}$ & $2 a_{k-1}+15^{+}$ \\
4 & $5^{+}$ & $6^{+}$ & $2 a_{k-1}+15^{+}$ & $2 a_{k-1}+10^{+}$ \\
\hline $5^{+}$ & 1 & 2 & $2 a_{k-1}+11^{+}$ & $2 a_{k-1}+11^{+}$ \\
$5^{+}$ & 1 & 3 & $2 a_{k-1}+12^{+}$ & $2 a_{k-1}+10^{+}$ \\
$5^{+}$ & 1 & $4^{+}$ & $2 a_{k-1}+12^{+}$ & $2 a_{k-1}+11^{+}$ \\
$5^{+}$ & 2 & 3 & $2 a_{k-1}+12^{+}$ & $2 a_{k-1}+12^{+}$ \\
$5^{+}$ & 2 & $4^{+}$ & $2 a_{k-1}+12^{+}$ & $2 a_{k-1}+15^{+}$ \\
$5^{+}$ & 3 & $4^{+}$ & $2 a_{k-1}+13^{+}$ & \\
$5^{+}$ & $4^{+}$ & $6^{+}$ & $2 a_{k-1}+15^{+}$ & \\
\hline & & & & \\
\hline
\end{tabular}

TABLE 3.4: A table of values and lower bounds for $W_{k}$, with $k \geq 4$, using a graph $B_{k}$ having root vertex $v$ and the edges $v u$ and $v w$. 
This Proposition will be proved using induction on $k$.

In the base case, $k=4$. In Table 3.5, function values for $W_{4}$ are created the same way Tables 3.2 and 3.3 were created, except that in each row, beside the sum given for each $B_{3}$ subtree, it also includes a set of colors for that subtree's root and incident edges $\left\langle c_{1}, c_{2}, c_{3}\right\rangle$ that will bring about such a sum. Also, the function value or lower bound for $W_{4}$ is expressed in terms of $a_{k-1}$.

The values and lower bounds for $W_{k}$ match those in Table 3.4 where $k$ is even. The lowest possible sum for a total coloring of $B_{4}$ is $131=2 a_{3}+9$, so this is the value of $a_{4}$. A total-4-coloring $\phi$ of $B_{4}$ exists in which the root has color 1 and its incident edges have colors 3 and 4 , and $\operatorname{sum}(\phi)=a_{k}$, and $\sigma(\phi)=4$, as demonstrated in Figure 3.10. And a total-4-coloring $\psi$ of $B_{4}$ exists in which the root has color 2 and its incident edges have colors 3 and 4 , and $\operatorname{sum}(\psi)=a_{k}+1$, and $\sigma(\psi)=4$, which is the same as $\phi$ except for the color of the root vertex. Therefore, the Proposition is true for $k=4$.

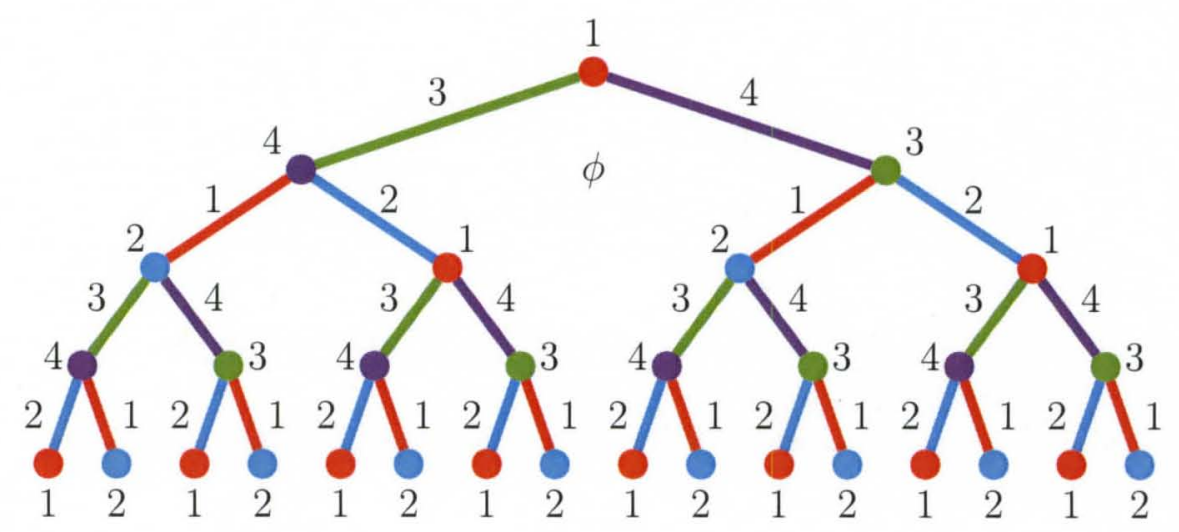

FIGURE 3.10: A total-4-coloring of $B_{4}$ with sum 131 . 


\begin{tabular}{|c|c|c|c|c|}
\hline $\begin{array}{l}\left\langle c_{1}, c_{2}, c_{3}\right\rangle= \\
\phi(\langle v, v u, v w\rangle)\end{array}$ & $\begin{array}{l}\text { Least sum } \\
\text { on } B_{4}(u)\end{array}$ & $\begin{array}{l}\text { Least sum } \\
\text { on } B_{4}(w)\end{array}$ & \multicolumn{2}{|c|}{$W_{4}(\phi(v), \phi(v u), \phi(v w))$} \\
\hline$<1,2,3>$ & $63:<3,1,4>$ & $62:\langle 4,1,2\rangle$ & $6+2 a_{3}+3$ & $=2 a_{3}+9$ \\
\hline & $3:<3,1,4>$ & $61:\langle 3,1,2\rangle$ & $7+2 a_{3}+2$ & $=2 a_{3}+9$ \\
\hline$<1,2$ & $3:<3$ & $51:<3,1,2>$ & $8++2 a+?$ & $=2 a_{3}+10^{+}$ \\
\hline & $62:\langle 4,1,2\rangle$ & $61:\langle 3,1,2\rangle$ & $8+2 a_{3}+1$ & $=2 a_{3}+9$ \\
\hline$<1,2$ & $62:\langle 4,1,2\rangle$ & $31:\langle 3,1,2\rangle$ & $0+2 a+1$ & $=2 a_{3}+10^{+}$ \\
\hline$<1$, & $61:<3$ & $61:<3$, & $10^{+}+2 a_{3}$ & $=2 a_{3}+10^{+}$ \\
\hline$<2,1$ & $3:<3,2,4>$ & $62:<4,1,2>$ & $6+2 a_{3}+3$ & $=2 a_{3}+9$ \\
\hline$<2$ & 2.2 & $2>$ & $7+2 a_{3}+2$ & $=2 a_{3}+9$ \\
\hline$<2,1,5^{+}>$ & $63:<3,2,4>$ & $61:\langle 3,1,2\rangle$ & $8^{+}+2 a_{3}+2$ & $=2 a_{3}+10^{+}$ \\
\hline$<2,3$ & $62:<4,1,2>$ & $61:<3,1,2>$ & $9+2 a_{3}+1$ & $=2 a_{3}+10$ \\
\hline$<2$, & $62:\langle 4,1,2>$ & $61:\langle 3,1,2\rangle$ & $10^{+}+2 a_{3}+1$ & $=2 a_{3}+11^{+}$ \\
\hline$<2,4$ & $61 . \quad 3$ & $61:<3,1,2>$ & $11+2$ & $=2 a_{3}+11^{+}$ \\
\hline$<3$ & $63:<2$, & $62:<1$ & $6+2 a_{3}+3$ & $=2 a_{3}+9$ \\
\hline$<3,1$ & $63:<2,3,4>$ & $62:\langle 1,2,3>$ & $8+2 a_{3}+3$ & $=2 a_{3}+11$ \\
\hline$<3$ & $63:<2$, & $62:<1$, & $5+2 a_{3}+5$ & $=2 a_{3}+12^{+}$ \\
\hline$<3$ & $62:<1,3,4>$ & $62:\langle 1,2,3>$ & $9+2 a_{3}+2$ & $=2 a_{3}+11$ \\
\hline$<3,2$ & $62:<1,3$ & $02 .<1$, & $10^{+}+2 a_{3}+2$ & $=2 a_{3}+12^{+}$ \\
\hline$<3$ & $62:<1,2$ & $62:<1$, & $12^{+}+2 a_{3}+2$ & $=2 a_{3}+14^{+}$ \\
\hline$<4$ & $63:<3,2,4>$ & $62:<1,3,4>$ & $7+2 a_{3}+3$ & $=2 a_{3}+10$ \\
\hline$<4$ & $63:<3$, & $63:\langle 1,2,4>$ & $8+2 a_{3}+4$ & $=2 a_{3}+12$ \\
\hline$<4$, & $63:\langle 3,2,4>$ & $61:<3,1,2>$ & $10^{+}+2 a_{3}+2$ & $=2 a_{3}+12^{+}$ \\
\hline$<4$ & $62:<1$ & $63:<1$, & $9+2 a_{3}+3$ & $=2 a_{3}+12$ \\
\hline$<4,2,5^{+}>$ & $62:<1,3,4>$ & $61:\langle 3,1,2\rangle$ & $11^{+}+2 a_{3}+1$ & $=2 a_{3}+12^{+}$ \\
\hline$<4,3,5^{+}>$ & $63:<1,2,4>$ & $61:<3,1,2>$ & $12^{+}+2 a_{3}+2$ & $=2 a_{3}+14^{+}$ \\
\hline$<4,5^{+}$ & $61:<3,1,2>$ & $61:\langle 3,1,2\rangle$ & $15^{+}+2 a_{3}$ & $=2 a_{3}+15^{+}$ \\
\hline$<5^{+}, 1,2>$ & $63:<2,3,4>$ & $62:<1,3,4>$ & $8^{+}+2 a_{3}+3$ & $=2 a_{3}+11^{+}$ \\
\hline$<5^{+}, 1,3>$ & $63:<2,3,4>$ & $62:<4,1,2>$ & $9^{+}+2 a_{3}+3$ & $=2 a_{3}+12^{+}$ \\
\hline$<5^{+}, 1,4^{+}>$ & $63:<2,3,4>$ & $61:\langle 3,1,2\rangle$ & $10^{+}+2 a_{3}+2$ & $=2 a_{3}+12^{+}$ \\
\hline$<5^{+}, 2,3>$ & $62:<1,3,4>$ & $62:\langle 4,1,2\rangle$ & $10^{+}+2 a_{3}+2$ & $=2 a_{3}+12^{+}$ \\
\hline$\left\langle 5^{+}, 2,4^{+}\right\rangle$ & $62:<1,3,4>$ & $61:<3,1,2>$ & $11^{+}+2 a_{3}+1$ & $=2 a_{3}+12^{+}$ \\
\hline$<5^{+}, 3,4^{+}>$ & $62:\langle 4,1,2\rangle$ & $61:\langle 3,1,2\rangle$ & $12^{+}+2 a_{3}+1$ & $=2 a_{3}+13^{+}$ \\
\hline$<5^{+}, 4^{+}, 6^{+}>$ & $61:\langle 3,1,2\rangle$ & $61:<3,1,2>$ & $15^{+}+2 a_{3}$ & $=2 a_{3}+15^{+}$ \\
\hline
\end{tabular}

TABLE 3.5: A table of values of $W_{k}$ in the basis case $(k=4)$, using a graph $B_{k}$ having root $v$ and the edges $v u$ and $v w$. The number 61 is always replaced with $a_{k-1}=a_{3}$ so that the resulting function value / lower bound of $W_{k}$ appears in the same form as it does in Table 3.4. 
Now for the induction step. Suppose $k>4$ and the Proposition is true for all integers less than $k$.

Case 1: $k$ is even.

By the induction hypothesis, the Proposition is true for $k-1$, which implies Table 3.4 correctly lists the function values and lower bounds of $W_{k-1}$, and also $a_{k-1}=2 a_{k-2}+7$. In Table 3.6, function values or lower bounds for $W_{k}$ are created using the odd column of Table 3.4 as a reference.

The results for $W_{k}$ match those in Table 3.4 where $k$ is even. The Table shows that the lowest possible sum for a total coloring of $B_{k}$ is $2 a_{k-1}+9$, so this is the value of $a_{k}$. Let $v=r\left(B_{k}\right)$ and let $v u, v w \in E\left(B_{k}\right)$.

A total-4-coloring $\phi$ of $B_{k}$ exists in which $\phi(v)=1, \phi(\{v u, v w\})=\{3,4\}$, and $\operatorname{sum}(\phi)=a_{k}$, which can be created in this way: Let $\left.\phi(\langle v, v u, v w\rangle)=<1,3,4\right\rangle$. The Proposition for $k-1$ states that we may give $B_{k}(u)$ a total-4-coloring where its root color is 4 and the edges of that subtree incident to the root are 1 and 2, for a total sum of $a_{k-1}+1$; color $B_{k}(u)$ this way in $\phi$. The Proposition also states that we may give $B_{k}(w)$ a total-4-coloring where its root color is 3 and the edges of that subtree incident to the root are 1 and 2 , for a total sum of $a_{k-1}$; color $B_{k}(w)$ this way in $\phi$. Then $\sigma(\phi)=4$, and $\operatorname{sum}(\phi)=1+3+4+2 a_{k-1}+1=2 a_{k-1}+9=a_{k}$.

Lastly, a total-4-coloring $\psi$ of $B_{k}$ exists in which $\phi(v)=1, \phi(\{v u, v w\})=$ $\{3,4\}$, and $\operatorname{sum}(\psi)=a_{k}+1$ : It can be created by copying $\phi$ and changing the root color $\psi(v)$ to 2 . Thus $\sigma(\psi)=4$ and $\operatorname{sum}(\psi)=\operatorname{sum}(\phi)-1+2=a_{k}+1$.

Therefore, the Proposition is true for this value of $k$. 


\begin{tabular}{|c|c|c|c|c|}
\hline $\begin{array}{l}\left\langle c_{1}, c_{2}, c_{3}\right\rangle= \\
\phi(\langle v, v u, v w\rangle)\end{array}$ & $\begin{array}{c}B_{k}(u) \text { lowest } \\
\text { sum: } 2 a_{k-2}+\ldots\end{array}$ & $\begin{array}{l}B_{k}(w) \text { lowest } \\
\text { sum: } 2 a_{k-2}+\ldots\end{array}$ & \multicolumn{2}{|c|}{$W_{k}(\phi(v), \phi(v u), \phi(v w))$} \\
\hline$<1,2,3>$ & $9:\langle 3,1,4\rangle$ & $8:\langle 4,1,2\rangle$ & $6+4 a_{k-1}+17$ & $=2 a_{k-1}+9$ \\
\hline$\langle 1,2,4\rangle$ & $9:\langle 3,1,4\rangle$ & $7:\langle 3,1,2\rangle$ & $7+4 a_{k-1}+16$ & \\
\hline & $9:\langle 3,1,4\rangle$ & $7:\langle 3,1,2\rangle$ & $8^{+}+4 a_{k-1}+16$ & $=2 a_{k-1}+10^{+}$ \\
\hline$<1,3$, & $8:\langle 4,1,2\rangle$ & $7:\langle 3,1,2\rangle$ & $+4 a_{k-1}+15$ & $=2 a_{k-1}+9$ \\
\hline$\left\langle 1,3,5^{+}\right\rangle$ & $8:\langle 4,1,2\rangle$ & $7:\langle 3,1,2\rangle$ & $9^{+}+4 a_{k-1}+15$ & $=2 a_{k-1}+10^{+}$ \\
\hline$<1,4$ & $7:<3,1$, & $7:<3,1$ & $10^{+}+4 a_{k-1}+14$ & $=2 a_{k-1}+10^{+}$ \\
\hline$<2$, & $9:\langle 3,2,4\rangle$ & $8:\langle 4,1,2\rangle$ & $6+4 a_{k-1}+17$ & $=2 a_{k-1}+9$ \\
\hline & $9:<3,2$ & $7:<3$, & $7+4 a_{k-1}+16$ & \\
\hline$\left\langle 2,1,5^{+}\right\rangle$ & $9:\langle 3,2,4>$ & $7:\langle 3,1,2\rangle$ & $8^{+}+4 a_{k-1}+16$ & $=2 a_{k-1}+10^{+}$ \\
\hline & $8:<4,1$ & $7:<3$, & $9+4 a_{k-1}+15$ & $=2 a_{k-1}+10$ \\
\hline$<2,3$ & $8:\langle 4,1,2\rangle$ & $7:\langle 3,1,2\rangle$ & $10^{+}+4 a_{k-1}+15$ & $=2 a_{k-1}+11^{+}$ \\
\hline$<2,4$ & $7:<3,1$, & $7:<3$ & $11^{+}+4 a_{k-1}+14$ & $=2 a_{k-1}+11^{+}$ \\
\hline$<3,1$ & $9:\langle 2,3,4\rangle$ & $8:\langle 1,3,4\rangle$ & $6+4 a_{k-1}+17$ & $=2 a_{k-1}+9$ \\
\hline$<3$ & $9:<2,3,4\rangle$ & $8:\langle 1,2,3\rangle$ & $8+4 a_{k-1}+17$ & $=2 a_{k-1}+11$ \\
\hline & $9:<2,3$ & $8:<1$, & $9^{+}+4 a_{k-1}+17$ & $=2 a_{k-1}+12^{+}$ \\
\hline$<3,2$ & $8:\langle 1,3,4>$ & $8:\langle 1,2,3\rangle$ & $9+4 a_{k-1}+16$ & $=2 a_{k-1}+11$ \\
\hline & $8:<1,3$ & $8:<1$, & & $=2 a_{k-1}+12^{+}$ \\
\hline$<3,4^{+}, 5^{+}>$ & $8:\langle 1,2,3\rangle$ & $8:\langle 1,2,3\rangle$ & $12^{+}+4 a_{k-1}+16$ & $=2 a_{k-1}+14^{+}$ \\
\hline$<4,1$ & $9:\langle 3,2,4\rangle$ & $8:<1,3$ & $7+4 a_{k-1}+17$ & $=2 a_{k-1}+10$ \\
\hline$<4,1$ & $9:\langle 3,2,4\rangle$ & $9:\langle 1,2,4\rangle$ & $8+4 a_{k-1}+18$ & $=2 a_{k-1}+12$ \\
\hline$<4,1$, & $9:\langle 3,2,4\rangle$ & $7:\langle 3,1,2\rangle$ & $10^{+}+4 a_{k-1}+16$ & $=2 a_{k-1}+12^{+}$ \\
\hline$<4,2$ & $8:<1,3$, & $9:<1$ & $9+4 a_{k-1}+17$ & $=2 a_{k-1}+12$ \\
\hline$<4,2$ & $8:\langle 1,3,4\rangle$ & $7:\langle 3,1,2\rangle$ & $11^{+}+4 a_{k-1}+15$ & $=2 a_{k-1}+12^{+}$ \\
\hline$<4,3$ & $9:<1,2$, & $7:\langle 3,1,2\rangle$ & $12^{+}+4 a_{k-1}+16$ & $=2 a_{k-1}+14^{+}$ \\
\hline$<4,5^{+}, 6^{+}>$ & $7:\langle 3,1,2\rangle$ & $7:\langle 3,1,2\rangle$ & $15^{+}+4 a_{k-1}+14$ & $=2 a_{k-1}+15^{+}$ \\
\hline$<5^{+}$, & $9:<2,3$, & $8:\langle 1,3,4\rangle$ & $8^{+}+4 a_{k-1}+17$ & $=2 a_{k-1}+11^{+}$ \\
\hline$<5^{+}, 1,3>$ & $9:\langle 2,3,4\rangle$ & $8:\langle 4,1,2\rangle$ & $9^{+}+4 a_{k-1}+17$ & $=2 a_{k-1}+12^{+}$ \\
\hline$\left.<5^{+}, 1,4^{+}\right\rangle$ & $9:\langle 2,3,4\rangle$ & $7:\langle 3,1,2\rangle$ & $10^{+}+4 a_{k-1}+16$ & $=2 a_{k-1}+12^{+}$ \\
\hline$<5^{+}, 2,3>$ & $8:\langle 1,3,4\rangle$ & $8:\langle 4,1,2\rangle$ & $10^{+}+4 a_{k-1}+16$ & $=2 a_{k-1}+12^{+}$ \\
\hline$\left\langle 5^{+}, 2,4^{+}>\right.$ & $8:\langle 1,3,4\rangle$ & $7:\langle 3,1,2\rangle$ & $11^{+}+4 a_{k-1}+15$ & $=2 a_{k-1}+12^{+}$ \\
\hline$<5^{+}, 3,4^{+}>$ & $8:<4,1,2\rangle$ & $7:\langle 3,1,2\rangle$ & $12^{+}+4 a_{k-1}+15$ & $=2 a_{k-1}+13^{+}$ \\
\hline$\left\langle 5^{+}, 4^{+}, 6^{+}\right\rangle$ & $7:\langle 3,1,2\rangle$ & $7:<3,1,2>$ & $15^{+}+4 a_{k-1}+14$ & $=2 a_{k-1}+15^{+}$ \\
\hline
\end{tabular}

TABLE 3.6: Evidence for Table 3.4 when $k>4$ is even, using a graph $B_{k}$ having root vertex $v$ and the edges $v u$ and $v w$. The relationship $a_{k-1}=2 a_{k-2}+7$ allows us to express the sum in terms of $a_{k-1}$. 


\begin{tabular}{|c|c|c|c|c|}
\hline $\begin{array}{c}\left\langle c_{1}, c_{2}, c_{3}\right\rangle= \\
\phi(\langle v, v u, v w\rangle)\end{array}$ & $\begin{array}{c}B_{k}(u) \text { lowest } \\
\text { sum: } 2 a_{k-2}+\ldots\end{array}$ & $\begin{array}{l}B_{k}(w) \text { lowest } \\
\text { sum: } 2 a_{k-2}+\ldots\end{array}$ & \multicolumn{2}{|c|}{$W_{k}(\phi(v), \phi(v u), \phi(v w))$} \\
\hline$<1,2,3>$ & $11:\langle 3,1,4\rangle$ & $9:\langle 2,1,4\rangle$ & $6+4 a_{k-1}+20$ & $=2 a_{k-1}+8$ \\
\hline$\langle 1,2,4\rangle$ & $11:\langle 3,1,4\rangle$ & $9:\langle 3,1,2\rangle$ & $7+4 a_{k-1}+20$ & $=2 a_{k-1}+9$ \\
\hline$<1,2$, & $11:\langle 3,1,4\rangle$ & $9:\langle 3,1,2\rangle$ & $8^{+}+4 a_{k-1}+20$ & $=2 a_{k-1}+10^{+}$ \\
\hline$<1,3$, & $9:\langle 2,1,4\rangle$ & $9:<3,1,2\rangle$ & $8+4 a_{k-1}+18$ & $=2 a_{k-1}+8$ \\
\hline$<1,3,5^{+}>$ & $9:\langle 2,1,4\rangle$ & $9:\langle 3,1,2\rangle$ & $9^{+}+4 a_{k-1}+18$ & $=2 a_{k-1}+9^{+}$ \\
\hline$<1$, & $9:<3,1$ & $9:\langle 3,1,2\rangle$ & $0^{+}+4 a_{k-1}+18$ & $=2 a_{k-1}+10^{+}$ \\
\hline$<2,1,3>$ & $11:<3,2,4\rangle$ & $9:\langle 1,2,4\rangle$ & $+4 a_{k-1}+20$ & $=2 a_{k-1}+8$ \\
\hline$<2,1$, & $11:<3,2$, & $9:<3,1,2>$ & $+4 a_{k-1}+20$ & $=2 a_{k-1}+9$ \\
\hline$\left\langle 2,1,5^{+}>\right.$ & $11:\langle 3,2,4\rangle$ & $9:\langle 3,1,2\rangle$ & $8^{+}+4 a_{k-1}+20$ & $=2 a_{k-1}+10^{+}$ \\
\hline$<2$, & $9:\langle 1,2,4\rangle$ & $9:\langle 3,1,2\rangle$ & $9+4 a_{k-1}+18$ & $=2 a_{k-1}+9$ \\
\hline$<2,3$ & $9:\langle 1,2,4\rangle$ & $9:\langle 3,1,2\rangle$ & $10^{+}+4 a_{k-1}+18$ & $=2 a_{k-1}+10^{+}$ \\
\hline$<2,4$ & $9:<1,2$ & $9:\langle 3,1,2\rangle$ & $11^{+}+4 a_{k-1}+18$ & $=2 a_{k-1}+11^{+}$ \\
\hline$<3$ & $10:<2,3,4\rangle$ & $9:\langle 1,2,3\rangle$ & $6+4 a_{k-1}+19$ & $=2 a_{k-1}+7$ \\
\hline$<3$, & $10:\langle 2,3,4\rangle$ & $9:\langle 1,2,3\rangle$ & $+4 a_{k-1}+19$ & $=2 a_{k-1}+9$ \\
\hline & $10:<2$, & $9:<1,2$ & & \\
\hline$<3$ & $9:\langle 1,3,4\rangle$ & $9:\langle 1,2,3\rangle$ & $9+4 a_{k-1}+18$ & $=2 a_{k-1}+9$ \\
\hline & $9:<1$ & $9:\langle 1,2,3\rangle$ & $10^{+}+4 a_{k-1}+18$ & $=2 a_{k-1}+10^{+}$ \\
\hline$<3,4^{+}, 5^{+}>$ & $9:\langle 1,2,3\rangle$ & $9:\langle 1,2,3\rangle$ & $12^{+}+4 a_{k-1}+18$ & $=2 a_{k-1}+12^{+}$ \\
\hline$<4$, & $10:<2,3$ & $9:<1,3,4\rangle$ & $7+4 a_{k-1}+19$ & $=2 a_{k-1}+8$ \\
\hline$<4$, & $10:<2$ & $9:\langle 1,2,4\rangle$ & $8+4 a_{k-1}+19$ & $=2 a_{k-1}+9$ \\
\hline$\left\langle 4,1,5^{+}\right\rangle$ & $10:\langle 2,3,4\rangle$ & $9:\langle 3,1,2\rangle$ & $10^{+}+4 a_{k-1}+19$ & $=2 a_{k-1}+11^{+}$ \\
\hline$<4,2,3\rangle$ & $9:<1,3$ & $9:\langle 1,2,4\rangle$ & $9+4 a_{k-1}+18$ & $=2 a_{k-1}+9$ \\
\hline$\left\langle 4,2,5^{+}\right\rangle$ & $9:\langle 1,3,4\rangle$ & $9:\langle 3,1,2\rangle$ & $11^{+}+4 a_{k-1}+18$ & $=2 a_{k-1}+11^{+}$ \\
\hline$<4,3$ & $9:<1,2$ & $9:<3,1$ & $12^{+}+4 a_{k-1}+18$ & $=2 a_{k-1}+12^{+}$ \\
\hline$<4,5^{+}, 6^{+}>$ & $9:\langle 3,1,2\rangle$ & $9:\langle 3,1,2\rangle$ & $15^{+}+4 a_{k-1}+18$ & $=2 a_{k-1}+15^{+}$ \\
\hline$\left\langle 5^{+}, 1,2>\right.$ & $10:<2,3$ & $9:<1,3$, & $8^{+}+4 a_{k-1}+19$ & $=2 a_{k-1}+9^{+}$ \\
\hline$\left.<5^{+}, 1,3\right\rangle$ & $10:\langle 2,3,4\rangle$ & $9:\langle 1,2,4\rangle$ & $9^{+}+4 a_{k-1}+19$ & $=2 a_{k-1}+10^{+}$ \\
\hline$<5^{+}, 1,4^{+}>$ & $10:\langle 2,3,4\rangle$ & $9:\langle 3,1,2\rangle$ & $10^{+}+4 a_{k-1}+19$ & $=2 a_{k-1}+11^{+}$ \\
\hline$<5^{+}, 2,3>$ & $9:\langle 1,3,4\rangle$ & $9:\langle 1,2,4\rangle$ & $10^{+}+4 a_{k-1}+18$ & $=2 a_{k-1}+10^{+}$ \\
\hline$<5^{+}, 2,4^{+}>$ & $9:\langle 1,3,4\rangle$ & $9:\langle 3,1,2\rangle$ & $11^{+}+4 a_{k-1}+18$ & $=2 a_{k-1}+11^{+}$ \\
\hline$\left\langle 5^{+}, 3,4^{+}>\right.$ & $9:\langle 1,2,4\rangle$ & $9:\langle 3,1,2\rangle$ & $12^{+}+4 a_{k-1}+18$ & $=2 a_{k-1}+12^{+}$ \\
\hline$\left\langle 5^{+}, 4^{+}, 6^{+}\right\rangle$ & $9:\langle 3,1,2\rangle$ & $9:\langle 3,1,2\rangle$ & $15^{+}+4 a_{k-1}+18$ & $=2 a_{k-1}+15^{+}$ \\
\hline
\end{tabular}

TABLE 3.7: Evidence for Table 3.4 when $k>4$ is odd, using a graph $B_{k}$ having root vertex $v$ and the edges $v u$ and $v w$. The relationship $a_{k-1}=2 a_{k-2}+9$ allows us to express the sum in terms of $a_{n-1}$. 
Case 2: $n$ is odd.

By the induction hypothesis, the Proposition is true for $k-1$, which implies Table 3.4 correctly lists the function values and lower bounds of $W_{k-1}$, and also $a_{k-1}=2 a_{k-2}+9$. In Table 3.6, function values or lower bounds for $W_{k}$ are created using the even column of Table 3.4 as a reference.

The results for $W_{k}$ match those in Table 3.4 where $k$ is odd. The Table shows that the lowest possible sum for a total coloring of $B_{k}$ is $2 a_{k-1}+7$, so this is the value of $a_{k}$. Furthermore, this sum can only be obtained if the root of $B_{k}$ is assigned color 3. Let $v=r\left(B_{k}\right)$ and let $v u, v w \in E\left(B_{k}\right)$.

A total-4-coloring $\phi$ of $B_{k}$ exists in which $\phi(v)=3, \phi(\{v u, v w\})=\{1,2\}$, and $\operatorname{sum}(\phi)=a_{k}$, which can be created in this way: Let $\phi(<v, v u, v w>)=<3,1,2>$. The Proposition for $k-1$ states that we may give $B_{k}(u)$ a total-4-coloring where its root color is 2 and the edges of that subtree incident to the root are 3 and 4 , for a total sum of $a_{k-1}+1$; color $B_{k}(u)$ this way in $\phi$. The Proposition also states that we may give $B_{k}(w)$ a total-4-coloring where its root color is 1 and the edges of that subtree incident to the root are 3 and 4 , for a total sum of $a_{k-1}$; color $B_{k}(w)$ this way in $\phi$. Then $\sigma(\phi)=4$, and $\operatorname{sum}(\phi)=3+1+2+2 a_{k-1}+1=2 a_{k-1}+7=a_{k}$.

Lastly, a total-4-coloring $\psi$ of $B_{k}$ exists in which $\phi(v)=4, \phi(\{v u, v w\})=$ $\{1,2\}$, and $\operatorname{sum}(\psi)=a_{k}+1$ : It can be created by copying $\phi$ and changing the root color $\psi(v)$ to 4 . Thus $\sigma(\psi)=4$ and $\operatorname{sum}(\psi)=\operatorname{sum}(\phi)-3+4=a_{k}+1$.

Therefore, the Proposition is true for both even and odd values of $k \geq 4$. Then, for each $k \geq 4$, a total-4-coloring of $B_{k}$ exists with sum $\Sigma^{T}\left(B_{k}\right)=a_{k}$, which implies $\sigma^{T}\left(B_{k}\right)=\chi^{T}\left(B_{k}\right)$. This was proven earlier for $1 \leq k \leq 3$, so the first part of the theorem must be true for every natural number.

The Proposition also implies that for each $k \geq 4$, the quantity $\Sigma^{T}\left(B_{k}\right)$ has 
the value $a_{k}$ described by the recurrence relation

$$
a_{k}= \begin{cases}2 a_{k-1}+9 & \text { if } k \text { is even, } \\ 2 a_{k-1}+7 & \text { if } k \text { is odd }\end{cases}
$$

We can see that $a_{0}=\Sigma^{T}\left(B_{0}\right)=1$ and that this recurrence relation holds for $1 \leq k \leq 3$ as well as $k \geq 4: a_{1}=2(1)+7=9, a_{2}=2(9)+9=27$, and $a_{3}=2(27)+7=61$. Thus we can obtain a closed formula for $\Sigma^{T}\left(B_{k}\right)$.

For even $k \geq 2, a_{k}=2 a_{k-1}+9=2\left(2 a_{k-2}+7\right)+9=4 a_{k-2}+23 ;$ solving for the homogeneous part, we obtain the general solution

$$
a_{k}=\lambda_{1} 2^{k}+\lambda_{2}(-2)^{k}+c=\lambda 2^{k}+c .
$$

Using the initial conditions $a_{0}=1$ and $a_{2}=27$, we get the system:

$$
\left\{\begin{array}{l}
1=\lambda+c \\
27=4 \lambda+c
\end{array} \quad \text { with the solutions } \lambda=\frac{26}{3}, c=\frac{-23}{3} .\right.
$$

This yields the particular solution $a_{k}=\frac{26}{3} 2^{k}-\frac{23}{3}$.

For odd $k \geq 3, a_{k}=2 a_{k-1}+7=2\left(2 a_{k-2}+9\right)+7=4 a_{k-2}+25 ;$ solving for the homogeneous part, we obtain the same general solution. Using the initial conditions $a_{1}=9$ and $a_{3}=61$, we get the system:

$$
\left\{\begin{array}{l}
9=2 \lambda+c \\
61=8 \lambda+c
\end{array} \quad \text { with the solutions } \lambda=\frac{26}{3}, c=\frac{-25}{3} .\right.
$$

This yields the particular solution $a_{k}=\frac{26}{3} 2^{k}-\frac{25}{3}$. Therefore, for each $k \geq 1$,

$$
\begin{aligned}
a_{k} & =\frac{26}{3} 2^{k}-\frac{24}{3}+\frac{(-1)^{k}}{3} \\
\Sigma^{T}\left(B_{k}\right) & =\frac{1}{3}\left(26 \times 2^{k}-24+(-1)^{k}\right) .
\end{aligned}
$$

Therefore, the second part of the theorem is true for every natural number. The third part of the theorem is directly implied by the Proposition and by the observations made in the $k=3$ case. 


\subsection{When No Optimal Total Coloring Maximizes $\phi_{1}$}

It is not surprising that the sequences for the total chromatic sum and total independence number of full binary trees would increase by a little more than a factor of two at every step, because at every step the graph is being cloned and joined to its copy via a new vertex. But it seems strange that the sequence $\left\{\Sigma^{T}\left(B_{n}\right)\right\}$ would jump at every even value of $n$, while Theorem 7 shows that the sequence $\left\{\alpha^{T}\left(B_{n}\right)\right\}$ jumps whenever $n$ is 0 or 2 modulo 3 . This disparity suggests that the total chromatic sum does not, in some sense, take advantage of the increasing total independence number. It turns out that, in the case of $B_{3}$, and for each full binary tree of height equal to $3(\bmod 6)$, no optimal total coloring can have a maximized first color class:

Theorem 18. For each $n \equiv 3(\bmod 6)$, if $\phi$ is an optimal total coloring of $B_{n}$, then $\phi_{1}<\alpha^{T}\left(B_{n}\right)$.

Proof. Let $n \in \mathbb{N}$ be a number s.t. $n \equiv 3(\bmod 6)$. Let $\phi$ be an optimal total coloring of $B_{n}$, and let $r$ be the root of $B_{n}$. Since $n$ is odd and $\phi$ is optimal, Theorem 17 dictates that $\phi(r)=3$. However, $n \equiv 0(\bmod 3)$, so Theorem 7 dictates that every total independent set of maximum size in $B_{n}$ must include $r$. Since $r$ has color 3, it is not in the first color class, so the first color class does not have maximum size. Therefore, $\phi_{1}<\alpha^{T}\left(B_{n}\right)$. 


\section{CHAPTER 4 THE T-STRENGTH OF GRAPHS}

\subsection{Total Strength vs. Total Chromatic Number}

In the previous chapter, an allusion was made to a strange coloring sum phenomenon present in some graphs: that is, a graph's strength can be more than its chromatic number, and a graph's edge strength can be more than its edge chromatic number. Here, the phenomenon will be studied for total colorings, and it will be given a name. We will say that a graph $G$ is T-strong if $\sigma^{T}(G)>\chi^{T}(G)$.

Theorems 12 to 16 in Chapter 3 addressed the total chromatic sum of some common graphs as well as the total strength of those graphs. Those families of graphs, including paths, cycles, complete graphs, complete bipartite graphs, full binary trees, and hypercubes up to dimension 6 , have something in common: Their total strength is equal to their total chromatic number; therefore, none of those graphs are $T$-strong. It is also true that stars are not $T$-strong, which follows from the next theorem.

\subsection{Trees of Maximum Distance Four or Less}

Theorem 19. There is no T-strong tree with maximum distance less than 5.

Proof. Suppose the theorem is false, and let $G$ be a $T$-strong tree having maximum distance 4 or less. With this property, the graph $G$ has a central vertex $s$ such that 
$\operatorname{dist}(v, s) \leq 2$ for every vertex $v \in V(G)$. Every edge incident to $s$ will be called an inner edge, and every non-leaf neighbor of $s$ will be called a hub; see Figure 4.1 for an example of this labeling.

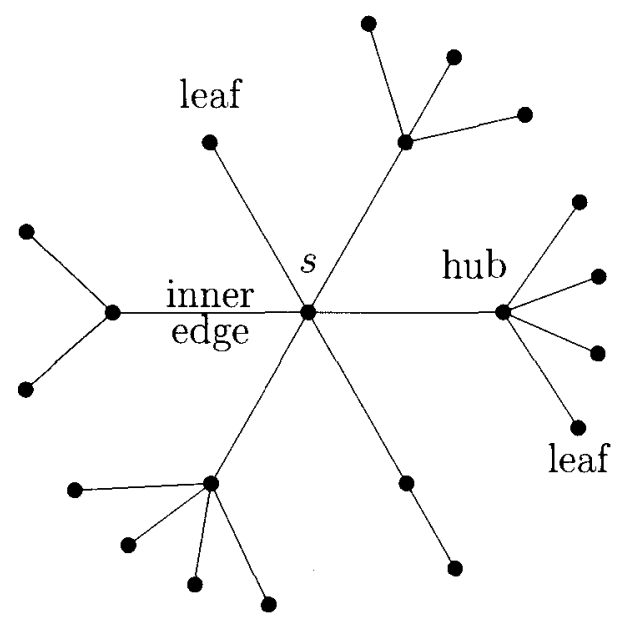

FIGURE 4.1: An example of a tree with maximum distance less than 5.

We now expand the definition of the distance metric dist for any pair of elements in the tree $G$ as follows. (It is tedious, but not difficult, to show that this is a true metric in any tree; the proof is omitted.) For any pair of elements $x, y \in V(G) \cup E(G)$, the distance $\operatorname{dist}(x, y)$ depends on whether $x$ and $y$ are vertices, edges, or one of each.

- If $x, y \in V(G)$, then distance is defined as normal.

- If $x, y \in E(G)$, let $x=a b$ and let $y=c d$; then

$$
\operatorname{dist}(x, y)=\max \{\operatorname{dist}(a, b), \operatorname{dist}(a, d), \operatorname{dist}(b, c), \operatorname{dist}(b, d)\}-1 \text {. }
$$

- If $x \in V(G)$ and $y \in E(G)$, let $y=c d$; then

$$
\operatorname{dist}(x, y)=\max \{\operatorname{dist}(x, c), \operatorname{dist}(x, d)\}-\frac{1}{2} .
$$


Let $\Delta=\Delta(G)$. Trees are Class 1 graphs, so $\chi^{T}(G)=\Delta+1$. Since $G$ is $T$-strong, every optimal total coloring of $G$ must require at least $\chi^{T}+1=\Delta+2$ colors. Define $\Phi_{\text {opt }} \subset \Phi(G)$ to be the set of all optimal colorings of $G$.

Define the function $h: \Phi_{\text {opt }} \rightarrow \mathbb{Z}$ to return the number of elements that are assigned a color $(\Delta+2)$ or higher in an optimal coloring $\psi$ :

$$
h(\psi)=\sum_{k \geq \Delta+2} \psi_{k} .
$$

Find the smallest value of $h$ among all colorings in $\Phi_{\text {opt }}$, and define $\Phi_{\text {few }} \subseteq \Phi_{\text {opt }}$ to be the set of optimal colorings that minimize $h$ within $\Phi_{o p t}$.

Define the function $g: \Phi_{\text {few }} \rightarrow \mathbb{Z}$ to return greatest distance between $s$ and an element of color $(\Delta+2)$ or more:

$$
g(\psi)=\max _{x \in V(G) \cup E(G)}\{\operatorname{dist}(x, s): \psi(x) \geq(\Delta+2)\} .
$$

Find the greatest value of $g$ among all colorings in $\Phi_{f e w}$, and define $\Phi_{f a r} \subseteq \Phi_{f e w}$ to be the set of colorings that maximize $g$ :

$$
\phi \in \Phi_{f a r} \subseteq \Phi_{f e w} \subseteq \Phi_{\text {opt }} \subset \Phi(G) .
$$

Now choose a coloring $\phi \in \Phi_{f a r}$. For any vertex $v$ and any total coloring $\phi$, we define function $\phi^{*}(v)$ to be the set of colors that $\phi$ assigns to $v$ and its incident edges, and define the function $\phi^{* *}(v)$ to be the set of colors that $\phi$ assigns to $v$, its incident edges and its adjacent vertices.

To derive a contradiction, our goal is to find a total coloring $\psi \neq \phi$ which has one of the following properties:

- $\psi$ is lighter than $\phi$, which means $\operatorname{sum}(\psi)<\operatorname{sum}(\phi)$, contradicting the assertion $\phi \in \Phi_{\text {opt }}$;

- $\psi$ is sparser than $\phi$, which means $\operatorname{sum}(\psi)=\operatorname{sum}(\phi)$ but $h(\psi)<h(\phi)$, contradicting the assertion $\phi \in \Phi_{\text {few }}$, or 
- $\psi$ is weaker than $\phi$, which means $\operatorname{sum}(\psi)=\operatorname{sum}(\phi)$ and $h(\psi)=h(\phi)$, but $g(\psi)>g(\phi)$, contradicting the assertion $\phi \in \Phi_{f a r}$.

Choose an element $x \in V(G) \cup E(G)$ such that $\phi(x) \geq(\Delta+2)$ and $\operatorname{dist}(x, s)=$ $g(\psi)$. No matter what type of element $x$ is, we will obtain a contradiction.

Case 1: $x=s$. Let $A$ be the set of all elements incident or adjacent to $x$. If a color $k \leq \Delta+1$ exists such that $k$ is assigned to only one element in $A$, then switch the colors on $x$ and that element to create a weaker coloring $\psi$. Assume, instead, that the color of every element in $A$ is assigned to at least two elements in $A$. Since $\operatorname{deg}(x) \leq \Delta$, we have $|A| \leq 2 \Delta$, so there are at most $\Delta$ colors assigned to the elements in $A$. Then we can reduce the color of $x$ to a color in $\{1, \ldots, \Delta+1\} \backslash \phi^{* *}(x)$ to create a lighter coloring $\psi$.

Case 2: $\quad x$ is an inner edge $s r$. Choose a color $k \in\{1, \ldots, \Delta+1\} \backslash \phi^{*}(s)$. If $k$ is assigned to $r$ or a pendant edge incident to $r$, switch the colors on $x$ and this $k$-colored element to create a weaker coloring $\psi$. Otherwise, $k \notin \phi^{\star}(r)$, so reduce the color of $x$ to $k$ to create a lighter coloring $\psi$.

Case 3: $\quad x$ is a pendant edge $r v$, from a hub $r$ to a leaf $v$. Choose a color $k \in\{1, \ldots, \Delta+1\} \backslash \phi^{*}(r)$. If $k \neq \phi(v)$, we can reduce the color of $r v$ to $k$ to create a lighter coloring $\psi$. Assume, instead, that $k=\phi(v)$. If $k>2$, we can reduce the color of $v$ to a color in $\{1,2\} \backslash \phi(r)$ to create a lighter coloring $\psi$. Otherwise, $k \leq 2$, so reduce the color of $r v$ to $k$ and reassign $v$ a color in $\{1,2,3\} \backslash\{k, \phi(r)\}$ to create a coloring $\psi$ which is lighter becase $\operatorname{sum}(\psi)-\operatorname{sum}(\phi)=($ change in $v$ 's color $)+$ (change in vr's color) $\leq 3-k+k-(\Delta+2)=1-\Delta<0$.

Case 4: $x$ is a leaf. Reduce its color to one in $\{1,2,3\} \backslash \phi^{*}(x)$ to create a lighter coloring $\psi$.

Case 5: $x$ is a hub of degree 4 or higher. If $x$ has a leaf neighbor $v$ whose color is greater than 2 , then we can reassign that leaf a color in $\{1,2\} \backslash \phi(x v)$ to create a 
lighter coloring $\psi$. If $x$ has two leaf neighbors with color 2 , then one of them, has an incident edge with color greater than 2 , and we can reduce that leaf's color to 1 to create a lighter coloring $\psi$. Assume, then, that every leaf adjacent to $x$ has a color in $\{1,2\}$, and at most one has color 2 .

If $\operatorname{deg}(x) \geq 5$, then $x$ has at least four incident pendant edges. Those edges must be assigned different colors, so at least two of them must be assigned a color greater than 2; let $k$ be a color that is assigned to a pendant edge incident to $x$, greater than 2 , and not equal to $\phi(s)$. Switch the colors on $x$ and that $k$-colored edge to create a weaker coloring $\psi$.

Assume, instead, that $\operatorname{deg}(x)=4$. Then $x$ has three leaf neighbors; let $C$ be the set of three colors assigned to the pendant edges incident to $x$. If $\phi(s) \notin \phi^{*}(x)$, then choose a color $k \in \phi^{*}(x) \backslash\{1,2, \phi(x s)\}$ and switch the colors on $x$ and the $k$-colored pendant edge to create a weaker coloring $\psi$. Assume, instead, that $\phi(s) \in \phi^{*}(x)$.

If $2 \notin \phi^{*}(x)$, choose a pendant edge $x v$ such that $\phi(v)=1$ and $\phi(x v)>2$, and then reduce $x v$ 's color to 2 to create a lighter coloring $\psi$. If $1 \notin \phi^{*}(x)$, then among $x$ 's three pendant edges, one $(x u)$ will have a color greater than 3 ; reduce its color to 1 , and reassign $u$ color 2 , to create a lighter coloring $\psi$. Assume, instead, that $1,2 \in \phi^{*}(x)$. Then $\phi^{* *}(x)$ only includes $1,2, \phi(s), \phi(x)$, and one other color $k$. Reassign $x$ a color in $\{3,4,5\} \backslash\{k, \phi(s)\}$ to create a lighter coloring $\psi$.

Case 6: $x$ is a hub of degree 3; then $x$ has two adjacent leaves, $u$ and $v$. Suppose $\phi(x s)>2$. If $\phi(\{u, x u, x v, v\}) \neq\{1,2\}$, then sum of the colors on those elements must be at least 7 , so we can reassign the elements $u, x u, x v$, and $v$ the colors $1,2,1$, and 2 , respectively, to create a lighter coloring $\psi$. If $\phi(\{u, x u, x v, v\})=\{1,2\}$ and $\{\phi(x s), \phi(s)\} \neq\{3,4\}$, then $x$ can be reassigned a color in $\{3,4\} \backslash\{\phi(s x), \phi(s)\}$ to create a lighter coloring $\psi$. If $\phi(\{u, x u, x v, v\})=$ $\{1,2\}$ and $\{\phi(x s), \phi(s)\}=\{3,4\}$, then reassign $x$ color 2 , reassign $v$ color 3 , and 
reassign $x u$ a color in $\{3,4\} \backslash \phi(x s)$ to create a new coloring $\psi$. Then $\operatorname{sum}(\psi)-$ $\operatorname{sum}(\phi)=($ change in $x$ 's color $)+($ change in $v$ 's color $)+($ change in $x u$ 's color $)$ $\leq 2-(\Delta+2)+3-2+4-2=3-\Delta \leq 0$; if this difference is less than zero, then $\psi$ is a lighter coloring; otherwise, the difference is zero but there are fewer elements with a color greater than $\Delta+1$ in $\psi$, so it is a sparser coloring.

Assume otherwise: $\phi(x s) \leq 2$. Then an edge $e \in\{x u, x v\}$ must have color at least 3. If $\phi(e)>3$, reassign $e$ color 3 to create a lighter coloring $\psi$. Otherwise, $\phi(e)=3$. Without loss of generality, let $e=x u$. If $\phi(u)>1$, then we can reduce its color to 1 to create a lighter coloring $\psi$. If $\phi(v) \notin\{1,2\}$, then we can reduce its color to one in $\{1,2\} \backslash \phi(x v)$ to create a lighter coloring $\psi$. Assume, instead, that $\phi(u)=1$ and $\phi(v) \in\{1,2\}$. If $\phi(x v) \notin\{1,2\}$ then $\phi(x v) \geq 4$, so we can reassign it a color $k \in\{1,2\} \backslash \phi(x s)$, and reassign $v$ a color in $\{1,2\} \backslash k$ to create a new coloring $\psi$, which is lighter because $\operatorname{sum}(\psi)-\operatorname{sum}(\phi)=($ change in $x v$ 's color $)+($ change in $v$ 's color $) \leq k-4+(3-k)-1=-2$. Assume, instead, that $\phi(x v) \in\{1,2\}$; then $\phi^{*}(x) \backslash \phi(x)=\{1,2,3\}$. If $\phi(s) \neq 4$, reassign $x$ color 4 to create a lighter coloring $\psi$; otherwise, $\phi(s)=4$, so reassign $x$ color 3 and reassign $x u$ color 4 to create a lighter coloring $\psi$.

Case 7: $x$ is a hub of degree 2. Denote its one leaf neighbor $v$. Since $\phi(v) \neq \phi(x v)$, the sum $\phi(v)+\phi(x v)+\phi(x)$ is at least $3+(\Delta+2) \geq \Delta+5$. Reassign $x$ a color $k_{1} \in\{1,2,3\} \backslash\{\phi(s), \phi(x s)\}$, then reassign $x v$ a color $k_{2} \in\{1,2,3\} \backslash\left\{k_{1}, \phi(x s)\right\}$, then reassign $v$ a color from $\{1,2,3\} \backslash\left\{k_{1}, k_{2}\right\}$ to create a coloring $\psi$ which is lighter because $\operatorname{sum}(\psi)-\operatorname{sum}(\phi)=($ change in $x$ 's color $)+($ change in $x v$ 's color $)$ $+($ change in $v$ 's color $) \leq k_{1}-(\Delta+2)+k_{2}-\phi(x v)+\left(6-k_{1}-k_{2}\right)-\phi(v)=4-\Delta-$ $\phi(x v)-\phi(v)<0$.

In every case, either a lighter, sparser, or weaker coloring is found, which contradicts the definition of $\phi$. The assumption must be false, which makes the theorem true. 
Using a similar technique, it is also possible to prove that a caterpillar cannot be $T$-strong. The following conjecture is offered as a possible subject of further study.

Conjecture: No tree is $T$-strong.

\subsection{The Smallest $T$-Strong Graph}

Theorem 20. The grid graph $G=P_{2} \times P_{3}$ is T-strong.

Proof. This graph can just as easily be defined as the cycle $C_{6}$ plus an edge connecting two vertices of distance 3 . Denote the vertices of the cycle $v_{0}, v_{1}$, etc., up to $v_{5}$, and let $v_{0} v_{3}$ be the extra edge. We can see very quickly that $G$ has total chromatic number at least 4 , because $G$ contains the 4 -cycle $v_{0} v_{1} v_{2} v_{3}$ as a subgraph, which cannot be total-3-colored. Then $\chi^{T}(G)=4$, because a total-4-coloring of $G$ is possible, as shown on the left in Figure 4.2. From this coloring it is implied that the total independence number is at least 4 , because four elements have color 1 . It is impossible for $G$ to have five elements of the same color, because that would imply three of those elements lie on one of the two 4-cycle subgraphs of $G$, and this would contradict $\alpha^{T}\left(C_{4}\right)=2$. Therefore, $\alpha^{T}(G)=4$.

Define $\psi$ to be a total coloring with minimum sum among all total-4colorings of $G$. Then $\psi$ must satisfy 3.1.

Observe that every total independent set $T$ that includes an element in $\left\{v_{0}, v_{3}, v_{0} v_{3}\right\}$ must have size 3 or less: If $T$ includes $v_{0} v_{3}$, then in addition to that, it can only include up to one element from $\left\{v_{1}, v_{2}, v_{1} v_{2}\right\}$ and up to one element from $\left\{v_{4}, v_{5}, v_{4} v_{5}\right\}$, so $|T| \leq 3$. If $T$ includes $v_{0}$, then it can only include up to one other element from $\left\{v_{1} v_{2}, v_{2}, v_{2} v_{3}\right\}$ and one from $\left\{v_{3} v_{4}, v_{4}, v_{4} v_{5}\right\}$, so $|T| \leq 3$. A symmetric argument shows that if $T$ includes $v_{3}$, then $|T| \leq 3$.

From this observation, we can conclude $\psi_{2}<4$. Otherwise, both the first 
and second color classes in $\psi$ have size 4, and therefore cannot include elements from $\left\{v_{0}, v_{3}, v_{0} v_{3}\right\}$. So each of these elements has a color greater than 2. Their mutual proximity implies that they all must have different colors in $\psi$, so one of them must have color 5. But this contradicts the selection of $\psi$.

The restriction $\psi_{2} \leq 3$ implies $\psi_{n} \leq 3$ for all $n>2$, so:

$$
\begin{aligned}
\operatorname{sum}(\psi) & =\sum_{n} n \psi_{n} \\
& \geq(1) 4+\sum_{n \geq 2} n \phi_{n} \\
& \geq 4+(2) 3+\sum_{n \geq 3} n \phi_{n} \\
& \geq 4+6+(3) 3+4 \sum_{n \geq 4} \phi_{n} \\
& \geq 4+6+9+4(3) \\
& \geq 31 .
\end{aligned}
$$
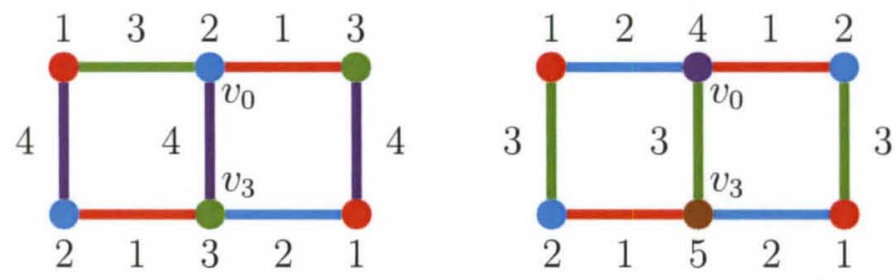

FIGURE 4.2: The grid graph $P_{2} \times P_{3}$ total-colored in two ways.

As $\psi$ was minimum among all total-4-colorings, every total coloring of $G$ that uses only 4 colors must have sum at least 31 . However, $\Sigma^{T}(G) \leq 30$, because a total-5-coloring with sum 30 is possible, as shown on the right in Figure 4.2. As we have seen, this sum is impossible to obtain using only 4 colors, so every optimal total coloring $\phi$ must have $\sigma(\phi)>4$. Thus the total strength of $G, \sigma^{T}(G)$, is at least 5. This is more than its total chromatic number, therefore, $G$ is $T$-strong. 
An exhaustive yet brief analysis of the graphs on five or fewer vertices shows that there is no $T$-strong graph with order less than six. In fact, $P_{2} \times P_{3}$ is the only $T$-strong graph on fewer than nine vertices. This was confirmed by a $\mathrm{C}++$ program that was written to scan all possible graphs and to look at every possible total coloring of each graph, to obtain the total chromatic number, the total chromatic sum, and whether or not an optimal coloring exists with the minimum number of colors.

It seems appropriate to check larger grid graphs to see if they are $T$-strong as well, and this is a good subject for further study. However, every grid graph of the form $P_{2} \times P_{n}$ with $n \geq 4$ can be shown to have an optimal total- $\chi^{T}$-coloring, and is therefore not $T$-strong. (The only known proof for this is lengthy, and the result is not very significant, so it is omitted.)

\subsection{Infinite Families of $T$-Strong Graphs}

For a graph $G$, let an element be called a minority of $G$ if it cannot be included in a maximum total independent set of $G$. Let a set of elements $D$ be called dependent in $G$ if every total independent set in $G$ includes at most one element that is in $D$. An example of a dependent set might be a clique of vertices, or an edge and its endvertices, or all the edges incident to some vertex.

Theorem 21. Let $G$ be a graph with total chromatic number $\chi^{T}$ and total independence number $\alpha^{T}$. Then $G$ is $T$-strong if there is an integer $k>0$ such that:

(1) $G$ contains a dependent set $M$ of $\left(\chi^{T}-k+1\right)$ minorities, and

(2) $\phi_{k}<\alpha^{T}$ implies $\phi$ is not an optimal coloring of $G$.

Proof. Let $k>0$ be an integer such that conditions (1) and (2) are satisfied for a graph $G$. Suppose $\phi$ is an optimal coloring of $G$. Then (2) implies that $\phi_{k}=\alpha^{T}$. The first $k$ color classes are therefore total independent sets of maximum size, so 
no minority can be in one of these color classes. Therefore, every minority has a color greater than $k$. By (1), $M$ is a dependent set of minorities, so every element in $M$ has a different color greater than $k$; this guarantees that $M$ includes some element with color $(k+1)+|M|-1=k+\chi^{T}-k+1=\chi^{T}+1$. Then $\sigma(\phi)>\chi^{T}$. As $\phi$ was an arbitrary optimal coloring, this is true for all optimal colorings, therefore, $\sigma^{T}(G)>\chi^{T}(G)$, so $G$ is $T$-strong.

Several infinite families of $T$-strong graphs exist, which, like $P_{2} \times P_{3}$, consist of a cycle plus one or more chords.

Theorem 22. For each $n, p \in \mathbb{N}$, if $C_{3 n}$ contains a pair of vertices $u$ and $v$ such that dist $C_{3 n}(u, v)=3 p$, then $C_{3 n}+u v$ is $T$-strong.

Proof. Let $n, p \in \mathbb{N}$. The greatest distance between any pair of vertices in $C_{3 n}$ is $\left\lfloor\frac{3 n}{2}\right\rfloor$, so if $3 p$ is greater than this, no pair of vertices exists in $C_{3 n}$ with distance $3 p$, and the theorem is satisfied by default. Assume that $3 p \leq\left\lfloor\frac{3 n}{2}\right\rfloor$; then $p \leq n / 2$.

Label the vertices of a 3 -cycle $C_{3 n}$ as follows: $V=\left\{v_{0}, v_{1}, \ldots, v_{3 n-1}\right\}$ and $E=\left\{v_{0} v_{1}, v_{1} v_{2}, \ldots, v_{3 n-2} v_{3 n-1}, v_{3 n-1} v_{0}\right\}$. Choose a pair of vertices with distance $3 p$; without loss of generality, we can say that one of these is $v_{0}$, and the other is $v_{3 p}$. Let $G=C_{3 n}+v_{0} v_{3 p}$.

Create the total coloring $\phi$ of $G$ in this way: Give the subgraph $G-v_{0} v_{3 p}$ a total-3-coloring by giving every third element in the list $\left(v_{0}, v_{0} v_{1}, v_{1}, v_{1} v_{2}, \ldots\right)$ a color from 1, 2, or 3. Let $\phi\left(v_{0} v_{3 p}\right)=4$. Permute the colors in $\phi$ so that $\phi\left(v_{0}\right)=$ $\phi\left(v_{3 p}\right)=3$, then reassign $v_{0}$ color 5 . The cycle includes $2(3 n)=6 n$ elements, so $\operatorname{sum}(\phi)=(1+2+3)(6 n / 3)+4+(5-3)=12 n+6 ;$ therefore the total chromatic sum $\Sigma^{T}(G)$ must be at most $12 n+6$. Color 1 can be used on $6 n / 3=2 n$ elements in this way, so the total independence number $\alpha^{T}(G)$ must be at least $2 n$.

Note that $\chi^{T}(G) \geq \Delta(G)+1=4$. A total-4-coloring $\pi$ of $G$ exists, which implies $\chi^{T}(G)=4$ : Give the elements $v_{0}, v_{0} v_{1}, v_{1}$, and $v_{1} v_{2}$ the colors $1,3,2$, and 
4, respectively. Then, continue along that path, giving elements the colors 1,2 , 3 , repeating, until finally $v_{3 p}$ is given color 3 . Give the elements $v_{3 p} v_{3 p+1}, v_{3 p+1}$, and $v_{3 p+1} v_{3 p+2}$ the colors 1,2 , and 4 , respectively. Then, continue along that path, giving elements the colors $3,2,1$, repeating, until finally $v_{3 n-1}$ is given color 2 . Finish this by assigning $\pi\left(v_{0} v_{3 p}\right)=4$.

Suppose $T$ is a total independent set in $G$ such that $v_{0} v_{3 p} \in T$. Then the only other elements $T$ can include are those along the paths $v_{1} v_{2} \ldots v_{3 p-1}$ and $v_{3 p+1} v_{3 p+2} \ldots v_{3 n-1}$. The former is a path on $3 p-1$ vertices, which can contain at most $\left\lceil\frac{2(3 p-1)-1}{3}\right\rceil=\left\lceil\frac{6 p-3}{3}\right\rceil=2 p-1$ independent elements, by Theorem 2. The latter is a path on $3 n-3 p-1$ vertices, which can contain at most $\left\lceil\frac{2(3 n-3 p-1)-1}{3}\right\rceil$ $=\left\lceil\frac{6 n-6 p-3}{3}\right\rceil=2 n-2 p-1$ independent elements. Therefore, $T$ contains at most $1+(2 p-1)+(2 n-2 p-1)=2 n-1$ elements, so it does not have maximum size; this implies $v_{0} v_{3 p}$ is a minority.

Suppose $T$ is a total independent set in $G$ such that $v_{0} \in T$. Then $T$ can contain either $v_{3 p-1} v_{3 p}, v_{3 p} v_{3 p+1}$, or neither. In each case, $T$ can contain at most $2 n-1$ elements:

Case 1: $v_{3 p-1} v_{3 p}, v_{3 p} v_{3 p+1} \notin T$. Besides $v_{0}$, the only other elements $T$ can include are those along the paths $v_{1} v_{2} \ldots v_{3 p-1}$ and $v_{3 p+1} v_{3 p+2} \ldots v_{3 n-1}$, although neither $v_{1}$ nor $v_{3 n-1}$ may be in $T$. The former is a path on $3 p-1=2(\bmod 3)$ vertices, which can include at most $2 p-1$ independent non- $v_{0}$ elements, by Theorem 2 . The latter is a path on $3 n-3 p-1=2(\bmod 3)$ vertices, which can include at most $2 n-2 p-1$ non- $v_{3 n-1}$ elements. Therefore, $|T| \leq 1+(2 p-1)+(2 n-2 p-1)=2 n-1$.

Case 2: $\quad v_{3 p-1} \in T$ and $v_{3 p+1} \notin T$. Besides this edge and $v_{0}$, the only other elements $T$ can include are those along the paths $v_{1} v_{2} \ldots v_{3 p-2}$ and $v_{3 p+1} v_{3 p+2} \ldots v_{3 n-1}$, although neither $v_{1}$ nor $v_{3 n-1}$ may be in $T$. The former is a path on $3 p-2=1(\bmod$ 3) vertices, which can include at most $\left\lceil\frac{2(3 p-2)-1}{3}\right\rceil=\left\lceil\frac{6 p-5}{3}\right\rceil=2 p-1$ independent 
elements - but it can only include that many if it includes $v_{1}$, by Theorem 2 , and this is impossible. Therefore, it can include at most $2 p-2$ independent elements. The latter is a path on $3 n-3 p-1=2(\bmod 3)$ vertices, which can contain at most $2 n-2 p-1$ non- $v_{3 n-1}$ elements. Therefore, $|T| \leq 2+(2 p-2)+(2 n-2 p-1)=2 n-1$.

Case 3: $v_{3 p-1} \notin T$ and $v_{3 p+1} \in T$. By the same argument used in case $2,|T| \leq 2 n-1$.

Since $v_{0} \in T$ implies $|T|<\alpha^{T}(G)$ in every case, $v_{0}$ must be a minority. The vertex $v_{3 p}$ is indistinguishable from $v_{0}$ in $G$, so it is a minority as well.

Define $k=2$; then $M=\left\{v_{0}, v_{3 p}, v_{0} v_{3 p}\right\}$ is a dependent set of minorities, where $|M|=3=\chi^{T}-k+1$. Let $\psi$ be a total coloring of $G$ having minimum sum among all total colorings with $\psi_{k}<\alpha^{T}$. Then $\psi_{2}<\alpha^{T}$ implies $\psi$ is not optimal:

$$
\begin{aligned}
\operatorname{sum}(\psi) & =\psi_{1}+2 \psi_{2}+3 \psi_{3}+\sum_{i \geq 4} i \psi_{i} \\
& \geq \psi_{1}+2 \psi_{2}+3 \psi_{3}+4\left(|V|+|E|-\psi_{1}-\psi_{2}-\psi_{3}\right) \\
& \geq-3 \psi_{1}-2 \psi_{2}-\psi_{3}+4(6 n+1) \\
& \geq-3 \alpha^{T}-2\left(\alpha^{T}-1\right)-\left(\alpha^{T}-1\right)+4(6 n+1) \\
& \geq-6(2 n)+3+24 n+4 \\
& \geq 12 n+7 \\
& >\Sigma^{T}(G) .
\end{aligned}
$$

Therefore, by Theorem 21 (using $k=2$ ), $G$ is $T$-strong.

Similar arguments will show that the following graphs, each written below as a cycle plus two chords, are $T$-strong. In every case, $p, q, r \in \mathbb{N}, p+q+r<n$, and $C_{3 n}=v_{0} v_{1} \ldots v_{3 n-1} v_{0}$.

- $C_{3 n}+v_{0} v_{3 p}+v_{3 p+3 q} v_{3 p+3 q+3 r-1}$

- $C_{3 n}+v_{0} v_{3 p}+v_{3 p+3 q-1} v_{3 p+3 q+3 r-2}$ 
- $C_{3 n}+v_{0} v_{3 p}+v_{3 p+3 q-2} v_{3 p+3 q+3 r-1}$

- $C_{3 n}+v_{0} v_{3 p}+v_{3 p+3 q-1} v_{3 p+3 q+3 r}$

An example of a graph from each of these families is shown in Figure 4.3. Every graph in each family has total chromatic number 4 , but requires five colors for an optimal coloring.
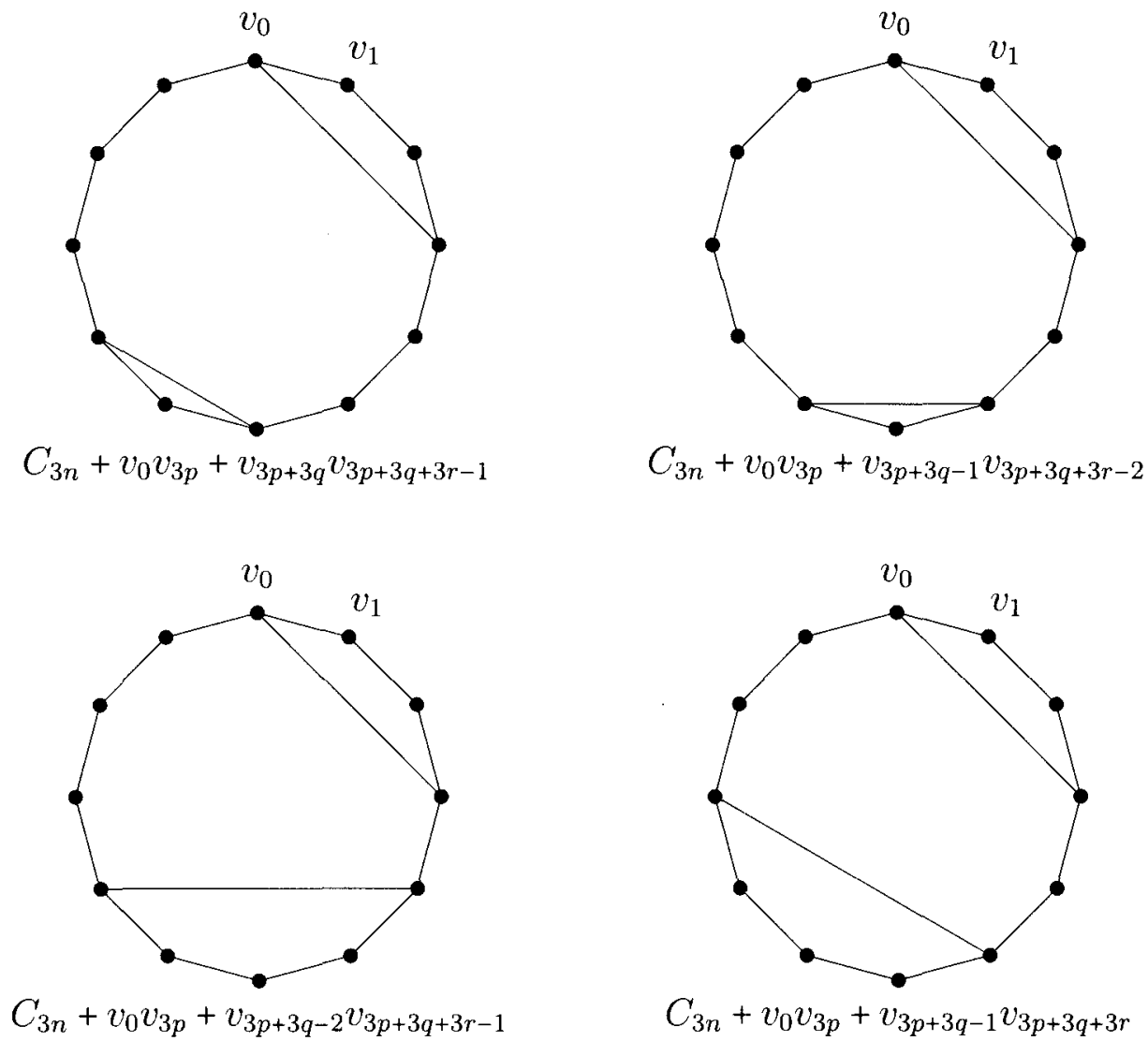

FIGURE 4.3: Four T-strong graphs. 


\section{CONCLUSIONS AND FUTURE WORK}

I believe that the Total Coloring Conjecture is satisfied by planar graphs, and that one day the $\Delta=6$ problem will be solved. However, my search for reducible configurations convinced me that using the same technique will not be enough to do this without using a new technique to prove that larger configurations are reducible. While I am highly motivated to solve this problem, I expect it will remain unsolved for some time.

While the total independence number is important in optimizing total chromatic sums, I think its relationship with the edge domination number makes further exploration of the parameter somewhat redundant. However, it may be worth the time to explore the lower total independence number (the size of the smallest maximal total independent set) and to see if it has any connection to other parameters. After reading Forcade's work, I have developed a peripheral interest in the minimum maximal matching problem, and one of my first future projects will be to work on finding this parameter for hypercubes of dimension higher than six.

As if the determination of $\chi^{T}$ and $\alpha^{T}$ weren't already hard enough - not to mention abstract - the optimization of a total coloring's sum is a fascinating

puzzle. So, too, is the question of which graphs require more colors than necessary to get an optimal sum. When and if I continue down this road, I would like to find more sufficiency conditions for optimality, more sufficiency conditions for $T$ strength, and other graphs that satisfy the property mentioned in Theorem 18. I'd also like to know if there exist graphs for which $\sigma^{T}-\chi^{T}>1$, or if this can be made arbitrarily large. 


\section{REFERENCES}

[1] Robert B. Allan and Renu Laskar, On domination and independent domination numbers of a graph, Discrete Math. 23 (1978), no. 2, 73-76. MR 523402 (80c:05086)

[2] Kenneth Appel and Wolfgang Haken, The solution of the four-color-map problem, Sci. Amer. 237 (1977), no. 4, 108-121, 152. MR 0543796 (58 \#27598e)

[3] Mehdi Behzad, Graphs and their chromatic numbers, Ph.D. thesis, Michigan State University, 1965, p. 69. MR 2615420

[4] O. V. Borodin, On the total coloring of planar graphs, J. Reine Angew. Math. 394 (1989), 180-185. MR 977440 (89m:05049)

[5] Paul Erdős, Ewa Kubicka, and Allen J. Schwenk, Graphs that require many colors to achieve their chromatic sum, Proceedings of the Twentieth Southeastern Conference on Combinatorics, Graph 'Theory, and Computing (Boca Raton, FL, 1989), vol. 71, 1990, pp. 17-28. MR 1041612 (91b:05098)

[6] Rodney Forcade, Smallest maximal matchings in the graph of the $d$ dimensional cube, J. Combinatorial Theory Ser. B 14 (1973), 153-156. MR $0321804(48 \# 171)$

[7] Krzysztof Giaro and Marek Kubale, Edge-chromatic sum of trees and bounded cyclicity graphs, Inform. Process. Lett. 75 (2000), no. 1-2, 65-69. MR 1774835 (2001g:68041) 
[8] Ivan Havel and Mirko Křivánek, On maximal matchings in $Q_{6}$ and a conjecture of R. Forcade, Comment. Math. Univ. Carolin. 23 (1982), no. 1, 123-136. MR 653356 (83h:05069)

[9] Pavol Hell and Donald J. Miller, Graph with given achromatic number, Discrete Math. 16 (1976), no. 3, 195-207. MR 0441778 (56 \#173)

[10] J. E. Hopcroft and M. S. Krishnamoorthy, On the harmonious coloring of graphs, SIAM J. Algebraic Discrete Methods 4 (1983), no. 3, 306-311. MR 711339 (85f:05054)

[11] Tommy R. Jensen and Bjarne Toft, Graph coloring problems, WileyInterscience Series in Discrete Mathematics and Optimization, John Wiley \& Sons Inc., New York, 1995, A Wiley-Interscience Publication. MR 1304254 (95h:05067)

[12] Tao Jiang and Douglas B. West, Coloring of trees with minimum sum of colors, J. Graph Theory 32 (1999), 354-358.

[13] A. V. Kostochka, The total coloring of a multigraph with maximal degree 4, Discrete Math. 17 (1977), no. 2, 161-163. MR 0453576 (56 \#11838)

[14]__ The total chromatic number of any multigraph with maximum degree five is at most seven, Discrete Math. 162 (1996), no. 1-3, 199-214. MR 1425788 (97j:05026)

[15] Ewa Maria Kubicka, The chromatic sum and efficient tree algorithms, Ph.D. thesis, Western Michigan University, 1989, p. 149. MR 2637573

[16] Maxfield Leidner, A larger family of planar graphs that satisfy the total coloring conjecture, Graphs and Combinatorics (2013), to appear. 
[17] Walter Meyer, Equitable coloring, Amer. Math. Monthly 80 (1973), 920-922. MR 0337678 (49 \#2447)

[18] S. Mitchell and S. Hedetniemi, Edge domination in trees, Proceedings of the Eighth Southeastern Conference on Combinatorics, Graph Theory and Computing (Louisiana State Univ., Baton Rouge, La., 1977) (Winnipeg, Man.), Utilitas Math., 1977, pp. 489-509. Congressus Numerantium, No. XIX. MR 0495160 (58 \#13894)

[19] John Mitchem, Patrick Morriss, and Edward Schmeichel, On the cost chromatic number of outerplanar, planar, and line graphs, Discuss. Math. Graph Theory 17 (1997), no. 2, 229-241. MR 1627939 (99i:05086)

[20] Doost Ali Mojdeh and Razieh Sadeghi, Independent edge dominating set of certain graphs, Int. Math. Forum 2 (2007), no. 5-8, 315-320. MR 2296843 (2007k:05160)

[21] Radoš Radoičić and Géza Tóth, The discharging method in combinatorial geometry and the Pach-Sharir conjecture, Surveys on discrete and computational geometry, Contemp. Math., vol. 453, Amer. Math. Soc., Providence, RI, 2008, pp. 319-342. MR 2405688 (2009h:52041)

[22] M. Rosenfeld, On the total coloring of certain graphs, Israel J. Math. 9 (1971), 396-402. MR 0278995 (43 \#4721)

[23] Daniel P. Sanders and Yue Zhao, On total 9-coloring planar graphs of maximum degree seven, J. Graph Theory 31 (1999), no. 1, 67-73. MR 1684286 (2000m:05097)

[24] Lan Shen and Yingqian Wang, On the 7 total colorability of planar graphs with maximum degree 6 and without 4-cycles, Graphs Combin. 25 (2009), no. 3, 401-407. MR 2534896 (2010m:05125) 
[25] Xiang-Yong Sun, Jian-Liang Wu, Yu-Wen Wu, and Jian-Feng Hou, Total colorings of planar graphs without adjacent triangles, Discrete Math. 309 (2009), no. 1, 202-206. MR 2475012 (2010a:05085)

[26] V. G. Vizing, On an estimate of the chromatic class of a p-graph, Diskret. Analiz No. 3 (1964), 25-30. MR 0180505 (31 \#4740)

[27] — The chromatic class of a multigraph, Kibernetika (Kiev) 1965 (1965), no. 3, 29-39. MR 0189915 (32 \#7333)

[28] M. Yannakakis and F. Gavril, Edge dominating sets in graphs, SIAM J. Appl. Math. 38 (1980), no. 3, 364-372. MR 579424 (83c:68086)

[29] H. P. Yap, Total colourings of graphs, Lecture Notes in Mathematics, vol. 1623, Springer-Verlag, Berlin, 1996. MR 1441078 (98c:05071)

[30] Zhongfu Zhang, Jianxun Zhang, and Jingwen Li, On the relations between the parameters of graphs, Taiwanese Journal of Mathematics 10 (2006), no. 6, 1549-1556. 


\section{CURRICULUM VITAE}

Maxfield Edwin Leidner

maxfield.leidner@gmail.com
411 N Taggart Ave, Clarksville, IN (502) 314-0123

\section{Education}

UNIVERSITY OF LOUISVILLE, Louisville, KY 2000 - 2012

Ph.D. in Applied $\mathcal{G}$ Industrial Mathematics (Expected December 2012)

MA in Applied Mathematics, 2010

$B S$ in Mathematics with minor in Physics, 2006

BFA in Studio Art (Sculpture), 2006

\section{Professional Experience}

Summer Instructor for the University of Louisville Math Department Courses taught: College Algebra (2007), Contemporary Mathematics (2008 8 2010), Trigonometry (2011), and Calculus I (2012)

Graduate Teaching Assistant for the U. of Louisville Math Department Subjects taught between 2006 and 2012: College algebra, finite mathematics, comtemporary mathematics, and statistics.

Instructor for Louisville Test Prep

Tutored students and prepared high schoolers for all sections of the SAT, 2010

Fellow for the Groundwork Education in Math and Science Program Co-taught math classes at Farnsley Middle School in Louisville, 2009

Math Tutor with Resources for Academic Achievement, 2002 - 2006

Experience with Maple, $\mathrm{C}++$, Java, $\mathrm{AT}_{\mathbf{E}} \mathrm{X}$, and Microsoft Office 


\section{Dissertation}

"A Study of the Total Coloring of Graphs"

Director: Dr. Grzegorz Kubicki

Committee Members: Dr. André Kézdy, Dr. Ewa Kubicka,

Dr. David Wildstrom, and Dr. Rammohan Ragade

\section{Presentations}

Temperable Graphs and the Total Chromatic Sum.

2012 Meeting of the Kentucky Section of the MAA,

Bellarmine University, Louisville, $K Y$

An Introduction to the Discharging Method.

Mathematics GSO seminar, University of Louisville, Spring 2010

Use of the Discharging Method to Solve a Problem in Total Coloring.

Algebra and Combinatorics Seminar, University of Louisville, Spring 2010

BFA Thesis Exhibition.

University of Louisville Hite Gallery, Spring 2006

\section{Publications}

"A Larger Family of Graphs that Satisfy the Total Coloring Conjecture."

Graphs and Combinatorics (to appear in 2013)

\section{Awards and Community Service}

Judge in the Comp. Sci. section of the Manual High School Science Fair, 2012

Volunteer of Kosair Charity Center in Louisville, Kentucky, 2009

Ken S. and Sandra Hohman Graduate Fellowship in Mathematics, Spring 2007

Resources for Academic Achievement Level II Tutor Certificate, Fall 2006

University of Louisville Trustee's Scholarship, 2000-2004 